\title{
Cutkosky rules and unitarity (violation) in D-instanton amplitudes
}

\author{
Ashoke Sen ${ }^{1}$ \\ Harish-Chandra Research Institute, HBNI, \\ Chhatnag Road, Jhusi, Allahabad 211019, India \\ E-mail: sen@hri.res.in
}

ABSTRACT: In perturbative amplitudes in quantum field theory and string field theory, Cutkosky rule expresses the anti-hermitian part of a Feynman diagram in terms of sum over all its cut diagrams, and this in turn is used to prove unitarity of the theory. For D-instanton contribution to a string theory amplitude, the cutting rule needed for the proof of unitarity is somewhat different; we need to sum over only those cut diagrams for which all the world-sheet boundaries ending on some particular D-instanton lie on the same side of the cut. By working with the closed string effective action, obtained after integrating out the open string modes, we prove that the D-instanton amplitudes actually satisfy these cutting rules, provided the effective action is real. The violation of unitarity in the closed string sector of two dimensional string theory can be traced to the failure of this reality condition. In the critical superstring theory, multi-instanton and multi antiinstanton amplitudes satisfy the reality condition. Contribution to the amplitudes from the instanton anti-instanton sector satisfies the reality condition if we make a specific choice of integration cycle over the configuration space of string fields, whereas contribution due to the non-BPS D-instantons will need to either vanish or have an overall real normalization in order for it to give real contribution. We use Picard-Lefschetz theory to argue that these conditions are indeed satisfied in superstring theories.

Keywords: D-branes, String Field Theory

ArXiv ePrint: 2012.00041

\footnotetext{
${ }^{1}$ Infosys Chair Professor.
} 


\section{Contents}

1 Introduction and summary 1

2 Problems with ordinary cutting rules in open closed string field theory $\begin{array}{lr}\text { on the D-instanton } & 8\end{array}$

3 Reality properties of Euclidean and Lorentzian actions $\quad 13$

$\begin{array}{ll}3.1 & \text { Relation between the Euclidean and the Lorentzian actions } \\ 3.2\end{array}$

$\begin{array}{lll}3.2 & \text { Reality properties of string field theory action } & 17\end{array}$

4 One instanton effective action and its reality properties 20

$\begin{array}{lll}4.1 & \text { Effective action } & 20\end{array}$

4.2 Divergences 22

4.3 Gauge invariance 24

4.4 Reality of the effective action 26

$\begin{array}{ll}4.5 & \text { Cutting rules and unitarity } 28\end{array}$

5 Multi-instanton contribution to the effective action and its reality prop$\begin{array}{ll}\text { erties } & 28\end{array}$

5.1 Construction of the effective action ignoring the zero modes 28

5.2 Effect of divergences and open string zero modes 33

5.3 Identical D-instantons 35

$\begin{array}{ll}5.4 \text { Gauge invariance } & 36\end{array}$

5.5 Reality of the effective action 38

5.6 Cutting rules 41

6 One instanton amplitudes in two dimensional string theory 41

6.1 Leading one instanton contribution 41

6.2 Unitarity of one instanton amplitude for real N 43

6.3 Unitarity violation in the matrix model 47

7 Two instanton amplitudes in two dimensional string theory 48

7.1 Leading two instanton contribution to the closed string two point function 49

7.2 Feynman diagram representation of the amplitude 54

$\begin{array}{lll}7.3 & \text { Unitarity restoration via unitary prescription } & 59\end{array}$

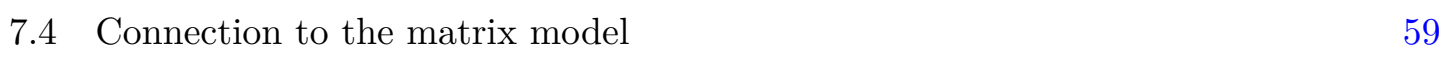

7.5 Three instanton amplitude in the unitary prescription 61

8 Contour prescription from Picard-Lefschetz theory 63

8.1 Non-BPS D-instanton in type IIA string theory 64

8.2 Instanton anti-instanton system in type IIB string theory 66

8.3 Instanton in two dimensional bosonic string theory 67

$\begin{array}{lll}8.4 & \text { Final remarks } & 69\end{array}$

9 Conclusions $\quad 69$ 


\section{Introduction and summary}

Cutkosky rule in quantum field theory expresses the anti-hermitian part of a Feynman diagram in terms of sum of its cut diagrams, and is used to prove perturbative unitarity of the theory [1-5]. In local quantum field theory the Cutkosky rule is proved using a particular property of the position space Green's function known as the largest time equation $[3,4]$. In string (field) theory the interaction vertices in the momentum space representation have exponential dependence on the quadratic functions of momenta, and there is no simple local position space description of these vertices. For this reason the proof based on the largest time equation is not available. Nevertheless it is possible to prove the Cutkosky rules directly by analyzing the momentum space Feynman diagrams of string field theory [6].

The results described above hold for perturbative amplitudes in quantum field theory and string theory to all orders in the coupling constant. However in string theory, certain non-perturbative contributions to the amplitudes may be computed using perturbative techniques. These refer to amplitudes associated to D-instantons [7, 8], and the perturbative technique involves the quantum (field) theory of open and closed strings. Therefore it is natural to ask if one can extend the proof of unitarity of string theory perturbative amplitudes based on Cutkosky rules also to D-instanton induced amplitudes.

Even if string theory is a consistent quantum theory, the answer to this question is not obvious. For example, let us suppose that we consider the scattering of a pair of closed string states at high energy in type IIA string theory. At sufficiently high energy, this process may produce a $\mathrm{D} 0-\overline{\mathrm{D}} 0$ brane pair. Therefore if we consider the scattering amplitudes in the closed string sector alone, where we allow the external states to be only closed strings, we expect the Cutkosky rules to fail. Formally this can be stated by saying that if we integrate out all the degrees of freedom other than closed strings, then the effective action for the closed strings will acquire an imaginary part at energies above the threshold of production of the D0-D̄0 brane pair.

Now suppose that we consider scattering at energies below the threshold of production of the D0- $\overline{\mathrm{D}} 0$ brane pair. Naively one would expect that the unitarity in the closed string sector will be restored, and the closed string effective action below this energy will be real. However, in the open string theory on the D0-D̄0 brane pair, there are classical solutions with energy below this threshold - these are the rolling tachyon solutions $[9$, 10]. The energies of these solutions can go all the way down to zero, and therefore if these are to be counted as independent states in type IIA string theory then we would expect violation of unitarity in the closed string sector even at low energy. In type IIB string theory there are similar low energy configurations associated with rolling tachyons on non-BPS D0-branes to which the closed string states could make transitions. Indeed something similar happens in two dimensional bosonic string theory, where after taking into account the D-instanton corrections to the S-matrix, one finds violation of unitarity in the closed string sector by itself, and this can be traced to possible transition to low energy rolling tachyon configuration on non-BPS D0-branes [11, 12]. There is reason to believe however that in critical string theory the rolling tachyon configurations correspond to some 
configurations of closed string states, and should not be counted as independent states [13]. In particular, if the rolling tachyon system, after quantization, represents new kinds of low mass particles with standard coupling to gravity, they would affect the perturbative amplitudes by appearing as intermediate states in the graviton exchange diagrams, in contradiction to the observation that D-instanton effects are of order $e^{-C / g_{s}}$ for some constant $C$. Therefore we would expect that unitarity is present in the closed string sector itself. Nevertheless it is clearly important to analyze in which theories this happens, and in particular the difference between the two dimensional bosonic string theory and the critical superstring theories that renders the former non-unitary and the latter unitary within the closed string sector.

This is the problem we shall address in this paper. Since the excitations around a D-instanton background involve both open and closed strings, the amplitudes will be expressed as integrals over the moduli spaces of Riemann surfaces with boundaries, with the integrand constructed from the correlation functions of vertex operators on the Riemann surfaces. However even for the usual string amplitudes, this is not an effective approach to proving the cutting rules, since the integrands that appear in these integrals are always real, and the imaginary parts predicted by cutting rules can only arise from the integrals encountering divergences from the boundaries of the moduli spaces, requiring us to define the integrals via analytic continuation, or deforming the integration contour into the complexified moduli space [14-22]. This makes the analysis of the imaginary parts in the world-sheet formalism difficult. For this reason, the natural framework for studying the cutting rules in string theory is string field theory, where we can try to apply the usual method of quantum field theory to try to prove the cutting rules [6]. In the presence of D-instantons, the relevant string field theory is open closed string field theory, describing the dynamics of open and closed strings.

It turns out however, that one cannot directly apply the analysis of [6] to the current problem. One source of the difficulty is that the individual interaction vertices of open closed string field theory in the D-instanton background do not satisfy the usual momentum conservation laws that were used in the analysis of [6]. Another difficulty is that for a given D-instanton system, each amplitude is accompanied by an overall normalization factor independently of the number of vertices and propagators that the Feynman diagram has, and as a result, the cutting rules, that give non-linear relations between the amplitudes, do not hold in their usual form. A third difficulty is that a general cut of a Feynman diagram will have cut running through both open and closed string propagators, but since open strings are not asymptotic states, we should not have cut open string propagators.

We shall circumvent these problems by integrating out the open string modes and defining an effective field theory of closed strings whose interaction vertices satisfy the usual momentum conservation rules. This is somewhat different from the conventional procedure for integrating out a set of fields in a quantum field theory or string field theory [23-26], since here we need to sum over both connected and disconnected diagrams for constructing the interaction vertices of the effective field theory and include the overall normalization factor mentioned in the last paragraph in the definition of the interaction vertices. Nevertheless we find that the action still has the gauge invariance required for 
proving the decoupling of the unphysical states. We can then derive the cutting rules directly in this closed string effective field theory, leading to a proof of unitarity within the closed string sector, provided the action of this effective field theory is real.

In conventional quantum field theory, when we integrate out a set of heavy modes to construct an effective action for the light modes, the effective action is real at low energy, but above the threshold of production of the heavy modes, it acquires an imaginary part, indicating the possibility of transition of the light modes into heavy modes. In contrast, here we are integrating out the open string modes on the D-instanton which are not allowed asymptotic states. Therefore, unitarity requires the effective action to be exactly real. We shall find however that sometime the reality condition may fail to hold. This can arise from various sources that we shall now describe.

1. The boundary state [27-33] describing the D-instanton may be complex, leading to complex contribution to the effective action. The D-instantons in type IIB string theory are of this kind $[8,34]$. However in such cases, one usually finds a complex conjugate boundary state describing the anti-D-instanton, and when one adds the contribution to the effective action from the D-instanton and the anti-D-instanton, one gets real result. In our analysis we shall assume that this is always so, i.e. there is no violation of reality of the effective action from having complex boundary states. In the two dimensional bosonic string theory that we shall discuss in detail, the boundary states of the D-instantons are all real.

2. A D-instanton amplitude is accompanied by an overall normalization factor $\mathbf{N}$ that measures the ratio of the integration measure over the string fields in the D-instanton sector and the vacuum sector, including the ratio of one loop determinants of the string fields in these sectors. If this factor is complex, then the corresponding contribution to the closed string effective action is complex, leading to violation of unitarity. Although it should be possible in principle to determine this constant explicitly by regarding both the D-instanton and the perturbative vacuum as different classical solutions of some string field theory, e.g. the ones involving unstable D-branes, at present we do not have sufficient computational tools to determine this.

In some cases, however, $\mathbf{N}$ may be determined by making use of known duality relations. For example, in two dimensional bosonic string theory, $\mathbf{N}$ can be determined by comparison with the dual matrix model [11] and turns out to be imaginary, causing violation of cutting rules and unitarity in the purely closed string sector of the theory. On the other hand in type IIB string theory, application of S-duality leads to real D-instanton induced term in the effective action [8]. This implies that the constant $\mathbf{N}$ for the D-instanton and the constant $\overline{\mathbf{N}}$ for the anti-D-instanton are complex conjugates of each other, leading to real effective action. In this case the actual phase of $\mathbf{N}$ is ambiguous since it can be absorbed into a shift of the RR scalar field, but we can set $\mathbf{N}$ and $\overline{\mathbf{N}}$ to be real and equal by expanding the action around the vanishing $\mathrm{RR}$ scalar background. 
Since in the presence of a tachyon the steepest descent contour has to run along the imaginary axis, it is reasonable to conjecture that whether $\mathbf{N}$ is real or complex is determined by the absence or presence of tachyons on the D-instanton system. The examples described above are consistent with this.

3. The third source of violation of the reality condition is more directly related to tachyons - those arising from open strings that connect two different D-instantons. If we denote the mass ${ }^{2}$ of this tachyon for coincident D-instantons as $-M^{2}$ and denote by $\chi^{2}$ the squared distance between the D-instantons in the Euclidean space-time, then the mass ${ }^{2}$ of the tachyon in the $\alpha^{\prime}=1$ unit is given by $-M^{2}+\chi^{2} /\left(4 \pi^{2}\right)$. Furthermore these tachyons typically arise in pairs related by the reversal in orientation of the open strings. Then the one loop determinant of these fields is given by a term proportional to:

$$
\left(\chi^{2}-4 \pi^{2} M^{2}\right)^{-1} .
$$

At higher orders, there will also be such terms from internal open string tachyon propagators. Since $\chi$ represents a mode of the open string and needs to be integrated over to define the closed string effective action, we need a prescription for dealing with the singularities at $\chi= \pm 2 \pi M$. The prescription of [12, 35], called the Lorentzian prescription, is to replace (1.1) by:

$$
\left(\chi^{2}-4 \pi^{2} M^{2}+i \epsilon\right)^{-1} .
$$

However, this typically generates imaginary contribution to the closed string effective action and leads to violation of unitarity.

The violation of unitarity associated with the Lorentzian prescription (1.2) was not a problem in the analysis of [12], where the underlying theory is not unitary when restricted to purely closed string sector and the lack of unitarity signals possible transition to rolling tachyon configuration on the D0-brane. However, if we use the same prescription for the instanton anti-instanton sector of type IIB string theory we shall get unitarity violating amplitudes in the closed string sector, which, as we have discussed earlier, does not seem likely. For this reason, we suggest a different prescription, where we deal with the singularities of the type given in (1.1) by averaging over the Lorentzian prescription of [12] and its complex conjugate. This means that we replace all the $i \epsilon$ 's in the Lorentzian prescription by $-i \epsilon$ and then take the average of the original result and the new result. This leads to real closed string effective action, restoring unitarity. We shall call this the unitary prescription. Physically, these two prescriptions can be regarded as different choices of integration cycles for the variables $\chi$. Since the modes $\chi$ are particular modes of the open string field, the choice of integration cycle refers to the choice of integration cycle in the space of string fields. The unitary prescription also seems to be needed in two dimensional type 0B string theory [36].

For a general amplitude with $k$ instantons the singularity structure is quite complicated. For example if we denote by $\phi_{i}$ the Euclidean space-time coordinate of the $i$-th D- 
instanton, then in general the singular factor in the Lorentzian prescription takes the form,

$$
\prod_{\substack{i, j=1 \\ i<j}}^{k}\left\{\left(\phi_{i}-\phi_{j}\right)^{2}-4 \pi^{2} M_{i j}^{2}+i \epsilon\right\}^{-a_{i j}},
$$

for some integers $a_{i j}$. In the unitary prescription one needs to average between (1.3) and its complex conjugate. Despite the proliferation of singularities of the kind given in (1.3) for large number of instantons, one can check that both the Lorentzian and the unitary prescriptions give non-singular results, i.e. the integration contour is not pinched between a pair of poles from the opposite side. For the Lorentzian prescription this follows from the fact that deforming each $\phi_{i}$ integration contour to $\phi_{i}=e^{i \theta} u_{i}$ with real $u_{i}$ and $\theta>0$, we can move away from the singularities, since in the complex $u_{i}$ plane the poles are now at $\left(u_{i}-u_{j}\right)^{2}=\left(4 \pi^{2} M_{i j}^{2}-i \epsilon\right) e^{-2 i \theta}$, i.e. away from the real axis, and furthermore, as we vary $\theta$ from 0 to some positive value, the poles do not cross the real $u_{i}$ axis along which the integration contour lies. For this argument we must use the same $\theta$ for all components of all the $\phi_{i}$ 's, so that the difference between any pair of $\phi_{i}$ 's also has the same phase $e^{i \theta}$. For the complex conjugate contour, needed for the unitary prescription, we can similarly deform the $\phi_{i}$ integration contour to $e^{-i \theta} u_{i}$ with real $u_{i}$ and $\theta>0$ to move away from the singularities. In contrast, if we had used other prescriptions containing product of terms in which some have $i \epsilon$ and others have $-i \epsilon$, the contour could get pinched between poles from opposite sides, since the number of denominator factors given by the number of independent pairs of D-instantons grows faster than the number of integration variables which is proportional to the number of D-instantons. However other special choices that avoid singularities may be possible, and given any such choice, there is another choice in which we change the sign of all the $i \epsilon$ 's. As long as we always take the average of such pair of choices, we shall get unitary theory, provided the normalization constants $\mathbf{N}$ are real.

Since [12] found agreement between the matrix model results and string theory results with the Lorentzian prescription, one might ask if the agreement is spoiled when we use the unitary prescription. A conservative answer to this question is that since in two dimensional string theory we expect violation of unitarity in the closed string sector anyway, one could continue to use the Lorentzian prescription for this theory and use unitary prescription for the critical string theories. We have nevertheless explored what happens to the agreement with the matrix model result if we use the unitary prescription to define the instanton amplitudes in two dimensional string theory. We find that for the leading two instanton contribution to the $n$ point amplitude of closed strings and the leading 3-instanton contribution to the 2-point amplitude of closed strings, the difference between the two prescriptions can be compensated for by a change in the normalization constants $\mathbf{N}$ for $(2,1)$ and $(3,1)$ ZZ-instantons [37]. For example in the analysis of [12], the normalization constants (in our notation) for the $(2,1)$ and $(3,1)$ instantons would be given by $\mathbf{N}_{2}=-3 i /\left(64 \pi^{2}\right)$ and $\mathbf{N}_{3}=5 i /\left(192 \pi^{2}\right)$ respectively. In contrast in the unitary prescription the agreement with the matrix model results determines these constants to be $-i /\left(32 \pi^{2}\right)$ and $i /\left(96 \pi^{2}\right)$ respectively. ${ }^{1}$ Eventually it may be possible to explain these

\footnotetext{
${ }^{1}$ Similar results were observed independently by Balthazar, Rodriguez and Yin [36].
} 
results as a result of contour deformation in the configuration space of string fields, but this will require a better understanding of this configuration space. Note that in the unitary prescription, the unitarity violation in the two dimensional string theory can be attributed solely to the imaginary values of the normalization constants $\mathbf{N}$.

Based on all the observations described above, we can summarize the status of our understanding of D-instanton amplitudes in the critical superstring theory as follows:

1. We have argued that for BPS instantons the normalization constant $\mathbf{N}$ for the instanton and the anti-instanton are complex conjugates of each other. Even though this is determined by examining a particular term in the effective action that is protected by supersymmetry, since the normalization constant is the same for all amplitudes, we conclude that the closed string effective action associated with the single instanton and single anti-instanton amplitudes are real. Furthermore, since the normalization constants for multi-instanton amplitudes are determined from that of single instanton amplitudes, and since no tachyon appears on the multi-instanton or multi-antiinstanton sectors anywhere in the moduli space of instantons, we can conclude that the contribution to the closed string effective action from the multi-instanton and multi-anti-instanton sectors are also real. Therefore there is no violation of unitarity in the closed string sectors from these amplitudes.

2. The situation is different for amplitudes in the instanton anti-instanton sector and for non-BPS D-instantons that carry tachyonic modes on their world-volume. In the former case we can have real closed string effective action and therefore unitary amplitudes if we choose the unitary prescription, but there are other choices of integration contour that leads to complex effective action. In the presence of nonBPS D-instantons, the steepest descent contour runs along the imaginary axis for the tachyon field, suggesting that the normalization constant $\mathbf{N}$ should be imaginary. This would suggest imaginary contribution to the closed string effective action unless $\mathbf{N}$ vanishes.

While we cannot fully resolve the ambiguities mentioned above, we have used PicardLefschetz theory and general properties of the tachyon potential on non-BPS D-instanton and instanton - anti-instanton system, to argue that for the former case $\mathbf{N}$ can indeed be taken to vanish, and in the latter case unitary prescription is the appropriate prescription for computing the effective action. These arguments depend on a particular natural choice of integration contour for the tachyonic modes that keeps the tachyons real along the contour. On the other hand, for two dimensional bosonic string theory, this natural choice of contour does not exist, forcing the theory to be non-unitary. It should be noted however that even in critical superstring theories, other choices of integration contour that violate unitarity are also possible.

We shall now summarize the rest of the paper. In section 2 we describe why the usual cutting rules, needed for the proof of unitarity, must be modified in the open closed string field theory $[38,39]$ on D-instantons. In section 3 we discuss some aspects of the reality properties of string field theory action. In section 4 we describe how we can prove 
the modified cutting rules, required for unitarity, for amplitudes induced by a single Dinstanton, provided we assume certain reality property of the overall normalization constant that multiplies a D-instanton amplitude. This normalization constant cannot be computed with the currently available techniques, but there is indirect evidence that the required reality properties may fail if the D-instanton system has open string tachyonic modes. In section 5 we extend the result to multi-instanton amplitudes, where we find a more direct relation between the existence of open string tachyonic modes and the reality properties of the closed string effective action. We discuss the Lorentzian and unitary prescriptions for integrating over the open string zero modes and show how using the unitary prescription one can avoid the breakdown of reality properties of the effective action, provided the overall normalization constants associated with single instanton amplitudes are real. In section 6 we illustrate the cutting rules for single D-instanton amplitudes with a specific example in two dimensional string theory [11, 12, 40-47]. We demonstrate that the cutting rules hold when the overall normalization constant satisfies the required reality properties, and the breakdown of unitarity found in [11] can be traced solely to the imaginary value of the normalization constant. In section 7 we analyze the unitarity of 2-instanton amplitudes in two dimensional bosonic string theory. We demonstrate that even if we choose the normalization constant appearing in the one instanton sector in a way that gives unitary amplitude, the cutting rules still fail in the two instanton sector if we use the Lorentzian contour prescription of [12] to integrate over the open string zero modes. This failure can be traced directly to the appearance of a tachyonic mode of the open string connecting the two D-instantons below a critical separation. However we also show that unitarity is restored if we use the unitary prescription for the integration contour, provided the overall normalization constants are chosen to be real. In section 8 we use Picard-Lefschetz theory to argue that in type IIA and IIB superstring theories, where the tachyon potential is bounded from below, we have natural choice of integration contours for which there is no violation of unitarity in the closed string sector due to D-instantons.

We shall end this introduction with some final remarks. Since the amplitudes in critical string theory are not Borel summable, a critical reader may want to take the point of view that one cannot trust the instanton contribution to these amplitudes before we have understood the perturbative corrections fully. While this is a valid criticism for general string amplitudes, not every quantity that one can compute in perturbation theory has this problem. In particular, the matrix elements of $S\left(g_{s}^{*}\right)^{\dagger} S\left(g_{s}\right)$ between closed string states is known to be given by those of the identity matrix to all orders in perturbation theory due to perturbative unitarity of superstring theories. Therefore it makes sense to compute the D-instanton corrections to these quantities. Any non-zero contribution to these matrix elements will signal violation of unitarity within the closed string sector and possibly point to the existence of new sectors that must be included in the theory. Another motivation comes from the study of resurgence [48-51] which shows that formal power series expansions may be useful in extracting non-perturbative information on the theory even when the perturbation series is not Borel summable. Understanding the systematic power series expansion of the instanton contribution to the amplitude will be an additional input to such formal power series expansions, besides the usual perturbation expansion. 


\section{Problems with ordinary cutting rules in open closed string field theory on the D-instanton}

Let $S=1+i T$ be the S-matrix of a theory. Then the unitarity relation $S^{\dagger} S=1$ may be expressed as

$$
\langle f|T| i\rangle-\left\langle f\left|T^{\dagger}\right| i\right\rangle=i\left\langle f\left|T^{\dagger} T\right| i\right\rangle=i \sum_{n}\left\langle f\left|T^{\dagger}\right| n\right\rangle\langle n|T| i\rangle,
$$

where $|i\rangle$ and $\langle f|$ are the incoming and outgoing states respectively, and the sum over $n$ on the right hand side runs over a complete set of states in the theory. As in [6], throughout this paper we shall refer to the matrix elements of $T$ as amplitudes, without any extra normalization factor. However, one small difference with [6] is that there we defined $T$ through $S=1-i T$ whereas here we have defined it via $S=1+i T$. This is responsible for the opposite sign on the left hand side of (2.1) compared to [6].

For perturbative amplitudes the right hand side of (2.1) is interpreted as sum over cut diagrams, in which we draw a cut through the diagram dividing it into two parts, with all the incoming lines on the left side of the cut and all the outgoing lines on the right side of the cut. The rules for evaluating the contribution from a cut diagram require hermitian conjugating the part of the diagram to the right of the cut, ${ }^{2}$ and replacing the $i /\left(-\ell^{2}-m^{2}+i \epsilon\right)$ factor of each internal cut propagator by $2 \pi \delta\left(\ell^{2}+m^{2}\right) \Theta\left(\ell^{0}\right)$ where $\ell$ is the momentum carried by the propagator from left to the right and $m$ is the mass of the state propagating along the cut line. A cut on an external line has no effect and a cut does not pass through any interaction vertex. In that case the collection of the cut lines represent the state $|n\rangle$, the left side of the diagram containing the original incoming states and outgoing cut lines represent $\langle n|T| i\rangle$ and the right side of the diagram containing the original outgoing states and incoming cut lines represent $\left\langle f\left|T^{\dagger}\right| n\right\rangle$. Therefore cutting rules, relating the difference between an amplitude and its hermitian conjugate to the sum of cut diagrams, establish unitarity of the theory. There are some additional subtleties involving sign factors for disconnected diagrams that can be found in [6]. Due to gauge invariance of string field theories, there are additional complications due to possible contribution of unphysical states along the cut propagator, and these have to be analyzed separately [52].

For D-instanton induced amplitudes, the cutting rules required for proving unitarity are somewhat different. To see this, we need to recall a few facts about the D-instanton induced amplitudes:

1. The world sheet description of the amplitudes in the presence of D-instantons involves integration over moduli spaces of punctured Riemann surfaces as usual, but now we also have Riemann surfaces with boundaries, with D-instanton boundary condition at the boundary. This in particular involves imposing Dirichlet boundary condition on the (Euclidean) time coordinate, and other non-compact spatial coordinates.

2. All single D-instanton induced amplitudes are accompanied by a single multiplicative factor of $\mathbf{N} e^{-C / g_{s}}$ where $\mathbf{N}$ is a normalization constant and $C / g_{s}$ is the instanton

\footnotetext{
${ }^{2}$ As in [6], we define the hermitian conjugate of a diagram by reversing the sign of all external momenta and charges and then taking the complex conjugate of the amplitude.
} 
action, irrespective of how many boundaries or how many disconnected components the world-sheet has. Formally $\mathbf{N}$ can be interpreted as the exponential of the annulus diagram with ends lying on the D-instanton. If there are $k$ D-instantons, then the exponential factor is replaced by $e^{-k C / g_{s}}$ and the normalization factor also changes to $\mathbf{N}^{k}{ }^{3}$ The $\mathbf{N}^{k}$ factor accounts for exponential of sum of $k$ annulus diagrams, with the $i$-th annulus having both its boundaries lying on the $i$-th D-instanton. There are also annulus diagrams with the two ends lying on two different D-instantons, but these contributions are finite and depend on the locations of the D-instantons, and must be included separately in the computation of the amplitude.

3. Typically these amplitudes suffer from various infrared problems, which can all be systematically dealt with by representing them as sum of Feynman diagrams in openclosed string field theory [38, 39]. In this formalism, the D-instanton induced amplitudes are built from Feynman diagrams whose interaction vertices are given by correlation functions of open and closed string vertex operators and ghost fields on appropriate Riemann surfaces, integrated over subspace of the moduli spaces of the Riemann surfaces. These subspaces do not include any degenerate Riemann surface, and therefore the interaction vertices are completely regular.

4. In this open-closed string field theory, the open strings carry strictly zero momentum due to Dirichlet boundary condition associated with D-instantons and therefore represent zero dimensional fields, but closed string states are regular fields of the theory and the asymptotic states are built out of the closed strings. Due to vanishing momenta, the path integral over the open string zero modes cannot be treated via Feynman diagrams, since the propagators are infinite. One needs to remove their contribution from the internal propagators of a Feynman diagram, and carry out the path integral over these modes at the end of the calculation [53]. The relevant zero modes, after appropriate field redefinition, consist of a set of zero modes in the ghost sector of string field theory, one for each D-instanton, and the collective modes describing the location of each D-instanton in space-time. The integration over the ghost zero modes may be interpreted as division by the volume of the rigid $\mathrm{U}(1)$ gauge group that exists on each D-instanton, and may be absorbed into the overall normalization constant $\mathbf{N}$ common to all amplitudes. The integration over the collective modes need to be carried out explicitly at the end of the calculation.

5. We shall divide the interaction vertices of this open closed string field theory into two types. If the Riemann surface associated with the interaction vertex does not have a boundary we shall call it an ordinary vertex since it represents the usual vertex of a closed string field theory. On the other hand if the Riemann surface has one or more boundaries on which we impose D-instanton boundary condition, then we shall call this the D-instanton type vertex.

\footnotetext{
${ }^{3}$ If there are different types of D-instantons with different actions, then in the exponent we shall get the sum of the actions of different D-instantons and the normalization constant will be given by the product of the normalization constants for different D-instantons.
} 
6. The ordinary interaction vertices are accompanied by the usual momentum conserving delta function, but the D-instanton type interaction vertices do not have such delta functions, since Dirichlet boundary condition on the non-compact space-time directions break translation invariance.

7. The overall momentum conservation is restored at the end when we integrate over the collective modes of the open string describing the location of the D-instanton in space and time.

Now we are in a position to explain why the ordinary cutting rules are not sufficient to establish the unitarity relation given in (2.1). There are several ways to see this. We shall first consider the case of a single D-instanton.

1. Let $T$ and $T^{\dagger}$ on the left hand side of (2.1) denote one instanton contributions to the amplitudes. Both are proportional to $e^{-C / g_{s}}$. On the other hand, for a generic cut of the original diagram, the diagrams on both sides of the cut will have D-instanton type interaction vertices and will therefore carry $e^{-C / g_{s}}$ factors. Therefore their product will be proportional to $e^{-2 C / g_{s}}$. This will be in clear disagreement with the left hand side of (2.1)

2. Consistency with (2.1) requires that there should be momentum conserving delta function accompanying the individual diagrams on either side of the cut, since they are supposed to represent physical amplitudes encoded in the matrix elements of $T$ and $T^{\dagger}$ appearing on the right hand side of (2.1). On the other hand, if the cut had D-instanton type interaction vertices on both sides, then the original diagram had no such momentum conserving delta function for parts of the diagrams on either side of the cut, although there was an overall momentum conserving delta function for the full diagram. Since the usual cutting rules do not generate an extra momentum conserving delta function in the cut diagram, there is a clear conflict between the usual cutting rules and (2.1).

3. A generic cut of the original diagram will also cut the open string propagators. If we try to represent such a cut diagram by a term on the right hand side of (2.1), then the sum over $|n\rangle$ in (2.1) will have to contain open string states as well as closed string states. This is inconsistent with the fact that open string states on the Dinstanton are not asymptotic states. Therefore they should not appear in the sum over intermediate states in (2.1).

This suggests that we should somehow modify the cutting rules so that instead of summing over all cuts of the original diagram, we sum over only those cuts for which all the D-instanton induced interaction vertices are on one side of the cut. In this case the amplitude on only one side of the cut will have $e^{-C / g_{s}}$ factor and therefore the product of the amplitudes on the two sides of the cut will be proportional to $e^{-C / g_{s}}$ as required. Also, the amplitude on the side of the cut that involves ordinary interaction vertices, will satisfy momentum conservation since each vertex conserves momentum. Therefore the conservation 
of total momentum in the original diagram will also lead to conservation of momentum on the side of the cut that carries D-instanton type vertices. Finally since open strings can only begin and end on D-instanton type vertices, and since all the D-instanton type vertices are on one side of the cut, there are no cut open string propagators, in accordance with the expectation that open strings are not asymptotic states.

Required cutting rules for multi-instanton contribution to the amplitude may be analyzed in a similar way. If there are $k$ instantons then a given boundary of the world-sheet may have $k$ possible boundary conditions - one for each D-instanton. We shall consider the general case where they are different D-instantons, but the discussion also applies to the case of identical D-instantons since they can still be distinguished by their locations in space-time. Let us call the boundary condition for the $i$-th D-instanton the $i$-type boundary. An interaction vertex of the open closed string field theory will now carry a set of labels $\left(i_{1}, \cdots, i_{m}\right)$ if boundaries of type $i_{1}, \cdots, i_{m}$ are present on the associated Riemann surface. Note that a given boundary may have many type of segments since the vertex operator of an open string starting on the $i$-th D-instanton and ending on the $j$-th Dinstanton, inserted on a boundary, connects segments of type $i$ and $j$. All such segments have to be included in the labels $\left(i_{1}, \cdots, i_{m}\right)$ of an interaction vertex. Following the same argument as before, one can argue that the right hand side of (2.1) now should represent a sum over cut diagrams such that all boundaries of a given type are present on one side of the cut. In other words, the left side of the cut will contain a subset $S \subset\{1, \cdots, k\}$ of the boundary types and the right side of the cut will involve the complementary subset $S^{c}$ of the boundary types. Therefore all the interaction vertices on the left of the cut must be of type $\left(i_{1}, \cdots, i_{m}\right)$, with $i_{1}, \cdots, i_{m} \in S$ and all the interaction vertices on the right of the cut must be of type $\left(j_{1}, \cdots, j_{p}\right)$ with $j_{1}, \cdots, j_{p} \in S^{c}$. If $C_{i} / g_{s}$ denotes the action of the $i$-th D-instanton then the weight factors associated with the left and the right sides of the cut, regarded as separate amplitudes as on the right hand side of (2.1), will have the factors $e^{-\sum_{i \in S} C_{i} / g_{s}}$ and $e^{-\sum_{i \in S^{c}} C_{i} / g_{s}}$ respectively, and their product gives $e^{-\sum_{i=1}^{k} C_{i} / g_{s}}$. On the other hand if each side had vertices of all types, then each will have a weight factor $e^{-\sum_{i=1}^{k} C_{i} / g_{s}}$ and their product would have given $e^{-2 \sum_{i=1}^{k} C_{i} / g_{s}}$, in disagreement with the weight factor $e^{-\sum_{i=1}^{k} C_{i} / g_{s}}$ of the original amplitude.

This shows that the desired cutting rules in open closed string field theory on the D-instanton are different from those in ordinary quantum field theories. Let us now review what goes wrong in the proof of the conventional cutting rules in open closed string field theory on the D-instanton. For this we recall the main assumptions of [6] that went behind the proof of the cutting rules for perturbative string amplitudes:

1. The amplitudes are given by the usual sum of Feynman diagrams expressed as integrals over loop momenta.

2. The action from which the Feynman rules are derived is real.

3. The propagators have the usual pole structure, but we do not make any assumption of how many particles the theory has or what their masses are. 
4. The interaction vertices fall off exponentially in the direction of imaginary energy and real spatial momenta. This assumption is not essential for the proof of the cutting rules, but is a property of string field theory that ensures ultra-violet finiteness of the amplitudes.

5. The interaction vertices have the usual momentum conservation laws.

For D-instanton induced amplitudes, one of the assumptions that fails in the proof of cutting rules is momentum conservation at the interaction vertices of open-closed string field theory relevant for computing the amplitudes. As already discussed, this failure is due to the breaking of translational invariance by the D-instanton boundary condition. The overall momentum conservation is restored at the end after integration over a particular collective mode of the open string field, describing the average location of all the D-instantons in space-time. However the individual interaction vertices of open closed string field theory do not satisfy the momentum conservation rule. This in turn prevents us from proving the cutting rules in the open closed string field theory in the usual form. For example, in the analysis of [6], the constraints on internal momenta due to momentum conservation at the interaction vertices were used to determine what kind of contour deformations are allowed in the complex plane of the internal energies. This in turn was used to determine the regions of integration that contribute to the anti-hermitian part of an amplitude, leading to the cutting rules in the form described earlier.

The first assumption, that the amplitudes are given by usual sum over Feynman diagrams, also fails for D-instanton amplitudes. The overall factor of $\mathbf{N} e^{-C / g_{s}}$ accompanying a D-instanton amplitude cannot be assigned to either an interaction vertex or a propagator of the Feynman diagram, since this factor does not depend on the number of propagators and interaction vertices in the diagram. For this reason any non-linear relation between the amplitudes derived from the analysis of Feynman diagrams, like the cutting rules, will fail to hold in its usual form.

There is another issue in open-closed string field theory on D-instantons that was not present in the analysis of the cutting rules in closed string field theory. The reality of the action needed for the proof of the cutting rules is formulated naturally in the Lorentzian theory. However since D-instantons are solutions in the Euclidean theory, the open string fields are defined naturally in the Euclidean space.

We shall see in section 4 and section 5 that these problems can be addressed by integrating out the open string fields to define an effective action of the closed string fields in the Euclidean space and then analytically continuing this action to the Lorentzian space. The interaction vertices of this effective field theory will satisfy the usual momentum conservation laws. Therefore we can now try to apply the cutting rules directly in this closed string effective field theory. The reality of the effective action is not guaranteed however, and we shall see that this can fail when the D-instanton system has open string tachyons. This in turn leads to failure of unitarity of the closed string sector by itself. However when the effective action is real, the usual cutting rules hold in the closed string effective field theory, leading to unitary amplitudes. Furthermore, we can also translate these cutting 
rules to the original open closed string field theory, and show that these cutting rules precisely correspond to the ones that are needed for the unitarity of the theory.

\section{Reality properties of Euclidean and Lorentzian actions}

Since D-instantons are defined as solutions in Euclidean string theory, they are used to first compute corrections to the Euclidean action of string field theory. This then has to be Wick rotated to Lorentzian signature, to check if it satisfies the properties required for the proof of the cutting rules. The main properties that will be relevant are momentum conservation at the interaction vertices and the reality of the action. For this reason we shall begin in section 3.1 by reviewing some standard relations between Euclidean and Lorentzian action. Section 3.2 contains a discussion of string field theory actions and their reality properties.

\subsection{Relation between the Euclidean and the Lorentzian actions}

In this section we shall set up some notations on Wick rotation. Even though this is standard textbook material, we have included this discussion, since various factors of $i$ and their signs will play a crucial role in our analysis.

Let us denote by $x=\left(x^{0}, \cdots x^{D-1}\right)$ the space-time coordinates in the Lorentzian space and by $x_{E}=\left(x_{E}^{0}, \cdots, x_{E}^{D-1}\right)$ the space-time coordinates in the Euclidean space. The conjugate momentum variables will be denoted by $p=\left(p_{0}, \cdots, p_{D-1}\right)$ and $\left(p_{0}^{E}, \cdots, p_{D-1}^{E}\right)$ respectively. ${ }^{4}$ Then the usual analytic continuation rule sets the spatial coordinates / momenta in the two formalisms to be equal and the time coordinates are related as,

$$
x^{0}=-i x_{E}^{0}, \quad p_{0}=i p_{0}^{E},
$$

so that the time evolution operator $e^{-i H x^{0}}$ in the Lorentzian theory is mapped to $e^{-H x_{E}^{0}}$ in the Euclidean theory. Note that in the Lorentzian theory we have used $p_{0}$ as the independent variable since this is conjugate to $x^{0}$. When we refer to $p^{0}$ it should be regarded as $-p_{0}$.

It is instructive to recall how the analytic continuation is done. For this we introduce the interpolating variables:

$$
x_{\theta}^{0}=e^{-i \theta} u, \quad p_{0}^{\theta}=e^{i \theta} v,
$$

with real variables $u$ and $v$. For $\theta=0$ we interpret $u$ as $x^{0}$ and $v$ as $p_{0}$, while for $\theta=\pi / 2$, we interpret $u$ as $x_{E}^{0}$ and $v$ as $p_{E}^{0}$. This can be summarized by saying that in position space we rotate clockwise as we go from the Lorentzian to the Euclidean theory and rotate anti-clockwise as we go from the Euclidean to the Lorentzian theory. On the other hand, in momentum space we rotate anti-clockwise as we go from the Lorentzian to the Euclidean theory and rotate clockwise as we go from the Euclidean to the Lorentzian theory.

If we consider an integral over $x_{\theta}^{0}$ or $p_{0}^{\theta}$ that appear e.g. in a Fourier transformation, then if the integration contour is rotated by changing $\theta$, the combination $x_{\theta}^{0} p_{0}^{\theta}$ that appears

\footnotetext{
${ }^{4}$ We shall use both subscript $E$ and superscript $E$ to denote Euclidean variables.
} 
in the exponent remains real. Since we have:

$$
d x_{\theta}^{0}=e^{-i \theta} d u, \quad d p_{0}^{\theta}=e^{i \theta} d v,
$$

and since under contour rotation the integration measure remains $d x_{\theta}^{0}$ or $d p_{0}^{\theta}$, it follows that under the Wick rotation we shall have,

$$
d x^{0}=-i d x_{E}^{0}, \quad d p_{0}=i d p_{0}^{E} .
$$

On the other hand the relation $p^{0}=-p_{0}$ is simply a change of variable and not a contour rotation. Therefore the integrals do not pick up any sign when we replace $p_{0}$ by $p^{0}$ since the change in measure is compensated for by a change in the integration limits. Since $p^{0}=-p_{0}$ corresponds to energy $\omega$ in the Lorentzian theory, we shall refer to $-p_{0}^{E}$ as the Euclidean energy $\omega_{E}$.

We shall denote by $S_{E}$ and $S$ the Euclidean and the Lorentzian actions, expressed as momentum space integrals with sum of products of fields and momentum factors in the integrand. We shall assume that $S_{E}$ and $S$ have been normalized such that the Euclidean path integral is weighted by $e^{S_{E}}$ and the Lorentzian path integral is weighted by $e^{i S}$. We shall show that with this definition $S$ is obtained from $S_{E}$, with both represented as momentum space integrals, by making the following replacements:

1. We replace in the integrand the Euclidean momentum conserving delta function by the Lorentzian momentum conserving delta function, and integral over the Euclidean momenta $p_{E}$ by integral over Lorentzian momenta $p$.

2. We replace, in the argument of the scalar fields, the Euclidean momenta by Lorentzian momenta i.e. we have

$$
\phi_{E}\left(p_{E}\right) \rightarrow \phi(p)
$$

Note that this is a replacement rule and not an equality. The latter is discussed in (3.9).

3. Inside the argument of any explicit function $f$ of Euclidean momenta multiplying the integrand, we should express the Euclidean momenta in terms of Lorentzian momenta using (3.1). For example a factor of $\left(p_{0}^{E}\right)^{2}$ in $f$ will be replaced by $-\left(p_{0}\right)^{2}$ in the Lorentzian theory.

4. For tensor fields, the replacement rule will have extra factors of $i$ for every 0 component of the fields, e.g.

$$
A_{E}^{0}\left(p_{E}\right) \rightarrow i A^{0}(p)=-i A_{0}(p)
$$

5. For fermion fields the relationship between the Euclidean and the Lorentzian fields is more complicated. In general this will require us to work with complex fields in both, the Euclidean and the Lorentzian theory, since the reality properties of fermions differ in the two cases. Later we shall describe a way to avoid this problem. 
We shall illustrate this by taking the example of a scalar field $\phi$ in the momentum representation. Let us consider a term in $S_{E}$, expressed as a momentum space integral of the form

$$
\begin{aligned}
& \int d^{D} p_{(1)}^{E} \cdots d^{D} p_{(n)}^{E} \delta^{(D)}\left(p_{(1)}^{E}+\cdots+p_{(n)}^{E}\right) \prod_{i=1}^{n} \phi_{E}\left(p_{(i)}^{E}\right) \\
&=\int d^{D} p_{(1)}^{E} \cdots d^{D} p_{(n-1)}^{E}\left\{\prod_{i=1}^{n-1} \phi_{E}\left(p_{(i)}^{E}\right)\right\} \phi_{E}\left(-p_{(1)}^{E}-\cdots-p_{(n-1)}^{E}\right) .
\end{aligned}
$$

Let $\phi$ be the field in the momentum representation in the Lorentzian space-time. The relation between $\phi(p)$ and $\phi_{E}\left(p_{E}\right)$ can be found from the corresponding relations between the coordinate space fields $\widetilde{\phi}(x)$ and $\widetilde{\phi}_{E}\left(x_{E}\right)$,

$$
\widetilde{\phi}(x)=\widetilde{\phi}_{E}\left(x_{E}\right), \quad \phi(p)=\int d^{D} x e^{-i p \cdot x} \widetilde{\phi}(x), \quad \phi_{E}\left(p_{E}\right)=\int d^{D} x_{E} e^{-i p_{E} \cdot x_{E}} \widetilde{\phi}_{E}\left(x_{E}\right) .
$$

(3.4) and (3.8) now give,

$$
\phi(p)=-i \phi_{E}\left(p_{E}\right) .
$$

Using (3.9) and (3.4) we can express the right hand side of (3.7) as:

$$
\begin{gathered}
i^{n}(-i)^{n-1} \int d^{D} p_{(1)} \cdots d^{D} p_{(n-1)}\left\{\prod_{i=1}^{n-1} \phi\left(p_{(i)}\right)\right\} \phi\left(-p_{(1)}-\cdots-p_{(n-1)}\right) \\
=i \int d^{D} p_{(1)} \cdots d^{D} p_{(n)} \delta^{(D)}\left(p_{(1)}+\cdots+p_{(n)}\right) \prod_{i=1}^{n} \phi\left(p_{(i)}\right) .
\end{gathered}
$$

Identifying this as the contribution to $i S$, we see that the contribution to $S$ is given by:

$$
\int d^{D} p_{(1)} \cdots d^{D} p_{(n)} \delta^{(D)}\left(p_{(1)}+\cdots+p_{(n)}\right) \prod_{i=1}^{n} \phi\left(p_{(i)}\right) .
$$

As claimed, this is obtained from the Euclidean action by replacing the Euclidean momentum conserving delta function by the Lorentzian momentum conserving delta function, the euclidean momenta by Lorentzian momenta and the fields defined in the euclidean space by fields defined in the Lorentzian space. Generalization of these rules to tensor fields, and to cases where we have explicit momentum dependent functions multiplying the integrand, is straightforward.

With these rules, a real scalar function $f\left(p_{(i)}^{E}\right)$ of Euclidean momenta, multiplying the integrand in (3.7), will transform to a real scalar function of the Lorentzian momenta multiplying the integrand in (3.11), since the zeroth components of momenta occur in pairs in a scalar function. Note that in momentum space a real function means that the function is invariant under the simultaneous operation of complex conjugation and change of sign of all the momenta in its argument. On the other hand if the momenta are contracted to the index of a tensor field, then the extra $i$ from (3.1) will be compensated by the $i$ coming from the relation between the Lorentzian and the Euclidean version of the tensor field. Therefore these replacement rules do not generate any extra factor of $i$, and a real Euclidean action gets transformed to a real Lorentzian action. Conversely an imaginary term in the Euclidean action will generate an imaginary term in the Lorentzian action. 
The exception to this rule comes from terms involving the $\varepsilon$ tensor. We shall not discuss these terms in detail, but in physical examples such terms are accompanied by a factor of $i$ in the Euclidean action which goes away in the Lorentzian version.

Note that for this argument to work, it is essential that the function $f\left(p_{(i)}^{E}\right)$ is analytic in the complex $p_{(i)}^{E}$ plane. Otherwise one can find counterexamples. Let us for example suppose that the Euclidean theory has a term like $e^{-\left|p_{0}^{E}\right|}$ where $p^{E}$ denotes some combination of the momenta $p_{(i)}^{E}$. Since this remains invariant under simultaneous operation of complex conjugation and change of sign of the momenta, it is a real function. Now it follows from the discussion below (3.2) that positive $p_{0}^{E}$ is analytically continued to positive $p_{0}$ and negative $p_{0}^{E}$ is analytically continued to negative $p_{0}$. Therefore we see using (3.1) that $e^{-\left|p_{0}^{E}\right|}$ will analytically continue to the function $e^{i p_{0}}$ for positive $p_{0}$ and $e^{-i p_{0}}$ for negative $p_{0}$. This can be summarized by saying that the analytically continued function is $e^{-i\left|p_{0}\right|}$. This is not a real function, indicating that a real term in the Euclidean action leads to a complex term in the Lorentzian action. This can be traced to the non-analyticity of the function $f$ at $p_{0}^{E}=0$ that allows us to have terms of the form $e^{-\left|p_{0}^{E}\right|}$. In string field theory action such terms are absent since the possible non-analyticities of this kind can come from integration near the boundaries of the moduli space representing degenerate Riemann surfaces and these regions are excluded from the definition of the interaction terms of string field theory. Of course in the full amplitude we must also include contributions from the boundaries of the moduli space, and these contributions do lead to non-analyticity of the amplitude in the complex momentum plane. As a result, they produce imaginary parts of the amplitude for Lorentzian signature, consistent with cutting rules [6]. We shall illustrate this in the context of matrix model in section 7 . There, (7.7), representing a contribution to the two point closed string amplitude, is a non-analytic function of the external energy $\omega_{2}$ (after we strip off the momentum conserving delta function). In contrast, (7.16), representing a contribution to the action, is an analytic function of the energy.

In the presence of fermions, the relation between the reality properties of Euclidean and Lorentzian action is more complicated since the reality properties of the fermions themselves are different in the Euclidean and the Lorentzian theory. A given term in the Euclidean action involving fermions will be called real if after analytic continuation to Lorentzian signature (3.6), and imposing reality conditions on the fermions as given e.g. in [54], it gives a real term in the Lorentzian action.

Since the weight factor in the euclidean path integral measure is $e^{S_{E}}$, the contribution to an amputated $n$-point Green's function from a Feynman diagram with an $n$-point vertex and no internal propagator, as shown in figure 1, will be given directly by the integrand of the term in the action responsible for the interaction vertex, without any extra normalization factor (except for the symmetry factors $k$ ! for $k$ identical external particles). Similarly in the Lorentzian theory, where the weight factor is $e^{i S}$, the amputated $n$-point Green's function from a similar Feynman diagram will be given by $i$ times the integrand of the term in the action that produces the $n$-point vertex. This also gives the contribution to the S-matrix for on-shell external states. Therefore the contribution to the T-matrix from such a Feynman diagram will be given by the integrand of the term in the action without any extra normalization. Of course in all these cases there are also more compli- 


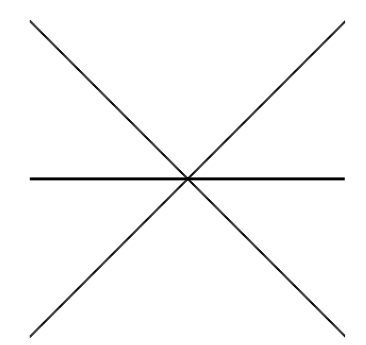

Figure 1. A Feynman diagram without any internal propagator, contributing to $n$-point amplitude.

cated Feynman diagrams with internal propagators that also contribute to the amplitudes. In the Euclidean theory we shall call the Green's functions as amplitudes whereas in the Lorentzian theory we shall call the T-matrix elements as amplitudes. Therefore for the contribution from diagrams without internal propagators, the amplitudes are related to the integrand of the terms in the action without extra normalization in both the Euclidean and the Lorentzian theories. From this it follows that the T-matrix elements in the Lorentzian theory are given by direct analytic continuation of the amplitudes in the Euclidean theory without any extra factor of $i$ and/or minus sign.

These observations will be useful while constructing the action of the closed string effective field theory from the amplitudes in the open closed string field theory.

\subsection{Reality properties of string field theory action}

In this section we shall discuss some results on the reality properties of string field theory actions.

We shall begin by reviewing the reality property of the ordinary interaction vertices of open closed string field theory, as defined in section 2. Since these interaction vertices are identical to the interaction vertices of the closed string field theory, they can be expressed as correlation functions of closed string vertex operators and other ghost insertions on Riemann surfaces without boundaries, integrated over a subspace of the moduli space of Riemann surfaces that avoids all degeneration points. Such integrals are manifestly finite and manifestly real with appropriate choice of reality condition on the string fields [5457]. Indeed a general feature of (super-)string perturbation theory is that all amplitudes are formally real, in the sense that they can be represented as integrals over the moduli space of Riemann surfaces with real integrands, and the imaginary parts, required for unitarity, come from having to regulate the divergences from the boundaries of the moduli spaces [14-22]. Since the region of integration describing the string field theory interaction vertices do not include the boundaries of the moduli spaces, they correspond to real terms in the action.

Some qualifications are necessary, however, on what one means by real integrand. As discussed before, in momentum space, reality of the integrand will mean that the integrand is invariant under simultaneous operation of complex conjugation and change of sign of all the external momenta. However, as discussed in [54, 56], even in the perturbative sector of closed string field theory, this does not hold - together with complex conjugation and 
change of sign of the external momenta, one also needs to include an involution operation on the moduli space of Riemann surfaces that changes the sign of the complex structure on the Riemann surface. After integration over part of the moduli space that is relevant for defining the interaction vertex, this gives us back the usual notion of reality of the interaction terms in the action.

The relevant string field theory in the presence of D-instantons is open closed string field theory which has both open and closed string fields. The interaction vertices of this theory are given by integrals of correlation functions of open and closed string vertex operators and world-sheet ghost fields on moduli spaces of Riemann surfaces with boundaries, and the integration runs over parts of the moduli space that do not include any degenerate Riemann surfaces. However, as alluded to at the end of section 2, and will be discussed in detail in section 4 and section 5 , we shall be working with the closed string effective action obtained by integrating out the open strings living on the D-instanton. The interaction vertices of this effective field theory will also be given by similar integrals, but now the external states will only have closed strings and the integration region will include degenerate Riemann surfaces associated with open string degeneration. Furthermore, the interaction vertices will carry overall multiplicative factors given by products of $\mathbf{N} e^{-C / g_{s}}$ factors described in section 2. This leads to the following possible sources of complex contribution to the closed string effective action:

1. The integrands that need to be integrated over the moduli spaces of Riemann surfaces may be complex.

2. $\mathbf{N}$ could be complex.

3. The divergences in the integrand from near the boundaries of the moduli spaces associated with open string degeneration may force us to define the integral via analytic continuation, and this may lead to imaginary terms in the effective action.

We can eliminate the first possibility by the following argument. The main ingredient in the proof of reality of the closed string field theory action is to establish a choice of basis states for the expansion of the off-shell closed string field in which all three point functions are real. Since the presence of boundaries on the Riemann surface can be represented by insertion of an off-shell closed string given by the boundary state associated with the D-brane, one would expect that as long as the boundary state is real in the same basis, the integrand that appears in the definition of the interaction vertex of closed string effective field theory is also real. ${ }^{5}$ Even if the boundary state is complex, as long as there is another D-instanton with complex conjugate boundary state, which has the same action and normalization constant $\mathbf{N}^{*}$, the net contribution to the closed string effective action will be real, since for every Riemann surface with boundaries ending on some combinations

\footnotetext{
${ }^{5}$ This argument shows the reality of the integrand in a region of the moduli space in which the effect of the boundaries can be regarded as disk one point functions connected to the rest of the diagram via closed string propagators. Since the integrand is non-singular in the interior of the moduli space, we would expect that this property can be made to hold in the entire moduli space by choosing the data on local coordinates at the punctures and the locations of the picture changing operators appropriately.
} 
of D-instantons, there is a another Riemann surface obtained by replacing each boundary state by its complex conjugate boundary state, and the sum of these two contributions to the integrand is still real. An example of this is provided by type IIB string theory, where D-instanton and anti-D-instanton produce complex conjugate contribution to the closed string effective action [8].

We shall formally summarize our results on reality, described above, as:

The reality postulate. The closed string effective action, obtained by integrating out all the open string modes on the D-instanton, can be formally expressed as the product of normalization constants $\mathbf{N} e^{-C / g_{s}}$ and integrals over the moduli spaces of Riemann surfaces with boundaries with real integrand. Therefore the imaginary contribution to the effective action comes either from the imaginary part of $\mathbf{N}$ or from boundaries of the moduli space associated with open strings degeneration, where the integrand may diverge, forcing us to define the integral in a different way.

As in the case of perturbative closed string field theory, some qualifications are necessary on what one means by real integrand. Since the world-sheet theory refers to some fixed locations of D-instantons in Euclidean space-time, the notion of reality, as stated, needs to be defined for fixed locations of the D-instantons, even though we'll eventually have to integrate over these locations. If we are working in Euclidean signature space-time, then the notion of reality is the same as that in the case of perturbative closed string field theory, i.e. the integrand is invariant under simultaneous operation of complex conjugation, change of sign of all the external momenta and an involution on the moduli space of Riemann surfaces that changes the sign of the complex structure on the Riemann surface. However, when external fermions are present, there is additional complication since the reality properties of fermions are different for Euclidean and Lorentzian signature. For this reason it is useful to define the notion of reality directly in the Lorentzian space by analytically continuing the integrand to Lorentzian signature, but still keeping the D-instanton positions at Euclidean time. This will ensure that eventually when we need to integrate over these locations, no extra factor of $i$ arises from having to rotate the integration contour of the D-instanton locations, and, more importantly, we do not need to worry about whether we might encounter poles while rotating the integration contour. Now, since from the Lorentzian perspective the D-instantons are located at imaginary time, the complex conjugation must also be accompanied by the change in sign of the instanton locations. Therefore after analytic continuation to Lorentzian signature, the reality of the integrand means that the integrand is invariant under the simultaneous operation of complex conjugation, reversal of sign of the external momenta, the involution on the moduli space involving change of sign of the complex structure on the Riemann surface and the reversal of sign of the D-instanton locations along the Euclidean time. ${ }^{6}$

\footnotetext{
${ }^{6}$ The coordinate describing the average time coordinate of all the D-instantons can be left out of this, since integration over this mode produces the overall Euclidean energy conserving delta function, which, following the algorithm described in section 3.1, gets mapped to Lorentzian energy conserving delta function.
} 
By working with the closed string effective action we avoid having to determine the reality condition on the open string fields on the D-instanton, since these fields are integrated out. The only exceptions are the zero modes which have to be 'integrated in' for dealing with the divergences coming from the boundaries of the moduli spaces, and then integrated separately at the end. The effect of integration over these modes will be discussed in subsequent sections.

\section{One instanton effective action and its reality properties}

In this section we shall discuss the construction of the one instanton effective action of closed strings and analyze its reality properties and the cutting rules.

\subsection{Effective action}

In an ordinary quantum field theory, if we want to integrate out a set of fields $U^{c}$ and construct the effective action of the complementary set of fields $U$, we define the $n$-point interaction vertex of the effective field theory to be the sum of all connected diagrams for $n$-point amputated Green's function that do not have any propagator of a field in the set $U$. In the current problem $U$ stands for the set of closed string fields and $U^{c}$ stands for the set of open string fields on the D-instanton. Formally, the procedure for constructing the interaction vertex of the closed string effective field theory is similar, except that we also sum over disconnected contribution to the Green's function as long as each connected component is made of D-instanton type interaction vertices of the original open closed string field theory. The inclusion of disconnected diagrams in the definition of the interaction vertex of the effective field theory may be unfamiliar, but is necessary due to the fact that (1) the individual connected components do not satisfy momentum conservation and (2) irrespective of how many connected components a diagram has, we have a single factor of $\mathbf{N} e^{-C / g_{s}}$ multiplying the amplitude. This is also reflected in the fact that the amplitude is to be evaluated in the open string zero mode background which needs to be integrated at the end after summing over disconnected diagrams. In fact we shall also include 0-point D-instanton type vertices with no external open and closed strings but just Riemann surfaces with boundaries. As usual, these zero point interaction vertices will involve integration over subspaces of the moduli spaces of the corresponding Riemann surfaces that are not covered by Feynman diagrams with internal open and / or closed string propagators. The only exception will be the annulus diagram that is included in the overall normalization constant $\mathbf{N}$. Figure 2 shows examples of some closed string four point amplitudes in the original open closed string field theory that become part of the four point interaction vertex in the closed string effective field theory. We shall call the ordinary $n$ point interaction vertex of the original open closed string field theory the ordinary $n$-point interaction vertex of the effective field theory, and the sum of all other contributions to the $n$-point interaction vertex of the effective field theory the $\mathrm{D}$-instanton type interaction vertex. It then follows from the Feynman rules of open closed string field theory that the

However the differences between the Euclidean time coordinates of different D-instantons will have to change sign. 


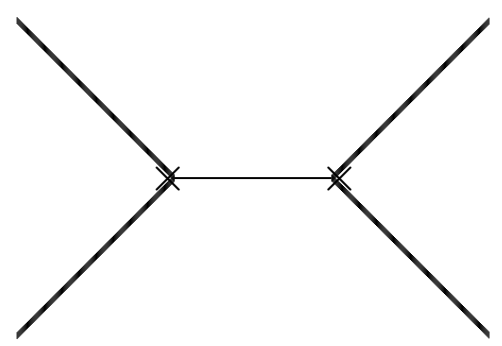

(a)

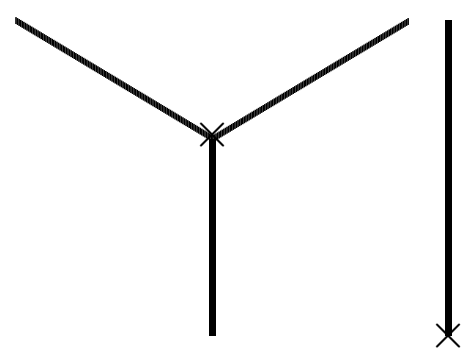

(b)

Figure 2. Examples of amplitudes in the original open closed string field theory on a single Dinstanton that become part of the interaction vertex of the closed string effective field theory. The thick lines represent closed string propagators, the thin lines represent open string propagators and the $\times$ 's denote D-instanton type interaction vertex.

D-instanton type interaction vertex of the effective field theory will be accompanied by a factor of $\mathbf{N} e^{-C / g_{s}}$ irrespective of how many D-instanton type interaction vertices of the original theory it has. We shall show in section 4.3 that the action of the closed string effective field theory constructed this way satisfies the quantum BV master equation [25] and therefore has the gauge invariance required for the consistency of the theory.

We can also give the relation between the Feynman diagrams built from this effective field theory and those built from the original open closed string field theory. Given any Feynman diagram of the original open closed string field theory, we draw an auxiliary Feynman diagram in which we fuse all the D-instanton type interaction vertices into a single composite vertex, and also collapse all the open string propagators (which must begin and end at the D-instanton type vertices) into this composite vertex. We keep all the closed string propagators displayed as usual. The composite vertex constructed this way is part of the D-instanton type interaction vertex of the closed string effective field theory. This has been illustrated in figure 3. Note that there is only one composite vertex, i.e. only one D-instanton type vertex of the closed string effective field theory in the entire diagram. This reflects the fact that since we are considering single D-instanton amplitude, we allow only one power of $e^{-C / g_{s}}$. Therefore the D-instanton type interaction vertex of the closed string effective field theory, that we get by integrating out the open string fields, is used only once. As will be discussed in section 5, diagrams where the vertex is used multiple times are part of the multi-instanton contribution to the amplitude. It is also useful to note that if in the original Feynman diagram of the open closed string field theory none of the D-instanton type vertices were connected to an external closed string, either directly or via internal closed string propagators, then after collapsing them into the composite vertex, we would get a disconnected bubble diagram of the closed string effective field theory. However, such diagrams are excluded from the beginning in the open closed string field theory Feynman rules due to cancellation between the numerator and the denominator factors in the path integral [53]. 

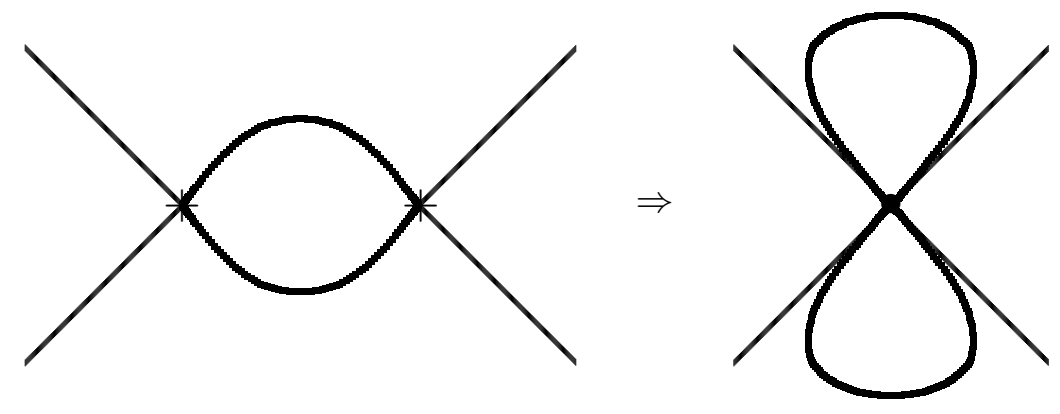

Figure 3. Translating a Feynman diagram of the original open closed string field theory (left) into a Feynman diagram of the closed string effective field theory (right). The interaction vertices in the left diagram are D-instanton type interaction vertices of the open closed string field theory and the vertex labelled by $\bullet$ on the right diagram is a D-instanton type interaction vertex of the closed string effective field theory. The propagators displayed in this diagram are closed string propagators. If the original diagram had any open string propagator, it would have been fused into the interaction vertex of the effective field theory.

\subsection{Divergences}

This construction of the closed string effective field theory described above suffers from various divergences that need to be treated carefully [53]. The ordinary interaction vertices of the effective field theory, being identical to the ordinary interaction vertices of the original open closed string field theory, have the interpretation of correlation functions of closed string vertex operators and other ghost insertions on Riemann surfaces with boundaries, integrated over a subspace of the moduli space of Riemann surfaces that avoids all degeneration points. Such integrals are manifestly finite. Let us now consider the Dinstanton type interaction vertex in the closed string effective field theory. This is also given by integrals of correlation functions of closed string vertex operators and world-sheet ghost fields on moduli spaces of Riemann surfaces, but the Riemann surfaces have one or more boundaries with D-instanton boundary condition. The part of the moduli space of these Riemann surfaces over which we need to integrate for computing the effective action now includes one or more boundaries associated with open string degeneration. Therefore the integrals could diverge from these regions, requiring special treatment. This has been discussed in detail in [53], but we shall summarize the main points below.

1. We may get divergences of the form $\int_{0}^{1} d q q^{-\alpha-1}$ where $q$ is related to the Schwinger parametrization of the open string propagators, and is usually referred to as the sewing parameter due to its role in the world-sheet description of the amplitude. The divergences arising from the terms with $\alpha>0$ can be traced to the propagation of open string tachyons. Open closed string field theory instructs us to use the replacement rule,

$$
\int_{0}^{1} d q q^{-\alpha-1} \rightarrow-\alpha^{-1}
$$

for dealing with these divergences. 
2. We may also get logarithmic divergences of the form $\int_{0}^{1} d q q^{-1}$. These can be traced to the result of integration over the open string zero modes. The prescription described in [53] was to remove these contributions and then integrate over the zero modes separately. The procedure for removing these contributions may be formally stated as:

$$
\int_{0}^{1} d q q^{-1} \rightarrow 0
$$

Since we leave the zero modes unintegrated, we need to treat them as external states. Following this algorithm we arrive at the Wilsonian effective action, expressed as function of closed string fields and open string zero mode fields.

We shall now list the zero modes over which we have to carry out the integrals, and the strategy we follow to compute these integrals:

1. There are open string zero modes, which, after field redefinition, can be related to the collective coordinates of the D-instanton describing the location of the instanton in Euclidean space-time. The effect of integration over the collective coordinates will be described shortly. The field redefinition that relates the open string modes to the collective coordinates gives rise to Jacobian in the integration measure, which can be regarded as additional multiplicative contribution to the closed string effective action. This could include, in particular, the closed string moduli fields including the dilaton, leading to a moduli dependent multiplicative factor in the effective action [36].

2. There is an open string zero mode from the ghost sector associated with the rigid $U(1)$ gauge transformation on the D-instanton. After an appropriate field redefinition, the path integral over this zero mode may be regarded as division by the volume of the $\mathrm{U}(1)$ gauge group. Since this is a finite constant that can be absorbed into the definition of the normalization constant $\mathbf{N}$, we shall drop this integral from our analysis. However the Jacobian that arises from the required change of variable gives rise to additional multiplicative contribution to the closed string effective action.

3. Finally, due to the breakdown of the Siegel gauge, there is an extra auxiliary open string field from the ghost sector and we have to explicitly evaluate its contribution to the Feynman diagrams. This gives extra terms that are finite and computable.

4. The integration over the collective coordinates is done at the end, and produces the momentum conserving delta function that involves total momentum of the external closed strings in all the D-instanton type vertices. Therefore this produces the momentum conserving delta function at the D-instanton type vertex of the closed string effective field theory. The original integration is done in Euclidean space, producing the delta function with argument given by total Euclidean momentum. According to (3.7), (3.11) this translates to a momentum conserving delta function in the Lorentzian space.

5. In superstring theories there will also be fermionic collective coordinates that need to be integrated over. This means that when we construct the D-instanton type 
interaction vertex of the effective field theory, we need to sum over Feynman diagrams of the original open closed string field theory that have not only external closed string states, but also certain number of open string states representing the zero mode fields. There can be additional contribution to the effective action from the Jacobian associated with the change of variables from the open string fields to the fermionic collective coordinates.

From the analysis above, we see that the D-instanton type interaction vertex of the closed string effective field theory has the usual momentum conserving delta function. Furthermore a single vertex of this type is accompanied by a single factor of $\mathbf{N} e^{-C / g_{s}}$. This removes the first hurdle in the proof of cutting rules in this effective field theory. We shall analyze the reality of the effective action in section 4.4 .

\subsection{Gauge invariance}

In string field theory, the kinetic term in the action is given by the matrix element of the world-sheet BRST operator between a pair of string fields. If $P$ is any projection operator that commutes with the BRST operator, then there is a way to integrate out all $P$ non-invariant fields and write down an effective action for the $P$ invariant fields, both in the classical theory $[23,24,26]$ and in the quantum theory [25]. The resulting string field theory preserves all the algebraic structures of the original theory and satisfies the quantum Batalin-Vilkovisky (BV) master equation. Since the BRST operator in the combined Hilbert space of open and closed strings is block diagonal, we can use this procedure to integrate out all the open string modes on the D-instanton and write down an effective action for closed string fields.

There are however a few differences between the process of integrating out a subset of fields described in [25] and the one described here, due to the inclusion of disconnected diagrams, and also due to the presence of the normalization factor $\mathbf{N} e^{-C / g_{s}}$. To understand this difference, let us denote by $S_{0}$ the action of purely closed string field theory, and by $S_{1}$ the additional term in the action that we get by integrating out the open string modes in the sense of [25]. $S_{1}$ will be given by appropriate correlation functions of closed string vertex operators on the connected world-sheet with boundaries, integrated over appropriate regions of the moduli spaces of Riemann surfaces that include open string degeneration but no closed string degeneration. We shall proceed by ignoring possible divergences in $S_{1}$ from the boundaries associated with open string degeneration, but will comment on this at the end of this subsection. Then the effective action that would appear in the analysis of [25] would be $S_{0}+S_{1}$, and it would satisfy the BV master equation $[58,59]$

$$
\frac{1}{2}\left\{S_{0}+S_{1}, S_{0}+S_{1}\right\}+\Delta\left(S_{0}+S_{1}\right)=0
$$

where $\{A, B\}$ denotes the anti-bracket of $A$ and $B$ and $\Delta$ is the BV Laplacian:

$$
\{A, B\}=\frac{\partial_{r} A}{\partial \psi^{s}} \frac{\partial_{l} B}{\partial \psi_{s}^{*}}-\frac{\partial_{r} A}{\partial \psi_{s}^{*}} \frac{\partial_{l} B}{\partial \psi^{s}}, \quad \Delta A=\frac{\partial_{r}}{\partial \psi^{s}} \frac{\partial_{l}}{\partial \psi_{s}^{*}} A .
$$


Here $\psi^{s}$ are the field variables, $\psi_{s}^{*}$ are the conjugate anti-fields carrying opposite grassmann parity, and $\partial_{l}$ and $\partial_{r}$ denote left and right derivatives respectively. Since $S_{0}$ itself satisfies the BV master equation, we actually have two equations:

$$
\frac{1}{2}\left\{S_{0}, S_{0}\right\}+\Delta S_{0}=0,
$$

and

$$
\left\{S_{0}, S_{1}\right\}+\frac{1}{2}\left\{S_{1}, S_{1}\right\}+\Delta S_{1}=0 .
$$

We have used the fact that $\{A, B\}$ is invariant under the exchange of $A$ and $B$ if both $A$ and $B$ are grassmann even.

In contrast, the effective action that we have here includes contribution from disconnected world-sheets, as long as every disconnected component has a boundary ending on the D-instanton. Furthermore, all diagrams with boundaries will be multiplied by a factor of

$$
K \equiv \mathbf{N} e^{-C / g_{s}} .
$$

Since the sum of the disconnected diagrams may be represented as $e^{S_{1}}-1$, the total effective action may be written as

$$
S_{\text {total }}=S_{0}+K\left(e^{S_{1}}-1\right)+\mathcal{O}\left(K^{2}\right)
$$

where the $\mathcal{O}\left(K^{2}\right)$ terms denote multi-instanton effective action. We now need to check if $S_{\text {total }}$ satisfies the BV master equation. For this we compute:

$$
\frac{1}{2}\left\{S_{\text {total }}, S_{\text {total }}\right\}=\frac{1}{2}\left\{S_{0}, S_{0}\right\}+K\left\{S_{0}, S_{1}\right\} e^{S_{1}}+\mathcal{O}\left(K^{2}\right) .
$$

We also have

$$
\Delta S_{\text {total }}=\Delta S_{0}+K \Delta e^{S_{1}}=\Delta S_{0}+K\left(\frac{1}{2}\left\{S_{1}, S_{1}\right\}+\Delta S_{1}\right) e^{S_{1}},
$$

where we have used the standard identity involving $\Delta$ and the anti-brackets that follows from (4.4). Adding (4.9) and (4.10) and using (4.5), (4.6), we get,

$$
\frac{1}{2}\left\{S_{\text {total }}, S_{\text {total }}\right\}+\Delta S_{\text {total }}=\mathcal{O}\left(K^{2}\right) \text {. }
$$

As will be demonstrated in section 5.4, the order $K^{2}$ terms will cancel against the contribution from the 2-instanton effective action. This shows that the one instanton effective action satisfies the desired master equation.

Note that in the above analysis we have ignored the issues of divergences and have pretended that the effective action can be computed as if we can represent its interaction terms as appropriate integrals over the moduli spaces of Riemann surfaces with no modification. Therefore the proof of gauge invariance is formal. However, since the treatment of divergences merely reorganizes the way we carry out the integration over the string fields, and does not add or subtract any ad hoc gauge non-invariant terms, we do not expect this procedure to cause breakdown of gauge invariance. 


\subsection{Reality of the effective action}

We shall now turn to the study of reality properties of the effective action. The situation here is different from the case of effective field theory obtained by integrating out heavy physical fields, where we do expect the effective action to acquire imaginary part above a certain momentum threshold, describing the possibility of production of heavy states and violation of unitarity in the light sector alone. Here the degrees of freedom that have been integrated out are open strings that are not allowed asymptotic states, and we need the reality condition to hold exactly for the proof of unitarity.

We shall begin with the ordinary interaction vertices of the theory. Since these interaction vertices are identical to the ordinary interaction vertices of the closed string field theory, they generate real terms in the closed string effective action [54, 56, 57].

The D-instanton type interaction vertices in the open closed string field theory are given by integrals of correlation functions of open and closed string vertex operators and world-sheet ghost fields over the moduli spaces of Riemann surfaces with boundaries, and the integration runs over parts of the moduli space that do not include any degenerate Riemann surfaces. Formally, after integrating out the open string modes, the interaction vertices of closed string effective field theory will also be given by similar integrals, but now the vertex operators will only be of closed strings and the integration region will include degenerate Riemann surfaces associated with open string degeneration. According to the reality postulate of section 3.2 whose validity we shall assume, the integrand itself will be real after factoring out the overall normalization constant $\mathbf{N}$, and the possible imaginary contributions can only arise from having to carefully treat the divergences that arise from the boundaries of the moduli spaces associated with open string degeneration. This, and the normalization constant $\mathbf{N}$, will be the only source of violation of reality of the Lorentzian effective action for closed strings, obtained by integrating out the open strings.

Let us therefore consider the effect of divergences from the boundaries of the moduli spaces. (4.1) gives the procedure for dealing with power law divergences associated with tachyonic internal states. We see that this replaces a formally real but divergent integral by a real number. Therefore this replacement does not affect the reality property of the amplitude given as integrals over the moduli spaces of Riemann surfaces. On the other hand, the first step for dealing with logarithmically divergent integrals is to use the replacement rule (4.2) that replaces a formally real but divergent integral by 0 . This also does not affect the reality property of the amplitude. The second step is to actually carry out the integration over the zero modes following the procedure described in section 4.1. We shall now examine if these could lead to violation of the reality condition.

First let us consider the terms in the effective action that arise from the two Jacobians associated with field redefinition of the collective mode and the ghost zero mode, and the contribution from the auxiliary ghost field propagators. It can be shown that these computations can all be reduced to the computation of certain amplitudes in the original open closed string field theory, and are free from all divergences [60]. Therefore by the general result that string theory amplitudes are real unless they are divergent, these contributions do not affect the reality property of the interaction vertices. The only exception is the 
possibility of an overall complex constant factor - since we have not determined the reality condition on the open string fields, we can redefine them by multiplicative complex constants leading to a multiplicative constant in the Jacobians. This will only affect the overall normalization constant $\mathbf{N}$, which in any case cannot be computed with the present technology and will have to be determined by other considerations.

We now turn to the effect of the final integration over the collective coordinates. As already discussed, this produces the momentum conserving delta function involving the total euclidean momentum carried by all the external states of the interaction vertex of the effective field theory. According to (3.7), (3.11) this translates to a momentum conserving delta function in the Lorentzian space. Therefore this integral also does not affect the reality property of the interaction vertex.

In superstring theories there will also be fermionic collective coordinates that need to be integrated over. This means that in the amplitude needed to construct the interaction vertex, we need to insert the vertex operators of open string fermion zero modes besides the usual closed string vertex operators. Furthermore, since open strings live in the Euclidean theory, this computation needs to be performed in the Euclidean theory. This could be potentially problematic since the reality property of spinors in the Euclidean and the Lorentzian signature spaces are different. We can avoid this problem as follows. If there are $2 N$ real fermion zero modes, we can combine them into $N$ complex conjugate pairs. Now the result of insertion of a given complex conjugate pair of zero modes in a given Feynman diagram can be regarded as part of the coefficient of the $q^{-1}$ term in an auxiliary Feynman diagram in which we replace the insertion of the complex conjugate pair of zero modes by an open string propagator with sewing parameter $q$. This exercise can be repeated for each complex conjugate pair of zero modes leading to $N$ open string propagators with sewing parameters $q_{1}, \cdots, q_{N}$. At the end we have an amplitude with only external closed strings, and the amplitude with open string zero modes is part of the coefficient of the $\prod_{i=1}^{N} q_{i}^{-1}$ term in the amplitude. Since the integrand of the amplitude with external closed strings is real by our reality postulate, the coefficient of $\prod_{i=1}^{N} q_{i}^{-1}$ in the integrand is also real, establishing the reality of the amplitude with $2 N$ open string zero mode field insertions. This argument breaks down if the D-instanton has complex boundary state, but in that case the contribution from the D-instanton with complex conjugate boundary state will provide the complex conjugate contribution, restoring the reality of the effective action.

Of course, due to the various issues involving differences in the reality properties of fermions in the Euclidean and Lorentzian spaces, there may be extra factors of $i$ coming from integration measure over the fermionic zero modes. However such factors are common to all amplitudes and may be absorbed into the overall normalization constant $\mathbf{N}$.

Therefore we conclude that the action of the closed string effective field theory, obtained after integrating out the open string fields on the D-instanton, is real provided the normalization constant $\mathbf{N}$ is real. Since at present we do not have a systematic procedure for determining $\mathbf{N}$ from first principles, we have to rely on indirect means for determining $\mathbf{N}$ and checking its reality. These methods include comparison with matrix model results for two dimensional string theory and use of S-duality for type IIB string theory. In the former case $\mathbf{N}$ will turn out to be imaginary whereas for type IIB superstring $\mathbf{N}$ for 
D-instanton and anti-D-instanton can be shown to be complex conjugates of each other, leading to real effective action. This is evident from the reality of the instanton induced term in the effective action needed for consistency with S-duality [8]. The limited data available to us, as well as the fact that $\mathbf{N}$ is formally given by the one loop determinant of open string modes on the D-instanton, suggests that the violation of the reality of $\mathbf{N}$ is correlated with the presence of the tachyonic mode on the D-instanton.

\subsection{Cutting rules and unitarity}

Since for real $\mathbf{N}$ the interaction vertices of the closed string effective field theory satisfy the usual momentum conservation and reality conditions, we can apply the results of [6] to conclude that the anti-hermitian part of any amplitude is given by the sum over all the cut diagrams of the Feynman diagrams of the closed string effective field theory. We can now translate the cutting rules of this effective field theory into the cutting rules of the original open closed string field theory as follows. In the effective field theory with the usual cutting rules, the cut does not pass through any vertex. Therefore the single D-instanton type vertex present in the entire Feynman diagram must lie on one side of the cut. When we interpret this in terms of the Feynman diagrams in the original open closed string field theory, this implies that all the D-instanton type vertices must lie on one side of the cut. Furthermore, since all internal open string propagators must have both ends attached to a D-instanton type interaction vertex, no open string propagator is cut. As discussed in section 2, this is exactly the kind of cutting rules we need for the proof of unitarity of D-instanton induced amplitudes.

The cutting rules are one of the ingredients in the proof of unitarity. In a gauge theory like string field theory, we also need to show that in the cut propagator the contribution from the unphysical states cancel so that the sum over cut diagrams can be interpreted as the right hand side of (2.1) with the sum over $n$ running over physical states only. For closed string field theory this was proved in [52] using the BV formalism. Since the closed string effective field theory also satisfies the BV master equation, the same proof will hold for this theory as well.

\section{Multi-instanton contribution to the effective action and its reality properties}

We shall now analyze the $k$ D-instanton contribution to the closed string effective action and study its reality properties. We shall first consider the case of $k$ different D-instantons, carrying normalization factors $\mathbf{N}_{1} e^{-C_{1} / g_{s}}, \cdots, \mathbf{N}_{k} e^{-C_{k} / g_{s}}$, and then discuss some additional subtleties that may arise for identical D-instantons.

\subsection{Construction of the effective action ignoring the zero modes}

First we shall ignore the effect of the zero modes and divergences from the boundaries of the moduli spaces of Riemann surfaces and study how the Feynman diagrams of open-closed string field theory can be organized into Feynman diagrams of closed string effective field 
theory. This will help us read out the interaction vertices of the closed string effective field theory. Let us first consider the case $k=2$.

1. In this case the Riemann surfaces corresponding to the amplitudes have two types of D-instanton boundary condition, corresponding to the first or the second D-instanton. In the open closed string field theory there are now three different types of D-instanton interaction vertex, type 1 , type 2 and type 12, reflecting the fact that associated Riemann surfaces have boundaries of type 1, type 2 and types 1 and 2 . As in section 4 , we must also include in this discussion the zero point vertices, associated with Riemann surfaces with boundaries but no punctures, and disconnected diagrams.

2. Since for two instanton amplitudes the underlying Riemann surfaces must have at least one boundary of each type, we can classify the Feynman diagrams in the open closed string field theory into two possible types - (a) those carrying interaction vertices of type 1 and type 2, but not of type 12 and (b) those carrying at least one interaction vertex of type 12 and possibly other interaction vertices of any type.

3. Let us now compare this with the diagrams generated by the closed string effective field theory with two insertions of single D-instanton type vertex constructed in section 4 , one of type 1 and the other of type 2 . It is straightforward to see that when we reinterpret this as Feynman diagrams in the original open closed string field theory, then these generate all the diagrams of type (a) but none of the diagrams of type (b). Therefore we must interpret the Feynman diagrams of type (b) as being generated by a new interaction vertex of closed string effective field theory, obtained by fusing all the D-instanton type interaction vertex and the open string propagators into a single composite vertex.

4. Put another way, the new $n$-point interaction vertex of the closed string effective field theory will contain all $n$-point connected and disconnected amplitudes of the original open closed string field theory that use at least one 12 type interaction vertex, and arbitrary number of possibly other D-instanton type vertices and internal open string propagators, but no internal closed string propagator or ordinary interaction vertex.

The annulus diagrams require special care. These diagrams do not carry any factor of $g_{s}$ and therefore must be resummed as an exponential multiplying the integrand. There are different types of annulus diagrams depending on the boundary conditions at the two boundaries. The diagrams where both boundaries carry the same boundary condition can be exponentiated and their contribution can be absorbed into a factor of $\mathbf{N}_{1} \mathbf{N}_{2}$ in the overall normalization. The annulus diagrams with the two boundaries having two different boundary conditions must be regarded as a contribution to the 12 type zero point function, and therefore Feynman diagrams containing one or more factors of such annuli and possibly other D-instanton type vertices and open string propagators, but no internal closed string propagator or ordinary interaction vertex, must be included in the contribution to the two 


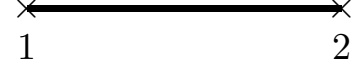

Figure 4. A closed string exchange diagram that gives part of the annulus diagram with different boundary conditions on the two boundaries.

instanton interaction vertex in the effective field theory. There are however some subtleties that we discuss below:

1. In open closed string field theory, part of this annulus diagram comes from a Feynman diagram where a pair of closed string one point functions on the disk, with 1 and 2 type boundary conditions, are connected by a closed string propagator. This has been shown in figure 4 . So only part of the annulus contribution, that is not included in figure 4 , should be counted as a contribution to the 12 type zero point interaction vertex in open closed string field theory, and this is the part that needs to be included in the construction of the 2-instanton interaction vertex of closed string effective field theory following the procedure described above. If we regard the annulus as a cylinder and denote by $2 t$ the ratio of the circumference to the height of the cylinder, then the Feynman diagram of figure 4 covers the integration range $0 \leq t \leq a$ where $a$ is some real number. The precise value of $a$ depends on the choice of the off-shell closed string one point interaction vertex. Therefore the part of the annulus contribution that should be included in the definition of the two instanton vertex of the closed string effective field theory is given by:

$$
A_{12}=\int_{a}^{\infty} d t t^{-1} Z_{12}(t)
$$

where $Z_{12}(t)$ is the partition function of the 12 open strings:

$$
Z_{12}(t)=\operatorname{Tr}_{12} e^{-2 \pi t L_{0}} .
$$

Usually we would have a factor of $1 / 2$ in (5.1), but we have included in (5.1) also the contribution from 21 open strings, assuming that $Z_{21}=Z_{12}$.

2. Now consider a general Feynman diagram of the open closed string field theory with external closed strings but no internal closed string or ordinary interaction vertices and no factor of the annulus diagram. If the diagram under consideration has components with 12 type interaction vertex, then it is part of the contribution to the 2 -instanton interaction vertex of the closed string effective field theory, and we must multiply this by $e^{A_{12}}$ to take into account the effect of all possible insertions of annulus diagrams with two boundaries lying on two different instantons. On the other hand if the original diagram does not have any 12 type interaction vertex, then it is not a part of the 2-instanton interaction vertex of the closed string effective field theory. Now we should multiply this by $e^{A_{12}}-1$ to get a contribution to the interaction vertex of the closed string effective field theory. Diagrammatically this may be interpreted by saying that a Feynman diagram in the open closed string field theory containing 
only external closed strings and no internal closed strings or ordinary interaction vertices, can be regarded as a contribution to the 2-instanton interaction vertex of the closed string effective field theory if after fusing the interaction vertices and / or annuli sharing common boundary type, we are left with a single composite vertex.

With this prescription the two instanton interaction vertex in the closed string effective field theory will have an overall multiplicative factor of $\mathbf{N}_{1} \mathbf{N}_{2} e^{-\left(C_{1}+C_{2}\right) / g_{s}}$, and the rest of the contribution to the interaction vertex will have to be computed explicitly following the algorithm described above. We shall call this the 12 type interaction vertex of the closed string effective field theory.

Next consider the case $k=3$. In this case the Riemann surfaces associated with the amplitudes have three different types of boundaries, and the corresponding open closed string field theory has many different types of D-instanton type vertices - types 1, 2, 3, $12,23,13$ and 123. Now given a general Feynman diagram in this theory, the question that we need to ask is if this diagram can be associated with a Feynman diagram of the closed string effective field theory using the interaction vertices that have already appeared in the analysis of one and two instanton amplitudes. If the answer is in the negative then we need a new interaction vertex of the closed string effective field theory to generate this diagram in the open closed string field theory. We shall first illustrate this with some examples and then give the general result.

Let us suppose that the Feynman diagram of the open closed string field theory has D-instanton type interaction vertices of type 1,2, 12 and 3. Then we can get this from a Feynman diagram of the closed string effective field theory with one 2-instanton interaction vertex and one 1-instanton interaction vertex, obtained as follows. We fuse all the 1, 2 and 12 type interaction vertices, as well as any open string propagator connected to these vertices, into a single composite vertex and interpret this as part of a 2-instanton interaction vertex of the closed string effective field theory. We also fuse all the 3 type interaction vertices, as well as any open string propagator connected to these vertices, into a single composite vertex and interpret this as part of a 1-instanton vertex of the closed string effective field theory. Therefore for this class of diagrams we do not need to introduce a new interaction vertex in the closed string effective field theory. On the other hand if the Feynman diagram of the original open closed string field theory contained 12 and 23 type vertices, or 123 type vertex, then there will be no way to get this from the 1 and 2-instanton interaction vertices of closed string effective field theory. We need to fuse all the interaction vertices of the Feynman diagram, as well as any open string propagator connected to these vertices, into a single composite vertex and interpret this as part of a 3 -instanton interaction vertex of the closed string effective field theory.

Based on these examples, we see that a 3 -instanton interaction vertex of the closed string effective field theory with $n$ external closed strings is obtained by summing over all Feynman diagrams of open closed string field theory with $n$ external closed strings, satisfying the following properties:

1. The diagram can have internal open string propagators and D-instanton type vertices of the open closed string field theory, but cannot have any ordinary interaction vertex or internal closed string propagator. 
2. If we fuse each pair of interaction vertices sharing a common boundary type, and repeat this for all pairs, then at the end we should be left with a single composite vertex.

We now turn to the discussion of annulus diagrams which we have ignored so far. Annuli with the same boundary condition on the two boundaries are included in the overall normalization factor $\mathbf{N}_{1} \mathbf{N}_{2} \mathbf{N}_{3} e^{-\left(C_{1}+C_{2}+C_{3}\right) / g_{s}}$. Now consider a diagram in the open closed string field theory with no ordinary interaction vertex and no internal closed string propagator. Its contribution to the 3 -instanton vertex of closed string effective field theory is determined as follows.

1. If after following the rules described above for fusing the interaction vertices we are left with a single composite vertex, then the contribution from the diagram is multiplied by a factor of $\exp \left[A_{12}+A_{23}+A_{13}\right]$, with $A_{i j}$ defined as in (5.1), to determine its contribution to the 3 -instanton vertex.

2. If after following the rules for fusion the diagram has two composite vertices, one containing all world-sheet boundaries of type $i$ and $j$ and the other containing all world-sheet boundaries of type $k$, then it should be multiplied by $e^{A_{i j}}\left(e^{A_{i k}+A_{j k}}-1\right)$, so that we are forced to include at least one factor of $A_{i k}$ or $A_{j k}$ from the annulus diagram. Diagrams with only $A_{i j}$ insertions are already present in the Feynman diagram of the closed string effective field theory with one insertion of two instanton vertex of type $i j$ and one insertion of two instanton vertex of type $k$.

3. Finally if a diagram in open closed string field theory has only interaction vertices of type 1, 2 and 3 so that it has three composite vertices after we use the rules for fusion, then it needs to be multiplied by

$$
\left(e^{A_{12}}-1\right)\left(e^{A_{23}}-1\right)\left(e^{A_{13}}-1\right)+\left(e^{A_{12}}-1\right)\left(e^{A_{23}}-1\right)+\left(e^{A_{23}}-1\right)\left(e^{A_{13}}-1\right)+\left(e^{A_{12}}-1\right)\left(e^{A_{13}}-1\right) .
$$

The first term represents the contribution where there is at least one annulus of each type, 12, 23 and 13 . The second term represents the contribution where there is at least one annulus of type 12 and one annulus of type 23, but no annulus of type 13 . The other two terms are obtained as permutations of the second term. This includes all diagrams containing one of the following configurations: 1) at least one 12 and one 23 type annulus, 2) at least one 13 and one 23 type annulus and 3) at least one 12 and one 13 type annulus.

We are now ready to generalize this to $k$-instanton amplitudes. In section 2 we have already introduced the notion of a label $\left(i_{1}, \cdots, i_{\ell}\right)$ for each interaction vertex of open closed string field theory specifying the type of boundary segments that the Riemann surface associated with the vertex has. For a given Feynman diagram in this open-closed string field theory, we now introduce an auxiliary diagram, in which we fuse each pair of interaction vertices of the original diagram, that share a label, into a composite vertex, and also fuse all the open string propagators connected to the original vertices into the 
composite vertex. For example, if the original Feynman diagram had interaction vertices of types 12, 23, 36 and 45, then in the auxiliary diagram the vertices of type 12, 23 and 36 will be fused into a single composite vertex, and the vertices of type 45 will be fused together (if there are more than one such vertices) to form a second composite vertex. If the auxiliary Feynman diagram, constructed this way, has more than one composite vertex, then it can be regarded as coming from the Feynman diagram of the closed string effective field theory based of $\ell$ instanton vertices with $\ell<k$. If on the other hand the auxiliary diagram has only one composite vertex, i.e. if all the D-instanton type vertices of the original Feynman diagram are fused into a single composite vertex, then it needs to be interpreted as coming from a Feynman diagram of closed string effective field theory that uses a new vertex - the $k$-instanton interaction vertex. Conversely, a $k$-instanton interaction vertex of the closed string effective field theory, with $n$ external closed strings, is obtained by summing over all Feynman diagrams of open closed string field theory with $n$ external closed strings, satisfying the following properties:

1. The diagram can have internal open string propagators and D-instanton type vertices of the open closed string field theory, but cannot have any ordinary interaction vertex or internal closed string propagator.

2. If we fuse all pairs of interaction vertices sharing a common boundary type, then at the end we should be left with a single composite vertex.

This is exactly analogous to the situation for $k=2$ and $k=3$.

We need to augment this rule by giving the algorithm for including annulus diagrams with different boundary conditions. Let us call an annulus with $i$ type boundary condition on one boundary and $j$-type boundary condition on the other boundary an $i j$ type annulus. Given a Feynman diagram in the open closed string field theory with only external closed strings and no internal closed strings and ordinary interaction vertices, we must sum over those annulus insertions for which, after fusing all the interaction vertices and annuli sharing a common label, we are left with a single composite vertex. The required insertions may be expressed as combinations of $e^{A_{i j}}$ with $1 \leq i<j \leq k$. The overall multiplicative factor for this vertex will be given by $\prod_{i=1}^{k}\left\{\mathbf{N}_{i} e^{-C_{i} / g_{s}}\right\}$, with the $\mathbf{N}_{i}$ 's including contributions from the annuli whose both boundaries have the same boundary condition.

\subsection{Effect of divergences and open string zero modes}

Let us now turn to the analysis of possible divergences. We begin with the configuration where the D-instantons are widely separated so that the open strings connecting a pair of D-instantons have positive mass ${ }^{2}$ and do not cause any divergences. In this case the divergences will arise from tachyonic modes and zero modes living on individual D-instantons. Tachyons can be handled using the replacement rule (4.1) and will not require any further discussion. However the zero modes require special care. For this let us again turn to the case of two instantons. At a generic point in the D-instanton moduli space, where they are at different locations, we have zero modes from the 11 open strings and 22 open strings, and we need to learn how to integrate over them. As usual we begin by leaving them 
unintegrated and treat them as external states of the diagram to construct the Wilsonian effective action. First consider the diagrams in the open closed string field theory where we have only type 1 and type 2 D-instanton vertices but no type 12 vertex or annulus. In this case we fuse all the 1 type vertices together and all the 2 type vertices together to construct a pair of composite vertices. Let us call them composite vertex 1 and 2 respectively. All the external 11 string zero modes will attach themselves to the composite vertex 1 , since in the original open closed string field theory they can only attach to type 1 or type 12 type vertices. Similarly all the external 22 string zero modes will attach themselves to the composite vertex 2 . As a result the integration over these zero modes become completely independent of each other and reduce to two copies of the integration procedure described in section 4.1. Therefore integration over these zero modes produce exactly the factors needed to make each composite vertex into a full 1-instanton vertex of the closed string effective field theory, including the momentum conserving delta functions associated with each composite vertex.

Next consider the case where the original Feynman diagram in the open closed string field theory has a 12 type vertex and / or a 12 type annulus. In this case there is only one composite vertex after we fuse all the D-instanton type vertices of the original diagram into a single composite vertex. Therefore both the 11 and 22 zero mode fields will attach to the same composite vertex and the integral over the zero modes no longer factors into two independent integrals. We can proceed as before. We now expect two ghost zero modes that, after field redefinition, represents the effect of division by the rigid $\mathrm{U}(1) \times \mathrm{U}(1)$ gauge transformation on the two D-instantons. The Jacobian associated with this field redefinition is no longer given by the product of two independent Jacobians associated with individual D-instantons and need to be recomputed. There are also a pair of auxiliary open string fields from the ghost sector whose contribution to the Feynman diagrams must be explicitly computed, producing additional contribution to the two instanton interaction term. Finally there are now two sets of zero modes, associated with the space-time coordinates of the D-instantons. After appropriate field redefinition, one of them, which we shall denote by $\phi$, will represent the average of the positions of the two D-instantons. The dependence on this mode will be proportional to $e^{i p . \phi}$ where $p$ is the total momentum carried by the closed strings connected to the composite vertex, and integration over $\phi$ gives the momentum conserving delta function at the 2-instanton interaction vertex of the closed string effective field theory. The other set of zero modes $\chi$ represent the difference between the locations of the two D-instantons after appropriate field redefinition. The amplitude will have complicated but computable dependence on $\chi$ and the integration over $\chi$ needs to be computed explicitly to find the 2 -instanton vertex of the effective field theory.

Since for large separation between the D-instantons the 12 open strings become very massive, the contribution to the interaction vertex of the closed string effective field theory from any Riemann surface that includes both a 1-type boundary and a 2-type boundary, is exponentially suppressed for large $\chi^{2}$. Therefore in these contributions, the $\chi$ integral does not have any divergence from the large $|\chi|$ region. This will be seen explicitly in an example in section 7 , where the function $f(\chi)$ in (7.16) has such exponential suppression factor. The full amplitude of course does receive contribution from the large $|\chi|$ region, but 
these come from regions of the moduli space associated with closed string degeneration, and are represented, in the open closed string field theory, by Feynman diagrams involving closed string exchanges. These are not part of the definition of the 2-instanton interaction vertex of the closed string effective field theory.

As we reduce $\chi^{2}$, the mass ${ }^{2}$ of some of the states of the 12 open string may change from being positive to being negative below a critical separation between the two D-instantons. These may lead to additional divergences that were not present in one instanton amplitudes. As we shall see in section 5.5, such divergences may be regulated by appropriate $i \epsilon$ prescription, but could lead to complex contribution to the closed string effective action.

The generalization of this analysis to the $k$ instanton case is straightforward. For any Feynman diagram of the original open closed string theory, once we fuse the interaction vertices into composite vertices following the rules mentioned earlier, for every composite vertex there will be a momentum conserving delta function from integration over the collective coordinates, consistent with the interpretation that every composite vertex is part of an interaction vertex of the closed string effective field theory. The new vertex that arises from these Feynman diagrams - the $k$ D-instanton vertex - will have a single momentum conserving delta function from integration over the collective coordinates that describes the average location of all the $k$ D-instantons. The dependence of the Wilsonian effective action on the other collective coordinates, describing the differences between the locations of the D-instantons, will be complicated but computable, and the $k$-instanton interaction vertex of the closed string effective field theory will include integration over these collective coordinates. As before, the integrand will be exponentially suppressed for large separation between the D-instantons, and there is no divergence in the integral from this region. However at finite separation, when some of the open strings connecting different D-instantons turn tachyonic, the integrand may have additional divergences that need to be dealt with by $i \epsilon$ prescription. This will be discussed in section 5.5.

\subsection{Identical D-instantons}

The case of $k$ identical D-instantons can be analyzed in more or less the same way since different D-instantons can still be distinguished by their locations in space-time. We shall of course get extra factor of $1 / k$ ! in the expression for the effective action since exchanging the locations of the instantons does not generate new configurations. The extra complication arises from the fact that in the limit when the instantons coincide, additional massless states appear. These could lead to new divergences from two sources. First, the $i j$ type annulus diagram receives a contribution proportional to $1 / \mathrm{m}^{2}$ from a pair of bosonic open string states of mass $m$ and a contribution proportional to $m^{2}$ from a pair of fermionic open string states of mass $m .^{7}$ These could diverge in the $m \rightarrow 0$ limit, but if there are more fermionic / ghost modes of this type than bosonic modes then these divergences could be avoided. The other possible source of divergences is from internal propagators of ij type open strings which could give $1 / \mathrm{m}^{2}$ factors. These divergences become more severe

\footnotetext{
${ }^{7}$ Since for every state of the open string stretched from the $i$-th instanton to the $j$-th instanton, there is a degenerate state of the open string stretched from the $j$-th instanton to the $i$-th instanton, the states come in pairs.
} 
at higher orders when there can be more $i j$ type open string propagators. To overcome these problems, we need to treat these zero modes also via Wilsonian effective action. This means that when the separation between the D-instantons go below some chosen cut-off, we explicitly remove the contribution of the new light modes from the internal propagators and regard them as background, and at the end explicitly perform the integrals over these new light modes. The final result should be independent of the precise choice of this cut-off.

As discussed in section 3.1, and elaborated in section 7.1, 7.2, the connection between the reality properties of the Euclidean and the Lorentzian action, that we are using in our analysis, relies on the effective action being an analytic function of the external energies in the complex plane. One could wonder if the extra massless modes on coincident Dinstantons could introduce non-analyticity of the effective action in the complex energy plane, thereby spoiling this connection. However, since derivatives with respect to external energy bring down powers of the separation $\chi$ between the instantons in the numerator of the integrand, and since the extra massless modes arise when the separations vanish, these derivatives do not make the integrand more singular. Therefore we do not expect the presence of these massless modes to introduce non-analyticities in the complex energy plane.

Finally, we would like to note that in special cases, massless open string modes may also appear on a pair of non-identical D-instantons when they coincide. The effect of these modes may be analyzed in the same way as described above.

\subsection{Gauge invariance}

We shall now verify the gauge invariance of the effective action. We shall first analyze the two instanton contribution to the closed string effective action, and then give a general argument for the gauge invariance of the multi-instanton contribution to the action.

As in section 4.3 , we shall begin with the result of [25], demonstrating the gauge invariance of the closed string effective action from connected world-sheet. For two identical instantons, these contributions may be of three types. First we have the contribution from world-sheet without boundaries. This was called $S_{0}$ in section 4.3. Then we have the contribution from the world-sheet with at least one boundary, with all the boundaries ending on the same D-instanton. This contribution is given by $2 S_{1}$, with $S_{1}$ being the same as what appears in (4.3). The factor of 2 is related to the fact that the boundaries may lie on either the first instanton or the second instanton. Finally we have the contribution from world-sheet with two or more boundaries, with at least one boundary lying on the first instanton and at least one boundary lying on the second instanton. We shall denote this contribution by $S_{2}$. Then $S_{0}+2 S_{1}+S_{2}$ will satisfy the BV master equations:

$$
\frac{1}{2}\left\{S_{0}+2 S_{1}+S_{2}, S_{0}+2 S_{1}+S_{2}\right\}+\Delta\left(S_{0}+2 S_{1}+S_{2}\right)=0 .
$$

Using (4.5) and (4.6), this can be reduced to

$$
\left\{S_{0}, S_{2}\right\}+2\left\{S_{1}, S_{2}\right\}+\left\{S_{1}, S_{1}\right\}+\frac{1}{2}\left\{S_{2}, S_{2}\right\}+\Delta S_{2}=0 .
$$

In the current problem, we include in the definition of the 2-instanton effective action the disconnected diagrams and also include a multiplicative factors of $K^{2}$ where $K=$ 
$\mathbf{N} e^{-C / g_{s}}$ as in section 4.3. Including the 0-instanton and 1-instanton contributions to the effective action introduced in section 4.3, the total effective action up to 2-instantons is given by,

$$
S_{\text {total }}=S_{0}+K\left(e^{S_{1}}-1\right)+\frac{1}{2} K^{2}\left(e^{S_{2}}-1\right) e^{2 S_{1}} .
$$

In the last term the factor of $e^{S_{2}}-1$ implies that the diagram must contain at least one connected component with both types of boundaries. As discussed in section 5.1, if such a component is absent, then the contribution to the amplitude may be regarded as the one coming from a pair of one instanton interaction vertices of the effective field theory, and does not represent a genuine 2-instanton contribution to the effective action. $e^{2 S_{1}}$ factor reflects that we can have arbitrary number of connected components whose boundaries all end on the first instanton and also an arbitrary number of connected components whose boundaries all end on the second D-instanton. The factor of $1 / 2$ reflects that the exchange of the two D-instantons does not generate a new configuration.

We now need to verify that $S_{\text {total }}$ satisfies the BV master equation to order $K^{2}$. We have already seen that it satisfies the master equation up to order $K$. Therefore we only have to examine the terms of order $K^{2}$. We have, to this order,

$$
\begin{aligned}
\frac{1}{2}\left\{S_{\text {total }}, S_{\text {total }}\right\}+\Delta S_{\text {total }}= & K^{2}\left[\frac{1}{2} e^{S_{2}+2 S_{1}}\left\{S_{0}, S_{2}\right\}+e^{2 S_{1}}\left(e^{S_{2}}-1\right)\left\{S_{0}, S_{1}\right\}+\frac{1}{2} e^{2 S_{1}}\left\{S_{1}, S_{1}\right\}\right. \\
& +\frac{1}{2} e^{S_{2}+2 S_{1}}\left(\Delta S_{2}+\frac{1}{2}\left\{S_{2}, S_{2}\right\}\right)+\left(e^{S_{2}}-1\right) e^{2 S_{1}}\left(\Delta S_{1}+\left\{S_{1}, S_{1}\right\}\right) \\
& \left.+e^{S_{2}+2 S_{1}}\left\{S_{2}, S_{1}\right\}\right]
\end{aligned}
$$

Using (4.6) and (5.5) one can show that the right hand side vanishes. Therefore we have:

$$
\frac{1}{2}\left\{S_{\text {total }}, S_{\text {total }}\right\}+\Delta S_{\text {total }}=0 .
$$

This proves gauge invariance of the closed string effective field theory up to two instanton order when the instantons are identical. The case where the instantons are not identical can be analyzed with minor modifications of the above argument. Let us denote by $S_{1}$ and $S_{1}^{\prime}$ the single instanton effective actions associated with connected world-sheet for the first and the second instanton respectively, by $K$ and $K^{\prime}$ the corresponding normalization factors, and by $S_{2}^{\prime}$ the analog of $S_{2}$ appearing in (5.4), except that the two instantons now refer to different instantons. Then in (5.4), $2 S_{1}$ will be replaced by $S_{1}+S_{1}^{\prime}, S_{2}$ will be replaced by $S_{2}^{\prime}$ and besides (4.6), we also have a similar equation involving $S_{1}^{\prime}$. Using this we can get the analog of (5.5):

$$
\left\{S_{0}, S_{2}^{\prime}\right\}+\left\{S_{1}, S_{2}^{\prime}\right\}+\left\{S_{1}^{\prime}, S_{2}^{\prime}\right\}+\left\{S_{1}, S_{1}^{\prime}\right\}+\frac{1}{2}\left\{S_{2}^{\prime}, S_{2}^{\prime}\right\}+\Delta S_{2}^{\prime}=0 .
$$

The analog of (5.6) now takes the form

$$
S_{\text {total }}=S_{0}+K\left(e^{S_{1}}-1\right)+K^{\prime}\left(e^{S_{1}^{\prime}}-1\right)+K K^{\prime}\left(e^{S_{2}^{\prime}}-1\right) e^{S_{1}+S_{1}^{\prime}},
$$


and our goal will be to show that the order $K K^{\prime}$ term in

$$
\frac{1}{2}\left\{S_{\text {total }}, S_{\text {total }}\right\}+\Delta S_{\text {total }}
$$

vanishes. This is given by:

$$
\begin{aligned}
K K^{\prime}[ & e^{S_{1}+S_{1}^{\prime}}\left\{S_{1}, S_{1}^{\prime}\right\}+e^{S_{1}+S_{1}^{\prime}+S_{2}^{\prime}}\left\{S_{0}, S_{2}^{\prime}\right\}+e^{S_{1}+S_{1}^{\prime}}\left(e^{S_{2}^{\prime}}-1\right)\left\{S_{0}, S_{1}+S_{1}^{\prime}\right\} \\
& +e^{S_{1}+S_{1}^{\prime}+S_{2}^{\prime}}\left(\Delta S_{2}^{\prime}+\frac{1}{2}\left\{S_{2}^{\prime}, S_{2}^{\prime}\right\}+\left\{S_{2}^{\prime}, S_{1}+S_{1}^{\prime}\right\}\right) \\
& \left.\quad+e^{S_{1}+S_{1}^{\prime}}\left(e^{S_{2}^{\prime}}-1\right)\left(\Delta S_{1}+\Delta S_{1}^{\prime}+\frac{1}{2}\left\{S_{1}, S_{1}\right\}+\frac{1}{2}\left\{S_{1}^{\prime}, S_{1}^{\prime}\right\}+\left\{S_{1}, S_{1}^{\prime}\right\}\right)\right] \\
= & 0
\end{aligned}
$$

where in the last step we have used (4.6), its analog with $S_{1}$ replaced by $S_{1}^{\prime}$ and (5.9).

We expect the gauge invariance of the effective action to hold also at higher instanton order, since the original amplitudes were gauge invariant, and our procedure for constructing the effective action simply organizes these amplitudes into Feynman diagrams of closed string effective field theory. The method described above becomes complicated due to complicated combinatorial factors. However, one can proceed following an alternate route in which one directly proves the main identities of [56], or equivalently the quantum $L_{\infty}$ algebra [61], for the interaction vertices of the closed string effective action constructed here. The key ingredient used in the proof in [56] is that the elementary interaction vertices, together with all other Feynman diagrams with propagators, cover the integration over the moduli spaces of Riemann surfaces in a one to one fashion. This property is also true by construction for the effective action described here, except that we include both connected and disconnected Riemann surfaces. This in turn would prove the gauge invariance of closed string field theory constructed here.

\subsection{Reality of the effective action}

The discussion of reality of the effective action proceeds as in the case of single instanton effective action. The main new ingredient is the integration over the collective coordinates labelling the differences between the D-instanton locations. The integrand is constructed from the Wilsonian effective action obtained by integrating out the massive and the tachyonic modes of the open string. The reality postulate of section 3.2 ensures that after factoring out the overall normalization constant $\mathbf{N}_{1} \cdots \mathbf{N}_{k}$, the integrand is invariant under simultaneous operation of complex conjugation, reversal of sign of external momenta, change of sign of the complex structure of the Riemann surface and change of sign of the collective coordinates. This would suggest that after integration over the moduli spaces of Riemann surfaces and the collective coordinates, we should obtain real contribution to the effective action, except for possible complex contribution from the $\mathbf{N}_{1} \cdots \mathbf{N}_{k}$ factor multiplying the $k$-instanton effective action.

We should not however forget the possibility that the apparent divergences from the boundaries of the moduli spaces associated with open string degeneration may lead to 
additional imaginary contribution. These can be analyzed more or less in the same way as in the single instanton case and can be shown not to introduce complex contribution, with one class of exceptions. As already discussed, when the separation between a pair of D-instantons passes through a critical value, some of the open string states connecting the pair may make transition from being massive to tachyonic. In such cases we need an $i \epsilon$ prescription to integrate through these singular points. These may lead to additional factors of $i$ that need to be accounted for.

We shall illustrate this by an example. Let us consider a two instanton system and let $\chi$ be the collective coordinate labelling the difference between the space-time locations of the pair of D-instantons. Let us further suppose that the spectrum of open strings stretched between the two D-instantons has a complex conjugate pair of states with mass ${ }^{2}$ given by

$$
\frac{\chi^{2}}{4 \pi^{2}}-M^{2}
$$

for some real constant $M$ so that for $|\chi|<2 \pi M$ the pair becomes tachyonic. The first term in (5.13) is the contribution due to the tension of the open string stretched over a distance $|\chi|$ in $\alpha^{\prime}=1$ unit, and the $-M^{2}$ term is the mass ${ }^{2}$ of the state for coincident D-instantons. Integrating out a pair of such open string modes will generate a factor in the amplitude proportional to

$$
\frac{4 \pi^{2}}{\chi^{2}-4 \pi^{2} M^{2}},
$$

from one loop determinant, i.e. the annulus diagram with the two boundaries lying on the two D-instantons. There may also be other singular factors, e.g. factors of $1 /\left(\chi^{2}-4 \pi^{2} M^{2}\right)$ from internal 12 string propagators. Now when we carry out the integration over $\chi$, the integral may no longer be real, since we need appropriate contour prescription around the point $\chi= \pm 2 \pi M$.

The divergence in (5.14) at $\chi=2 \pi M$ may appear to be unrelated to the divergences from the boundary of the moduli space, but this is not so. Indeed, the expression for the part of the amplitude given in (5.14), when expressed as integral over the moduli space of Riemann surfaces, has no divergence in the integrand at $\chi=2 \pi M$. Instead, what we have is an analog of (4.1) with $\alpha$ replaced by a term proportional to $\left(\chi^{2}-4 \pi^{2} M^{2}\right)$. As $\chi$ passes through $2 \pi M, \alpha$ passes through 0 , and although the integrand remain finite and real at fixed $q$, new divergences appear at $q=0$ for $\chi \leq 2 \pi M$, forcing us to adopt some contour prescription.

One possible choice of contour that was used in [12] is as follows. Since according to the general guideline discussed in section 3.1 the position space integration contour is rotated anti-clockwise as we go from the Euclidean to the Lorentzian theory, if we require that the $\chi$ integration contour does not cross a pole during this rotation, we need to choose the original poles to be below the real axis for $\chi^{0}>0$ and above the real axis for $\chi^{0}<0$. This can be summarized by saying that (5.14) should be replaced by [35]

$$
\frac{4 \pi^{2}}{\chi^{2}-4 \pi^{2} M^{2}+i \epsilon}
$$


The $i \epsilon$ generates an imaginary contribution to the Euclidean effective action after integration over $\chi$, which translates to an imaginary term in the Lorentzian effective action. We shall see an explicit example of this in section 7 . We shall call this procedure the Lorentzian contour prescription. In the multi-instanton amplitude there may be several singular factors of this type, and in the Lorentzian prescription we shall replace each such singular factor by a similar $i \epsilon$ prescription. This has been given in (1.3).

It is however not necessary to choose the contour using the prescription given in (5.15). An alternate prescription that does not generate an imaginary part of the interaction vertex of the closed string effective field theory and preserves unitarity is where we take the average of the Lorentzian prescription (5.15) and its complex conjugate, replacing all the $i \epsilon$ 's by $-i \epsilon$. We shall call this the unitary prescription. If we are to choose a uniform prescription that works for all string theories, then the unitary prescription seems more physical since a Lorentzian prescription will lead to unitarity violation also in critical superstring theory, e.g. from the D-instanton - anti-D-instanton contribution to type IIB string theory amplitudes. The actual computation is somewhat easier in the Lorentzian prescription since we can evaluate the integral over $\chi$ by rotating the integration contour to the Lorentzian space [12]. However once the result in the Lorentzian prescription has been obtained, the difference is easy to compute using the relation:

$$
\frac{1}{\chi^{2}-4 \pi^{2} M^{2}-i \epsilon}=\frac{1}{\chi^{2}-4 \pi^{2} M^{2}+i \epsilon}+2 i \pi \delta\left(\chi^{2}-4 \pi^{2} M^{2}\right) .
$$

We shall describe a simpler algorithm to compute the result in the unitary prescription at the end of section 7.1. The generalization of the unitary prescription at higher orders and / or for multiple instantons has been described below (1.3).

Ref. [12] used the Lorentzian prescription to evaluate the D-instanton contribution to the amplitudes of two dimensional string theory and found agreement with the matrix model results. We shall see in section 7 that at least in the 2-instanton and 3 -instanton sectors, the unitary prescription is also consistent with the matrix model results, with a different choice of the coefficients of the contribution from type $(2,1)$ and type $(3,1)$ ZZ-instantons.

In terms of the original integral over the tachyonic modes, the dependence on the contour prescription can be understood as follows. For $\chi>2 \pi M$ the tachyons have positive mass $^{2}$ and the steepest descent contours run along the real axes, while for $\chi<2 \pi M$ the tachyons have negative mass ${ }^{2}$ and the steepest descent contours run along the imaginary axes. This abrupt jump at $\chi=2 \pi M$ makes the choice of integration cycle ill defined. By deforming the $\chi$ integration contour around $2 \pi M$ into the complex plane, we ensure that the steepest descent contours for the tachyons rotate continuously from the real plane to the imaginary plane. However, now the combined integration cycle over $\chi$ and the tachyons depends on whether the $\chi$ contour passes below or above $2 \pi M$ in the complex plane, leading to different results for the integral.

In principle, however, there should be no ambiguity in carrying out the integral over the open string modes, at least in the critical superstring theory where the tachyon potential is bounded from below, with the minimum describing the perturbative vacuum [62]. Since 
a particular D-instanton configuration represents a saddle point of the Euclidean path integral, the integration over the open string modes need to be carried out along the steepest descent contours (Lefschetz thimbles [48-51, 63, 64]). The ambiguities we encounter here should be resolved once we express the original integration contour over the string fields as a union of the thimbles. At present this is not known since our understanding of the configuration space of string fields is limited. However, we shall make some guesses in section 8 .

In type IIB string theory, certain contribution to the effective action from the instanton - anti-instanton sector can be predicted [65-67]. It will be interesting to explore whether this can be reproduced using the techniques described above for constructing the multiinstanton contribution to the closed string effective action. I wish to thank Michael Green for raising this interesting question.

\subsection{Cutting rules}

We can now use the approach of [6] to prove the cutting rules in the closed string effective field theory, provided the effective action satisfies the reality condition. We can also translate these rules to the original Feynman diagrams of open closed string field theory as follows. Since the closed string effective field theory satisfies ordinary cutting rules, the cut does not pass through any interaction vertex. Therefore when we represent this as a cut diagram in the open closed string field theory, all the D-instanton labels of a given type must lie on one side of the cut. Let $U$ denote the set of labels carried by the interaction vertices on the left side of the cut and the complementary set $U^{c}$ denote the set of labels carried by the interaction vertices on the right side of the cut. Then the interaction vertices on the left of the cut are of type $\left(i_{1}, \cdots, i_{m}\right)$ with $i_{1}, \cdots, i_{m} \in U$ and the interaction vertices on the right of the cut are of type $\left(j_{1}, \cdots, j_{p}\right)$ with $j_{1}, \cdots, j_{p} \in U^{c}$. This is exactly the desired cutting rule discussed in section 2. Furthermore, since every internal open string propagator of the original Feynman diagrams is hidden inside the interaction vertices of the closed string effective field theory, an open string propagator is never cut, in agreement with the fact that open string states are not asymptotic states and should not appear as part of the states $|n\rangle$ in (2.1). The momentum conservation for diagrams on either side of the cut follows from the momentum conservation rules at the interaction vertices of closed string effective field theory. This allows us to identify the contributions from two sides of the cut as matrix elements of $T^{\dagger}$ and $T$ between closed string states.

\section{One instanton amplitudes in two dimensional string theory}

In this section we shall verify the results on one instanton amplitude, described in section 4 , in the context of two dimensional bosonic string theory.

\subsection{Leading one instanton contribution}

The world-sheet theory of the two dimensional string consists of a free scalar describing the time coordinate and a $c=25$ Liouville theory describing the space coordinate, besides the usual $b, c$ ghost system. The physical closed string spectrum of this theory has a single 
massless scalar field in two dimensions - called the tachyon. In this theory the leading Dinstanton contribution to the scattering amplitude for one incoming closed string tachyon of energy $\tilde{\omega}_{1}=-\omega_{1}>0$ and $(n-1)$ outgoing closed string tachyons of energies $\omega_{2}, \cdots, \omega_{n}>0$ was computed in [11] as the product of $n$ disk one point functions, with the result:

$$
T_{1-\text { inst }}=\mathbf{N} e^{-1 / g_{s}} \prod_{i=1}^{n}\left\{2 \sinh \left(\pi\left|\omega_{i}\right|\right)\right\} 2 \pi \delta\left(\sum_{i=1}^{n} \omega_{i}\right) .
$$

The result (6.1) is valid for arbitrary signs of $\omega_{i}$, with the understanding that negative $\omega_{i}$ denotes an incoming particle of energy $-\omega_{i}$. The value of the constant $\mathbf{N}$ will be described shortly.

We shall now write down an interaction term in the closed string effective action that produces the term in the amplitude given in (6.1). Clearly there is no unique off-shell continuation, but we shall write down the term that follows naturally from the open-closed string field theory. It takes the form:

$$
\frac{1}{n !} \mathbf{N} e^{-1 / g_{s}} \int \prod_{i=1}^{n}\left\{d \omega_{i} d P_{i} 2 \sinh \left(2 \pi P_{i}\right) \Phi_{C}\left(\omega_{i}, P_{i}\right) e^{-b\left(P_{i}^{2}-\omega_{i}^{2} / 4\right)}\right\} 2 \pi \delta\left(\sum_{j=1}^{n} \omega_{j}\right),
$$

where $\Phi_{C}(\omega, P)$ denotes the closed string tachyon field of energy $\omega$ and Liouville momentum $P$, in the convention in which the on-shell condition takes the form $P=|\omega| / 2 . b$ is a positive constant that is inherited from string field theory definition of disk one point function of off-shell external states,

$$
2 \sinh (2 \pi P) \exp \left[-b\left(P^{2}-\omega^{2} / 4\right)\right],
$$

and is known as the stub length. Note that $b$ has no effect on on-shell amplitudes, but acts as a damping term for off-shell Euclidean momenta, thereby making integration over internal momenta ultra-violet finite as long as the ends of the integration contour for internal energies are pinned at $\pm i \infty$ [6]. The overall normalization of (6.2) follows from (6.1) and our discussion in section 3.1, In particular, since (6.1) gives the expression for the T-matrix, there is no extra factor of $i$ or -1 in relating the normalization constants in (6.1) and (6.2).

The normalization constant $\mathbf{N}$ was computed in [11] from comparison of the result with the matrix model, yielding the result:

$$
\mathbf{N}=\frac{i}{8 \pi^{2}}
$$

Note that since [11] stated the result for the S-matrix, they had a normalization constant $-1 /\left(8 \pi^{2}\right)$. Since in our convention the amplitude refers to the T-matrix, we have the factor of $i$ in the expression for $\mathbf{N}$. The presence of $i$ in (6.4) shows that the single D-instanton terms in the effective action are imaginary. It follows from our results in section 4 that this will be the sole source of unitarity violation for all single D-instanton induced amplitudes in two dimensional bosonic string theory.

One could ask if it is possible to see the factor of $i$ directly in the string theory analysis. A tentative answer is that this is the result of integration over the open string tachyon on the 
D-instanton. ${ }^{8}$ If we denote the open string tachyon field by $T$ and it mass ${ }^{2}$ by $-M^{2}$, then the integration over the tachyon mode in the Euclidean path integral has to be performed by taking the integration contour to lie along the imaginary axis, and generates a factor,

$$
\int_{\mp i \infty}^{ \pm i \infty} d T e^{M^{2} T^{2}}= \pm i \frac{\sqrt{\pi}}{M}
$$

This factor of $i$ agrees with the one appearing in (6.1). However since we do not yet have a full derivation of $\mathbf{N}$ in string theory, this is not a definitive conclusion. It will be interesting to find a definitive criteria for determining the (lack of) reality of $\mathbf{N}$ for some given D-instanton in a given string theory, since a complex $\mathbf{N}$ would signal transition of closed strings to other (D-brane) states in the theory. ${ }^{9}$

Since for real $\mathbf{N}$ the term (6.2) is real and there is no violation of unitarity, we are led to the conclusion that just by studying single D-instanton amplitude in two dimensional string theory with the current level of understanding, we cannot come to a definitive conclusion that the theory is non-unitary - we need comparison with the matrix model to determine that $\mathbf{N}$ is imaginary. We shall test this in section 6.2 by showing that the amplitudes satisfy cutting rules for real $\mathbf{N}$. In section 6.3 we shall discuss the effect of having complex $\mathbf{N}$.

\subsection{Unitarity of one instanton amplitude for real $\mathrm{N}$}

Within the context of two dimensional string field theory, assuming $\mathbf{N}$ to be real does not violate any of the basic principles - at least at the level we are analyzing the theory. In order to illustrate the point that the violation of unitarity in two dimensional string theory arises from the factor of $i$ in $\mathbf{N}$, we shall now show how the one instanton contribution would have satisfied the correct cutting rules if $\mathbf{N}$ had been real. This will be based largely on the analysis of $[36,68]$, but for the sake of completeness we have included it here with the new viewpoint and appropriate factors of $i$.

We shall consider the case of a two point closed string tachyon amplitude, with the incoming tachyon of energy $\tilde{\omega}_{1}=-\omega_{1}>0$ and an outgoing tachyon of energy $\omega_{2}>0$. We shall focus on a particular world-sheet contribution that involves the product of a disk one point function for the outgoing closed string tachyon of energy $\omega_{2}$ and annulus one point function for the incoming closed string tachyon of energy $\tilde{\omega}_{1}$. The divergent part of this amplitude associated with closed string degeneration is given by [11]:

$$
\begin{aligned}
& 2 \pi \delta\left(\tilde{\omega}_{1}-\omega_{2}\right) \mathbf{N} e^{-1 / g_{s}} g_{s} \sinh \left(\pi \omega_{2}\right) 2^{11 / 2} \pi \int_{0}^{\infty} d P_{1} \int_{0}^{\infty} d P_{2} \mathcal{C}\left(\tilde{\omega}_{1} / 2, P_{1}, P_{2}\right) \\
& \sinh \left(2 \pi P_{1}\right) \sinh \left(2 \pi P_{2}\right) \int_{c}^{\infty} d s \int_{0}^{\frac{1}{4}} d x s^{1 / 2} \exp \left[-2 \pi s\left\{(1-2 x) P_{1}^{2}+2 x P_{2}^{2}-x\left(\frac{1}{2}-x\right) \tilde{\omega}_{1}^{2}\right\}\right],
\end{aligned}
$$

where the lower limit $c$ of $s$ integration is some positive number and $\mathcal{C}\left(P_{1}, P_{2}, P_{3}\right)$ is the genus zero three point function of three Liouville primaries carrying momenta $P_{1}, P_{2}$ and

\footnotetext{
${ }^{8}$ This is a somewhat different point of view from the one given in footnote 2 of [11].

${ }^{9}$ Since $\mathbf{N}$ reflects the ratio of integration measures around the D-instanton and the perturbative vacuum, one could in principle compute $\mathbf{N}$ by regarding the $\mathrm{D}$-instanton and the perturbative vacuum as different classical solutions of the same underlying open closed string field theory.
} 
$P_{3}[69,70]$. The factor of $1 / 2$ in the first argument of $\mathcal{C}$ arises due to the fact that in the normalization convention of [47], which we are using, the on-shell condition for a closed string tachyon of energy $\omega$ and momentum $P$ is $\omega=2 P$. As discussed in section 4 , like all string theory amplitudes this amplitude is formally real ${ }^{10}$ for real $\mathbf{N}$ and the possible imaginary parts would have to arise from the process of making sense of the divergences. In this case the relevant divergences arise from the $s \rightarrow \infty$ limit. By making change of variables

$$
t_{1}=2 \pi s(1-2 x), \quad t_{2}=4 \pi s x,
$$

and symmetrizing the integral under $t_{1} \leftrightarrow t_{2}, P_{1} \leftrightarrow P_{2}$ exchange, we can rewrite this as [68]

$$
\begin{aligned}
& 2 \pi \delta\left(\tilde{\omega}_{1}-\omega_{2}\right) 4 \mathbf{N} e^{-1 / g_{s}} g_{s} \sinh \left(\pi \omega_{2}\right) \pi^{-1 / 2} \int_{0}^{\infty} d P_{1} \int_{0}^{\infty} d P_{2} \mathcal{C}\left(\tilde{\omega}_{1} / 2, P_{1}, P_{2}\right) \\
& \sinh \left(2 \pi P_{1}\right) \sinh \left(2 \pi P_{2}\right) \int_{2 \pi c \leq t_{1}+t_{2}<\infty} d t_{1} d t_{2}\left(t_{1}+t_{2}\right)^{-1 / 2} \exp \left[-t_{1} P_{1}^{2}-t_{2} P_{2}^{2}+\frac{t_{1} t_{2}}{t_{1}+t_{2}} \frac{\tilde{\omega}_{1}^{2}}{4}\right] .
\end{aligned}
$$

Now it is easy to see that for $\tilde{\omega}_{1} \geq 2\left(P_{1}+P_{2}\right)$, the integration over $t_{1}, t_{2}$ diverges for $t_{1}, t_{2} \rightarrow \infty$, but there is no divergence when any one of them remains finite even if the other one approaches $\infty$. Using this, we can express the divergent part of (6.8) as:

$$
\begin{aligned}
& 2 \pi \delta\left(\tilde{\omega}_{1}-\omega_{2}\right) 4 \mathbf{N} e^{-1 / g_{s}} g_{s} \sinh \left(\pi \omega_{2}\right) \pi^{-1 / 2} \int_{0}^{\infty} d P_{1} \int_{0}^{\infty} d P_{2} \mathcal{C}\left(\tilde{\omega}_{1} / 2, P_{1}, P_{2}\right) \\
& \sinh \left(2 \pi P_{1}\right) \sinh \left(2 \pi P_{2}\right) \int_{b}^{\infty} d t_{1} \int_{b}^{\infty} d t_{2}\left(t_{1}+t_{2}\right)^{-1 / 2} \exp \left[-t_{1} P_{1}^{2}-t_{2} P_{2}^{2}+\frac{t_{1} t_{2}}{t_{1}+t_{2}} \frac{\tilde{\omega}_{1}^{2}}{4}\right]
\end{aligned}
$$

for some positive constant $b$. The difference between (6.9) and (6.8) is real and finite for real $\mathbf{N}$ and will not be discussed further. Using the results of [71], (6.9) can be rewritten as:

$$
\begin{gathered}
-\frac{1}{2} i \mathbf{N} e^{-1 / g_{s}} g_{s} 2 \sinh \left(\pi \omega_{2}\right) \int_{0}^{\infty} d P_{1} \int_{0}^{\infty} d P_{2} C\left(\tilde{\omega}_{1} / 2, P_{1}, P_{2}\right) 2 \sinh \left(2 \pi P_{1}\right) 2 \sinh \left(2 \pi P_{2}\right) \\
\int \frac{d \widetilde{\omega}}{2 \pi} \frac{1}{-\frac{1}{4}\left(\tilde{\omega}_{1}-\widetilde{\omega}\right)^{2}+P_{1}^{2}-i \epsilon} \frac{1}{-\frac{1}{4} \widetilde{\omega}^{2}+P_{2}^{2}-i \epsilon} \exp \left[-b\left(P_{1}^{2}+P_{2}^{2}\right)+\frac{b}{4} \widetilde{\omega}^{2}+\frac{b}{4}\left(\tilde{\omega}_{1}-\widetilde{\omega}\right)^{2}\right] \\
\times 2 \pi \delta\left(\tilde{\omega}_{1}-\omega_{2}\right) .
\end{gathered}
$$

As shown in figure 5, the $\widetilde{\omega}$ integration contour runs along the real axis in the region where the poles are situated, but then returns to $\pm i \infty$ by remaining at large distances in the first and the third quadrant. This makes the integral finite due to the exponential suppression factor. Note that the equality of (6.9) and (6.10) is an identity for $\tilde{\omega}_{1}^{2}<0$, which can be proved by using the Schwinger parameter representation for the two propagators in (6.10)

\footnotetext{
${ }^{10}$ The relevant test is not reality but the hermiticity of an amplitude, which requires us to test invariance under complex conjugation, together with exchange of the incoming and the outgoing states, or equivalently change of the signs of all external momenta in the convention that an incoming particle of momentum $p$ is regarded as an outgoing particle of momentum $-p$. In (6.6) the exchange of the incoming and the outgoing states simply exchanges $\tilde{\omega}_{1}$ and $\omega_{2}$. This has no effect since $\tilde{\omega}_{1}=\omega_{2}$ after using energy conservation.
} 


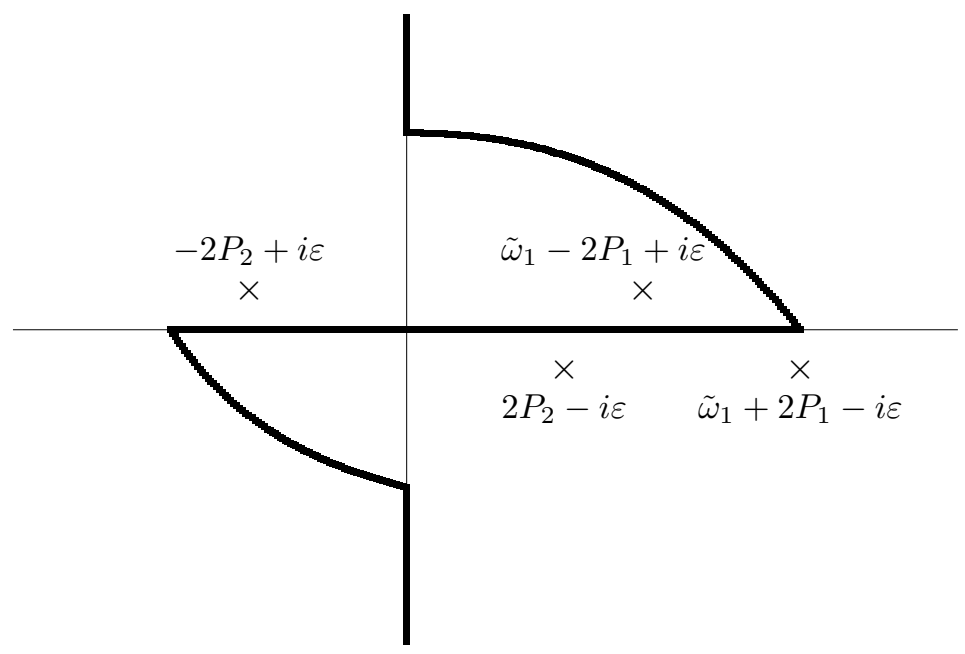

Figure 5. The thick contour in this figure shows the choice of $\tilde{\omega}$ integration contour in (6.10). The $\times$ 's mark the locations of the poles in the complex $\tilde{\omega}$ plane.

and then carrying out the integration over $\widetilde{\omega}$ by deforming the integration contour to lie along the imaginary axis. For $\tilde{\omega}_{1}^{2}>0,(6.9)$ diverges due to the divergence in the $t_{i}$ integral for large $t_{1}, t_{2}$ but (6.10) is finite. Therefore (6.10) is the analytic continuation of (6.9) for $\tilde{\omega}_{1}^{2}>0$.

We can now compute the imaginary part of (6.10) by deforming the $\widetilde{\omega}$ integration contour to lie along the imaginary axis, and picking up the residue from the pole at $\tilde{\omega}_{1}-$ $2 P_{1}+i \varepsilon$ that it crosses on the way. The integral along the imaginary axis gives real result, since the effect of complex conjugation can be compensated by a change in the sign of the integration variable. The residue at the pole however gives a complex contribution to the final result. The final result for $2 \times$ the imaginary part, assuming that $\mathbf{N}$ is real, is given by [68]:

$$
\begin{gathered}
2 i \mathbf{N} e^{-1 / g_{s}} g_{s} 2 \sinh \left(\pi \omega_{2}\right) \int_{0}^{\infty} d \omega_{3} \int_{0}^{\infty} d \omega_{4} \mathcal{C}\left(\tilde{\omega}_{1} / 2, \omega_{3} / 2, \omega_{4} / 2\right) 2 \pi \delta\left(\omega_{3}+\omega_{4}-\tilde{\omega}_{1}\right) \\
\left(2 \omega_{3}\right)^{-1}\left(2 \omega_{4}\right)^{-1}\left\{2 \sinh \left(\pi \omega_{3}\right)\right\}\left\{2 \sinh \left(\pi \omega_{4}\right)\right\} 2 \pi \delta\left(\tilde{\omega}_{1}-\omega_{2}\right) .
\end{gathered}
$$

We shall now give the Feynman diagram interpretation of (6.10) and (6.11) in the open closed string field theory. (6.10) can be interpreted as the contribution from the Feynman diagram shown in figure $6(\mathrm{a})$. The $\times$ denotes a disk one point function and produces the factors of $2 \sinh (2 \pi P) \exp \left[-b\left(P^{2}-\omega^{2} / 4\right)\right]$, given in (6.3), from the one point function of the Liouville primary of momentum $P$ and energy $\omega$ on the disk. $\omega_{2}$ and $\tilde{\omega}_{1}$ label the outgoing and incoming energies of the original Feynman diagram and $\omega_{3}=\tilde{\omega}_{1}-\widetilde{\omega}$ and $\omega_{4}=\widetilde{\omega}$ label internal energies, all flowing from left to right. $C\left(\tilde{\omega}_{1} / 2, P_{1}, P_{2}\right)$ represents the three point interaction vertex of three closed strings. For off-shell external states, we could include additional damping factors in this vertex by replacing $b$ by $b^{\prime}>b$ in (6.9) and (6.10), but have chosen not to do so. The two factors in the second line of (6.10) represent the propagators of the internal lines in figure $6\left(\right.$ a), carrying energies $\omega_{3}=\tilde{\omega}_{1}-\widetilde{\omega}$ and $\omega_{4}=\widetilde{\omega}$. Integration over the zero mode $\phi$, labelling the position of the D-instanton 


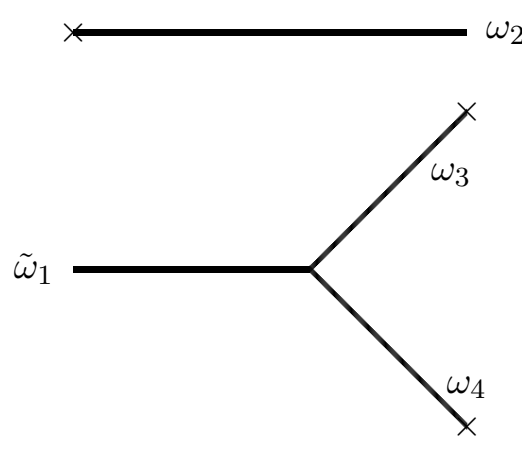

(a)

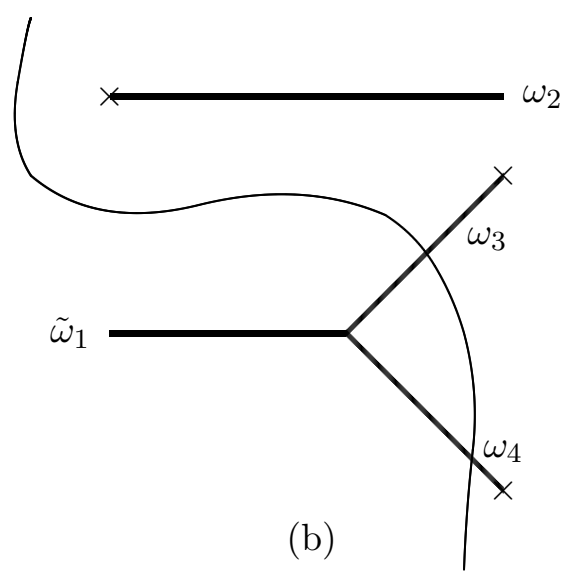

(b)

Figure 6. The figure on the left shows a Feynman diagram of open closed string field theory, describing part of the contribution to the product of an annulus one point function and a disk one point function. $\mathrm{A} \times$ denotes the one point closed string interaction vertex associated with one point function on the disk and a free end marks an external state. All energies flow from the left to the right. The figure on the right is a cut of this diagram, with the thin curve denoting the cut, drawn such that all the D-instanton type interaction vertices are to the right of the cut. The corresponding Feynman diagrams in the closed string effective field theory are obtained by collapsing all the $\times$ 's into a single composite vertex. These have been shown in figure 7 .

along the Euclidean time, produces the energy conserving delta function $2 \pi \delta\left(\tilde{\omega}_{1}-\omega_{2}\right)$ after Wick rotation. The factors of $i$ can be understood as follows. If we were computing the S-matrix element, then we would get factor of $-i$ from each of the two propagators, a factor of $i$ from the closed string three point vertex and a factor of $i$ from the D-instanton induced vertex of the effective field theory, obtained by merging the three $\times$ in the diagram. The product of these factors give 1 . Note that we do not have separate $i$ from each $\times-$ this is part of the Feynman rule of the theory since we integrate out the open string zero modes in the Euclidean space where there are no $i$ 's in the interaction vertices and then rotate the effective field theory action to the Lorentzian space. To compute the T-matrix element we need to multiply the result by a factor of $-i$. This gives the overall multiplicative factor of $-i$ in (6.10). The factor of $1 / 2$ in the front is a combinatorial factor due to having two identical internal lines carrying energies $\omega_{3}$ and $\omega_{4}$. This may be clearer from figure $7(\mathrm{a})$ that gives the representation of the same term as a Feynman diagram in the closed string effective field theory.

Let us now turn to (6.11) and show that for real $\mathbf{N}$, it can be interpreted as the contribution to $T-T^{\dagger}$ from the cut diagram shown in figure 6(b) in accordance with (2.1). According to the cutting rule, to compute the difference between (6.10) and its hermitian conjugate, we simply have to replace the propagators carrying energies $\omega_{3}$ and $\omega_{4}$ by cut propagators,

$$
-\frac{i}{-\frac{1}{4} \omega_{i}^{2}+P^{2}-i \epsilon} \Rightarrow 2 \pi \delta\left(-\frac{1}{4} \omega_{i}^{2}+P^{2}\right), \quad i=3,4,
$$

and take hermitian conjugate of the diagram on the right of the cut. Since for real $\mathbf{N}$ the 


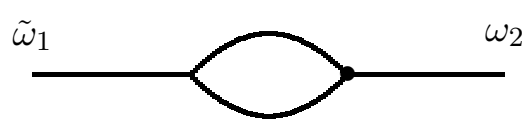

(a)

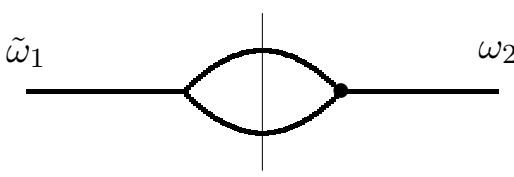

(b)

Figure 7. The figure shows the representations of figure 6(a) and (b) as Feynman diagrams of closed string effective field theory. • denotes a D-instanton type interaction vertex of the effective field theory.

diagram on the right of the cut is hermitian, taking its hermitian conjugate has no effect. This gives the contribution to $T-T^{\dagger}$ according to cutting rules to be:

$$
\begin{gathered}
-\frac{i}{2} \mathbf{N} e^{-1 / g_{s}} g_{s} 2 \sinh \left(\pi \omega_{2}\right) \int_{0}^{\infty} d P_{1} \int_{0}^{\infty} d P_{2} C\left(\omega / 2, P_{1}, P_{2}\right) 2 \sinh \left(2 \pi P_{1}\right) 2 \sinh \left(2 \pi P_{2}\right) \\
\int \frac{d \widetilde{\omega}}{2 \pi} 2 \pi i \delta\left(-\frac{1}{4}\left(\tilde{\omega}_{1}-\widetilde{\omega}\right)^{2}+P_{1}^{2}\right) 2 \pi i \delta\left(-\frac{1}{4} \widetilde{\omega}^{2}+P_{2}^{2}\right) 2 \pi \delta\left(\tilde{\omega}_{1}-\omega_{2}\right) .
\end{gathered}
$$

It is straightforward to verify that this precisely reproduces (6.11). Physically the cut diagram is given by the product of a genus zero contribution to closed string $1 \rightarrow 2$ amplitude and the hermitian conjugate of the leading D-instanton contribution to the closed string $1 \rightarrow 2$ amplitude, given by the product of three disk one point functions. Figure $7(\mathrm{~b})$ shows the representation of the same cut diagram in the closed string effective field theory.

\subsection{Unitarity violation in the matrix model}

We now turn to the case when $\mathbf{N}$ is imaginary as given in (6.4), and explore whether the analog of (2.1) can still give us some useful information. For this let us first rewrite (2.1) in a way that will be convenient to generalize to the case when $\mathbf{N}$ is not real. Let $T_{k}$ denote the contribution to $T$ from the $k$ instanton sector, containing terms proportional to $e^{-k / g_{s}}$. Then for hermitian $T$, comparing terms proportional to $e^{-1 / g_{s}}$ on two sides of (2.1), we get:

$$
\left\langle f\left|T_{1}\right| i\right\rangle-\left\langle f\left|T_{1}^{\dagger}\right| i\right\rangle=i \sum_{n}\left\langle f\left|T_{1}^{\dagger}\right| n\right\rangle\left\langle n\left|T_{0}\right| i\right\rangle+i \sum_{n}\left\langle f\left|T_{0}^{\dagger}\right| n\right\rangle\left\langle n\left|T_{1}\right| i\right\rangle .
$$

Now when $\mathbf{N}$ is imaginary, then we should expect an analog of (6.14) with $T_{1}$ replaced by $i T_{1}$ to hold, since up to order $e^{-1 / g_{s}}, T_{0}+i T_{1}$ will represent a T-matrix constructed from real effective action in which $\mathbf{N}$ has been replaced by $i \mathbf{N}$. Therefore we now expect a relation of the form:

$$
\left\langle f\left|i T_{1}\right| i\right\rangle-\left\langle f\left|\left(i T_{1}\right)^{\dagger}\right| i\right\rangle=i \sum_{n}\left\langle f\left|\left(i T_{1}\right)^{\dagger}\right| n\right\rangle\left\langle n\left|T_{0}\right| i\right\rangle+i \sum_{n}\left\langle f\left|T_{0}^{\dagger}\right| n\right\rangle\left\langle n\left|\left(i T_{1}\right)\right| i\right\rangle .
$$

Due to the extra factors of $i$, this relation is no longer useful for proving unitarity of the closed string amplitudes. Nevertheless, as we shall discuss now, this identity can still be used to extract useful information on T-matrix elements. 
The first term on the right hand side of (6.15) represents the contribution from the cut diagram figure 6(a) with $\mathbf{N}$ replaced by $i \mathbf{N}$. The second term on the right hand side represents the contribution from another diagram, obtained by mirror reflecting figure 6 about the vertical axis and exchanging $\omega_{2}$ and $\tilde{\omega}_{1}$. This gives the same contribution as the first term. Therefore the right hand side of (6.15) is given by twice the term given in (6.11), with $\mathbf{N}$ replaced by $i \mathbf{N}$. After cancelling the $i$ from two sides of (6.15), we get the matrix element of $T_{1}+T_{1}^{\dagger}$ for $1 \rightarrow 1$ scattering:

$$
\begin{gathered}
\left(T_{1}+T_{1}^{\dagger}\right)=2 \pi \delta\left(\tilde{\omega}_{1}-\omega_{2}\right) 4 i \mathbf{N} e^{-1 / g_{s}} g_{s} 2 \sinh \left(\pi \omega_{2}\right) \int_{0}^{\infty} d \omega_{3} \int_{0}^{\infty} d \omega_{4} \mathcal{C}\left(\tilde{\omega}_{1} / 2, \omega_{3} / 2, \omega_{4} / 2\right) \\
2 \pi \delta\left(\omega_{3}+\omega_{4}-\tilde{\omega}_{1}\right)\left(2 \omega_{3}\right)^{-1}\left(2 \omega_{4}\right)^{-1}\left\{2 \sinh \left(\pi \omega_{3}\right)\right\}\left\{2 \sinh \left(\pi \omega_{4}\right)\right\} \\
=2 \pi \delta\left(\tilde{\omega}_{1}-\omega_{2}\right) i \mathbf{N} e^{-1 / g_{s}} g_{s} 2 \sinh \left(\pi \omega_{2}\right) \int_{0}^{\infty} d \omega_{3} \int_{0}^{\infty} d \omega_{4} \tilde{\omega}_{1} 2 \pi \delta\left(\omega_{3}+\omega_{4}-\tilde{\omega}_{1}\right) \\
\left\{2 \sinh \left(\pi \omega_{3}\right)\right\}\left\{2 \sinh \left(\pi \omega_{4}\right)\right\},
\end{gathered}
$$

where we used $\mathcal{C}\left(\tilde{\omega}_{1} / 2, \omega_{3} / 2, \omega_{4} / 2\right)=\tilde{\omega}_{1} \omega_{3} \omega_{4}$ when $\tilde{\omega}_{1}=\omega_{3}+\omega_{4}$ [47]. Using $S=i T$, we now see that the matrix element of $S_{1}-S_{1}^{\dagger}$ for $1 \rightarrow 1$ scattering is given by:

$$
\begin{aligned}
\left(S_{1}-S_{1}^{\dagger}\right)= & -2 \pi \delta\left(\tilde{\omega}_{1}-\omega_{2}\right) \mathbf{N} e^{-1 / g_{s}} g_{s} 2 \sinh \left(\pi \omega_{2}\right) \int_{0}^{\infty} d \omega_{3} \int_{0}^{\infty} d \omega_{4} \tilde{\omega}_{1} 2 \pi \delta\left(\omega_{3}+\omega_{4}-\tilde{\omega}_{1}\right) \\
& \left\{2 \sinh \left(\pi \omega_{3}\right)\right\}\left\{2 \sinh \left(\pi \omega_{4}\right)\right\} \\
= & -2 \frac{i}{\pi} \delta\left(\tilde{\omega}_{1}-\omega_{2}\right) e^{-1 / g_{s}} g_{s} \tilde{\omega}_{1} \sinh ^{2}\left(\pi \tilde{\omega}_{1}\right)\left\{\frac{\pi \tilde{\omega}_{1}}{\tanh \left(\pi \tilde{\omega}_{1}\right)}-1\right\}
\end{aligned}
$$

where in the last step we have used (6.4) and explicitly carried out the integration over $\omega_{3}$ and $\omega_{4}$. This agrees with the dual matrix model result given in [11]. Similar agreement has been found for all $1 \rightarrow n$ scattering amplitudes [68].

\section{Two instanton amplitudes in two dimensional string theory}

In this section we shall examine the source of unitarity violation in 2-instanton amplitudes in two dimensional string theory and show that the results are consistent with the general conclusions of section 5. In section 7.1 we shall follow [12] and evaluate the leading 2instanton contribution to the two tachyon amplitude in two dimensional string theory using the Lorentzian prescription for the integration contour. However we shall not assume the value of $\mathbf{N}$, allowing it to be an arbitrary complex number. The amplitude will turn out to have an imaginary part even for real $\mathbf{N}$. In section 7.2 we shall analyze whether this imaginary part is consistent with the cutting rules. For this we analyze the same amplitude as a sum of Feynman diagrams in open closed string field theory, as well as in the closed string effective field theory. We shall find that while a part of the imaginary contribution can be traced to the contribution from a cut diagram, the rest of the contribution to the imaginary part cannot be regarded as coming from a cut diagram. However the difference can be accounted for by the contribution from the imaginary part of the closed string effective action originating in the 2-instanton sector. This shows violation of unitarity in the theory even when $\mathbf{N}$ is real. Furthermore, the imaginary part of the 2-instanton 
effective action can be traced directly to the $i \epsilon$ in the Lorentzian prescription, needed for dealing with the extra open string tachyon modes that appear on the D-instanton system when the two D-instantons come close. In section 7.3 we show that if we had used the unitary prescription for evaluating the amplitude then for real $\mathbf{N}$, the imaginary part of the amplitude would have been consistent with the cutting rules, thereby showing that we get a unitary amplitude. We also show that in this case the two instanton contribution to the closed string effective action is real. In section 7.4 we compare our conventions with those in [12], and verify that what we call the Lorentzian prescription indeed agrees with that given in [12] when we put all the factors of $i$ in the proper places and choose $\mathbf{N}$ to be $i /\left(8 \pi^{2}\right)$. We then show that if we had used the unitary prescription for computing the two instanton contribution in this theory, the agreement between the string theory results and the matrix model results found in [12] continues to hold, provided we choose a different normalization constant for the $(2,1)$ instanton amplitude. In section 7.5 we extend this analysis to the three instanton sector.

Before we go into the details of the analysis, one comment is in order. As discussed in section 5.3, when the instantons coincide, there are extra massless modes and new branches of the moduli space. For $k$ coincident instantons there will be $k^{2}-1$ such additional massless modes, related by $\mathrm{SU}(k)$ rotation to the modes that separate the D-instantons along the Euclidean time direction. Therefore below a cut-off distance, we should 'integrate in' the light open string modes, construct the Wilsonian effective action involving these light modes and then explicitly integrate the exponential of the effective action over these modes. However in the two dimensional string theory one can use the $\mathrm{SU}(k)$ symmetry to make the corresponding Chan-Paton matrix diagonal and therefore the integral reduces to the branch that we have been integrating over. Since this gauge is different from the Siegel gauge the integrand will look different, but since away from the coincident limit both gauge choices are legal, it is guaranteed that at the cut-off the integrand inside and outside match up after integrating out all massive modes. The residual gauge symmetry after gauge fixing includes the permutation group of the $k$ instantons and leads to the symmetry factor of $1 / k$ ! that was discussed in section 5.3.

\subsection{Leading two instanton contribution to the closed string two point function}

In this subsection we shall compute the leading two instanton contribution to the two point function of closed string tachyon following [12], with an incoming closed string of energy $\tilde{\omega}_{1}=-\omega_{1}>0$ and an outgoing closed string of energy $\omega_{2}>0$. In order to understand the analytic properties of various results as function of $\omega_{1}, \omega_{2}$, we shall express our result in a way that will be valid for both signs of the $\omega_{i}$ 's. There will be an overall normalization factor of $\mathbf{N}^{2} e^{-2 / g_{s}}$, with the $\mathbf{N}^{2}$ factor containing the effect of annulus amplitude with both boundaries lying on the same D-instanton. However we need to also include the effect of the annulus with the two boundaries lying on different D-instantons. This is given by [12]:

$$
\mathcal{A}_{12}=\int_{0}^{\infty} d t t^{-1}\left[e^{-t\left\{\left(\phi_{(1)}-\phi_{(2)}\right)^{2}-4 \pi^{2}\right\} / 2 \pi}-e^{-t\left\{\phi_{(1)}-\phi_{(2)}\right\}^{2} / 2 \pi}\right]=\ln \frac{\left\{\phi_{(1)}-\phi_{(2)}\right\}^{2}}{\left\{\phi_{(1)}-\phi_{(2)}\right\}^{2}-4 \pi^{2}} .
$$




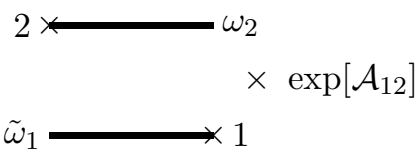

(a)

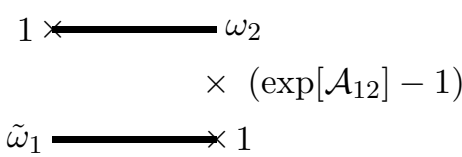

(b)

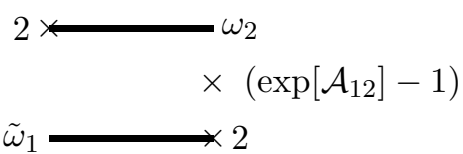

(c)

Figure 8. The leading order two instanton contribution to the two point function of closed strings. As usual $\times$ denotes the disk one point function. $\mathcal{A}_{12}$ denotes the full annulus contribution with the two boundaries lying on two different instantons. We also have a diagram in which 1 and 2 are exchanged in figure(a). The -1 in the last two terms ensures that the underlying world-sheet has at least one boundary of each type.

This includes the contribution to the partition function from open strings stretching from instanton 1 to instanton 2 and from open strings stretching from instanton 2 to instanton 1 - otherwise there would have been a factor of $1 / 2$ multiplying this expression. Note the subtle difference betwwen $\mathcal{A}_{12}$ and $A_{12}$ defined in (5.1). In the latter, the lower limit of integration is a constant $a>0$ since we subtract the contribution given by exchange of closed strings between a pair of disks in the underlying open closed string field theory. In contrast $\mathcal{A}_{12}$ represents the full annulus contribution. $\mathcal{A}_{12}$ is the relevant contribution while computing the full amplitude, whereas $A_{12}$ is the relevant contribution for computing the interaction vertex of the closed string effective field theory.

The leading 2-instanton contribution to the closed string two point function will come from the product of $\mathbf{N}^{2} e^{-2 / g_{s}} \exp \left[\mathcal{A}_{12}\right]$ times the product of two disk one point functions. However, if the boundaries of both the disks lie on the same D-instanton, then the multiplicative factor is $\mathbf{N}^{2} e^{-2 / g_{s}}\left(\exp \left[\mathcal{A}_{12}\right]-1\right)$, so that after expanding the exponential, each term represents a world-sheet diagram that has each type of boundary appearing at least once. These contributions have been shown in figure 8. Even though they look like Feynman diagrams of open closed string field theory, they should be interpreted as the full world-sheet contribution to the amplitude since we use $\mathcal{A}_{12}$ instead of $A_{12}$ in the exponent. For example, figure 8(a) represents an amplitude where the incoming closed string vertex operator is inserted at the center of the disk whose boundary lies on instanton 1 and the outgoing closed string vertex operator is inserted at the center of the disk whose boundary lies on instanton 2 . This amplitude is multiplied by $\exp \left[\mathcal{A}_{12}\right]$. There is a similar diagram (not shown in the figure) where the boundary conditions of the two disks are interchanged. They give identical contributions and their total contribution to the amplitude in the Euclidean theory is given by:

$$
\begin{aligned}
& \mathbf{N}^{2} e^{-2 / g_{s}} \int d \phi_{(1)} d \phi_{(2)} e^{-i \omega_{1}^{E} \phi_{(1)}-i \omega_{2}^{E} \phi_{(2)}} 2 \sinh \left(2 \pi P_{1}\right) 2 \sinh \left(2 \pi P_{2}\right) \exp \left[\mathcal{A}_{12}\right] \\
& =\mathbf{N}^{2} e^{-2 / g_{s}} 4 \sinh \left(2 \pi P_{1}\right) \sinh \left(2 \pi P_{2}\right) \int d \phi_{(1)} d \phi_{(2)} e^{-i \omega_{1}^{E} \phi_{(1)}-i \omega_{2}^{E} \phi_{(2)}}\left[1+\frac{4 \pi^{2}}{\left(\phi_{(1)}-\phi_{(2)}\right)^{2}-4 \pi^{2}}\right] .
\end{aligned}
$$

Note that even though we have a sum of two diagrams, we do not have a factor of 2 . This is due to the fact that the two D-instantons are identical and therefore exchanging them does not give a new contribution as long as we integrate $\phi_{(1)}$ and $\phi_{(2)}$ over the entire 
real line. Since we are working in the Euclidean theory, there are no factors of $i$ in the overall normalization. On the right hand side of (7.2), the contribution of the first term inside the square bracket has separate factors of $\delta\left(\omega_{1}^{E}\right)$ and $\delta\left(\omega_{2}^{E}\right)$, and corresponds to a disconnected diagram in the closed string effective field theory with a pair of D-instanton type interaction vertices. We shall not consider this contribution any further.

Figure 8(b) represents an amplitude where the incoming closed string vertex operator is inserted at the center of the disk whose boundary lies on instanton 1 and the outgoing closed string vertex operator is inserted at the center of the disk whose boundary also lies on instanton 1. Figure 8(c) represents a similar contribution where the boundaries of both disks lie on instanton 2 . This is related to figure 8 (b) by the exchange of the positions of the two D-instantons and give the same result after integration over the positions. Therefore we shall combine the two contributions. The result takes the form:

$$
\begin{aligned}
& \mathbf{N}^{2} e^{-2 / g_{s}} \int d \phi_{(1)} d \phi_{(2)} e^{-i \omega_{1}^{E} \phi_{(1)}-i \omega_{2}^{E} \phi_{(1)}} 2 \sinh \left(2 \pi P_{1}\right) 2 \sinh \left(2 \pi P_{2}\right)\left\{\exp \left[\mathcal{A}_{12}\right]-1\right\} \\
& \quad=\mathbf{N}^{2} e^{-2 / g_{s}} 4 \sinh \left(2 \pi P_{1}\right) \sinh \left(2 \pi P_{2}\right) \int d \phi_{(1)} d \phi_{(2)} e^{-i \omega_{1}^{E} \phi_{(1)}-i \omega_{2}^{E} \phi_{(1)}} \frac{4 \pi^{2}}{\left(\phi_{(1)}-\phi_{(2)}\right)^{2}-4 \pi^{2}} .
\end{aligned}
$$

Again we see that despite having two contributions there is no factor of two, since exchanging two identical D-instantons does not give a new contribution as long as we integrate $\phi_{(1)}$ and $\phi_{(2)}$ individually over the entire range.

Introducing new variables $\phi$ and $\chi$ via,

$$
\phi_{(1)}=\phi+\frac{1}{2} \chi, \quad \phi_{(2)}=\phi-\frac{1}{2} \chi,
$$

and using the Lorentzian prescription (5.15) for dealing with the singularities at $\chi=2 \pi$, we can express the sum of (7.2) and (7.3), leaving out the first term inside the square bracket of (7.2), as

$$
\begin{aligned}
& \mathbf{N}^{2} e^{-2 / g_{s}} 4 \sinh \left(2 \pi P_{1}\right) \sinh \left(2 \pi P_{2}\right) \int d \phi d \chi e^{-i \phi\left(\omega_{1}^{E}+\omega_{2}^{E}\right)} \frac{4 \pi^{2}}{\chi^{2}-4 \pi^{2}+i \epsilon} \\
& \times\left[e^{-i \chi\left(\omega_{1}^{E}-\omega_{2}^{E}\right) / 2}+e^{-i \chi\left(\omega_{1}^{E}+\omega_{2}^{E}\right) / 2}\right] \\
& =\mathbf{N}^{2} e^{-2 / g_{s}} 16 \pi^{2} \sinh \left(2 \pi P_{1}\right) \sinh \left(2 \pi P_{2}\right) 2 \pi \delta\left(\omega_{1}^{E}+\omega_{2}^{E}\right) \int \frac{d \chi}{\chi^{2}-4 \pi^{2}+i \epsilon}\left[e^{-i \chi\left(\omega_{1}^{E}-\omega_{2}^{E}\right) / 2}+1\right] .
\end{aligned}
$$

We shall now analytically continue $\omega_{i}^{E}$ to the Lorentzian energies $\omega_{i}=i \omega_{i}^{E}$ by replacing $\omega_{i}^{E}$ by $e^{-i \theta} \omega_{i}$ with real $\omega_{i}$ and deforming $\theta$ from 0 to $\pi / 2$. This can be done by simultaneously deforming the $\chi$ integration contour as $\chi=e^{i \theta} u$ with $u$ real, so that $e^{ \pm i \omega_{i}^{E} \chi}$ remain bounded along the integration contour and the integral converges for all $\theta$. At $\theta=\pi / 2$ we have,

$$
\omega_{i}^{E}=-i \omega_{i}, \quad \chi=i u .
$$

Now as discussed in section 3.1 under this deformation the amplitudes in the Euclidean theory directly get mapped to the T-matrix elements without any extra factor of $i$. Therefore 


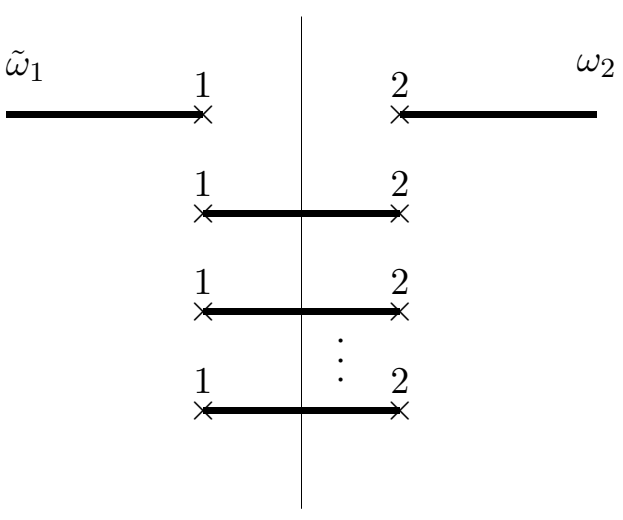

(a)

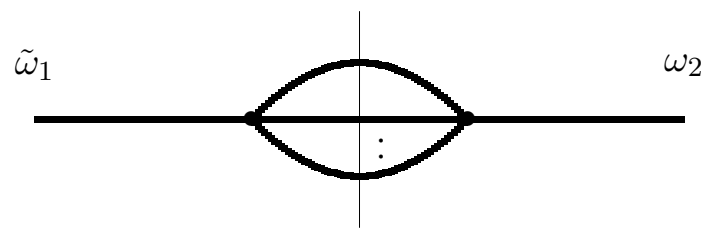

(b)

Figure 9. (a) A cut diagram in open closed string field theory that can contribute to the imaginary part of the leading two instanton contribution to $1 \rightarrow 1$ closed string amplitude. The thin vertical line represents the cut. (b) The same cut diagram represented in the closed string effective field theory, with the $\bullet$ 's representing the interaction vertices of this effective field theory.

we have:

$$
\begin{aligned}
T_{2-\text { inst }}= & i \mathbf{N}^{2} e^{-2 / g_{s}} 16 \pi^{2} \sinh \left(\pi\left|\omega_{1}\right|\right) \sinh \left(\pi\left|\omega_{2}\right|\right) 2 \pi \delta\left(\omega_{1}+\omega_{2}\right) \\
& \int d u \frac{1}{-u^{2}-4 \pi^{2}}\left[e^{i \omega_{2} u}+1\right] \\
= & -i \mathbf{N}^{2} e^{-2 / g_{s}} 8 \pi^{2} \sinh ^{2}\left(\pi \omega_{2}\right) 2 \pi \delta\left(\omega_{1}+\omega_{2}\right)\left(1+e^{-2 \pi\left|\omega_{2}\right|}\right),
\end{aligned}
$$

where we have used the relation $\omega_{1}=-\omega_{2}$ and the on-shell condition $\left|\omega_{i}\right|=2 P_{i}$ to simplify the coefficient of $\delta\left(\omega_{1}+\omega_{2}\right)$. As will be discussed in section 7.4 , this agrees with the result of [12] after appropriate change of notation.

Before we proceed, we make a few remarks on the result given in (7.7):

1. We can also obtain (7.7) by first evaluating the $\chi$ integration in (7.5) and then doing analytic continuation, keeping in mind that the analytic continuation needs to be done via the second and the fourth quadrants of the $\omega_{i}^{E}$ plane, or equivalently, the first and the third quadrants of the $\omega_{i}$ plane. Therefore positive (negative) $\omega_{i}^{E}$ analytically continues to positive (negative) $\omega_{i}$.

2. Appearance of $\left|\omega_{2}\right|$ in (7.7) shows that the amplitude is non-analytic at $\omega_{2}=0$. This singularity can be traced to possible divergences from the large $|\chi|$ region in (7.5) although the integral is finite, sufficient number of derivatives with respect to $\omega_{2}^{E}$ will generate powers of $\chi$ in the numerator and make the integral diverge at $\omega_{i}^{E}=0$. If on the left hand sides of (7.2) and (7.3) we had performed the $\chi$ integration before doing the $t$ integration in the expression for $\mathcal{A}_{12}$ given in (7.1), then the divergence would come from the $t \simeq 0$ region, associated with closed string degeneration. In string field theory this non-analyticity can be traced to the singularities associated with the cut diagrams shown in figure 9. 
3. In contrast, we shall see in section 7.2 that the contribution to the closed string effective action, given in (7.16), is an analytic function of $\omega_{2}$ in the complex $\omega_{2}$ plane since the integrand in the $\chi$ integration will have exponential suppression from the large $|\chi|$ region. This can be traced to the fact that in the construction of the effective action we shall use $A_{12}$ defined in (5.1) where the $t$ integral has a lower cut-off.

4. From (7.7) we see that even if $\mathbf{N}$ is taken to be real, as is needed for unitarity of the one instanton amplitude, the 2-instanton amplitude acquires an imaginary part. This by itself does not signal violation of unitarity — we need to check if the imaginary part arises from the cutting rules. We shall see in section 7.2 that part of (7.7) does come from sum over cuts of the Feynman diagrams of string field theory shown in figure 9 . The rest can be traced to the $i \epsilon$ factor in (7.5) that produces an imaginary part of the two instanton interaction vertex in closed string effective field theory. This is turn will show that the amplitude violates unitarity.

Let us now compute the same amplitude using the unitary prescription. This is done by using the identify (5.16) to express the amplitude as a sum of (7.5) and an additional contribution proportional to $\delta\left(\chi^{2}-4 \pi^{2}\right)$ in the integrand. For these extra terms, we can evaluate the $\chi$ integral using this $\delta$-function and then replace $\omega_{i}^{E}$ by $-i \omega_{i}$ as given in (7.6). This gives, for $\omega_{2}>0$ :

$$
i \mathbf{N}^{2} e^{-2 / g_{s}} 4 \pi^{2} \sinh ^{2}\left(\pi \omega_{2}\right) 2 \pi \delta\left(\omega_{1}+\omega_{2}\right)\left(2+e^{-2 \pi \omega_{2}}+e^{2 \pi \omega_{2}}\right) .
$$

Adding this to (7.7) we get the amplitude in the unitary prescription:

$$
T_{2-\mathrm{inst}}^{\prime}=i \mathbf{N}^{2} e^{-2 / g_{s}} 4 \pi^{2} \sinh ^{2}\left(\pi \omega_{2}\right) 2 \pi \delta\left(\omega_{1}+\omega_{2}\right)\left(e^{2 \pi\left|\omega_{2}\right|}-e^{-2 \pi\left|\omega_{2}\right|}\right) .
$$

We shall check in section 7.3 that this imaginary contribution can be traced fully to the cut diagrams of figure 9 , and is therefore consistent with unitarity.

We shall now discuss an alternate procedure for arriving at (7.9) that will be easy to generalize to multi-instanton amplitudes. For this, note that under complex conjugation, an expression like (7.5) will have the sign of its $i \epsilon$ reversed. The sign of the exponent also changes, but this can be undone by a $\chi \rightarrow-\chi$ change of integration variable. Therefore to get the result for the unitary prescription, we can take the result for the Lorentzian prescription, analytically continue it back to the Euclidean external momenta, complex conjugate the result, continue the result back to the Lorentzian momenta, and finally average over the initial and the final results. This can be achieved directly in the Lorentzian prescription by complex conjugation and change of the signs of the exponents ${ }^{11}$ in the terms involving exponentials of the energies and then averaging over the initial and the

\footnotetext{
${ }^{11}$ Note that this is a formal substitution and does not entail evaluating the result for opposite sign of the energy. For example, although in (7.7) the final factor inside the parentheses is $e^{-2 \pi\left|\omega_{2}\right|}$ and therefore vanishes under antisymmetrization under $\omega_{2} \rightarrow-\omega_{2}$, this is not relevant for our discussion. This is because the euclidean continuation of $e^{-2 \pi\left|\omega_{2}\right|}$ is $e^{-2 \pi i\left|\omega_{2}^{E}\right|}$, and the exponent changes sign under complex conjugation. Therefore when we analytically continue it back to the Lorentzian signature, we get $e^{2 \pi\left|\omega_{2}\right|}$ and the sign of the exponent still changes.
} 
Figure 10. The closed string contribution to the annulus partition function with different boundary conditions on the two boundaries.

final results. For example, since complex conjugation of (7.7) produces an overall sign due to the presence of the factor of $i$, this procedure simply anti-symmetrizes the final factor inside the parenthesis under the replacement $\left|\omega_{2}\right| \rightarrow-\left|\omega_{2}\right|$, producing the factor $\left(e^{-2 \pi\left|\omega_{2}\right|}-e^{2 \pi\left|\omega_{2}\right|}\right) / 2$. This reproduces (7.9).

\subsection{Feynman diagram representation of the amplitude}

We shall now describe how the results of section 7.1 can be organized into sum of Feynman diagrams in the open closed string field theory. For this we need to first split the annulus contribution $\mathcal{A}_{12}$ given in (7.1) into the contribution from closed string exchange diagram shown in figure 10, where a pair of disk one point functions are connected by the closed string propagator, and the contribution $A_{12}$ from the open string loops. Contribution from figure 10 is given by:

$$
\begin{aligned}
& \int_{-\infty}^{\infty} \frac{d \omega_{E}}{2 \pi} \int_{0}^{\infty} d P e^{-i \omega_{E}\left(\phi_{(1)}-\phi_{(2)}\right)} \frac{1}{P^{2}+\frac{\omega_{E}^{2}}{4}} e^{-2 b\left(P^{2}+\omega_{E}^{2} / 4\right)} 4 \sinh ^{2}(2 \pi P) \\
& \quad=\int_{2 b}^{\infty} d s \int_{-\infty}^{\infty} \frac{d \omega_{E}}{2 \pi} \int_{0}^{\infty} d P e^{-i \omega_{E}\left(\phi_{(1)}-\phi_{(2)}\right)} e^{-s\left(P^{2}+\omega_{E}^{2} / 4\right)}\left(e^{4 \pi P}+e^{-4 \pi P}-2\right)
\end{aligned}
$$

where in the first step we have used the off-shell closed string one point function on the disk as given in (6.3) and the closed string propagator given on the left hand side of (6.12) after euclidean rotation and in the second step we have used the Schwinger parameter representation of the propagator. Since the integrand is even function of $P$, we can extend the range of integration over $P$ from $-\infty$ to $\infty$ at the cost of including a factor of $1 / 2$, and then carry out integration over $\omega_{E}$ and $P$ by standard rules of Gaussian integration. After changing variable to

$$
t \equiv 2 \pi / s
$$

the final result may be written as,

$$
\int_{0}^{a} d t t^{-1}\left\{e^{-t\left(\chi^{2}-4 \pi^{2}\right) / 2 \pi}-e^{-t \chi^{2} / 2 \pi}\right\}, \quad \chi \equiv \phi_{(1)}-\phi_{(2)},
$$

where,

$$
a \equiv \frac{\pi}{b} .
$$

Comparing this with (7.1) we see that the remaining contribution, that was called $A_{12}$ in (5.1) and that enters the definition of the two instanton interaction vertex, is given by:

$$
A_{12}=\int_{a}^{\infty} d t t^{-1}\left\{e^{-t\left(\chi^{2}-4 \pi^{2}\right) / 2 \pi}-e^{-t \chi^{2} / 2 \pi}\right\} .
$$

Note that $A_{12}$ differs from $\mathcal{A}_{12}$ given in (7.1) in that in (7.14) the lower limit on the $t$ integral is $a$ while in (7.1) the lower limit is 0 . We shall simplify the analysis by working in 


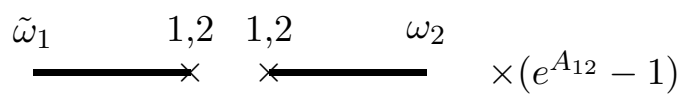

(a)

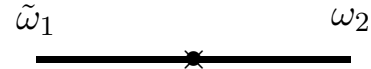

(b)

Figure 11. (a) A Feynman diagram in the open closed string field theory giving two instanton contribution to the closed string two point function. 1,2 label at the vertex means that it can refer to either the first or the second D-instanton. (b) Representation of the same Feynman diagram in closed string effective field theory. represents a two instanton interaction vertex in the closed string effective field theory.

the small $a$ limit — but this will still be different from the $a=0$ case. In this case for any finite $\chi \equiv \phi_{(1)}-\phi_{(2)}$ the difference between $A_{12}$ and $\mathcal{A}_{12}$ becomes small for small $a$, but for any small but fixed $a$, the contribution to (7.14) for large $\chi$ gets suppressed as $e^{-a \chi^{2} / 2 \pi}$, while there is no such suppression for $\mathcal{A}_{12}$.

With this result, we are now ready to compute the contribution to the closed string two point function from various Feynman diagrams of open closed string field theory. We first consider the Feynman diagram of figure 11. Figure 11(a) shows the Feynman diagram representing this contribution in open closed string field theory, while figure 11(b) shows the same contribution as a Feynman diagram in closed string effective field theory. This contribution is related to the full result for the same amplitude to this order, given by (7.5), by a multiplication function $f(\chi)$ in the integrand, where $f(\chi)$ is some even, real function of $\chi$ that takes value 1 for $a \chi^{2} \ll 1$, but has a suppression factor of $e^{-a \chi^{2} / 2 \pi}$ for $a \chi^{2} \gg 1$. Therefore this may be written as:

$$
\mathbf{N}^{2} e^{-2 / g_{s}} 16 \pi^{2} \sinh \left(2 \pi P_{1}\right) \sinh \left(2 \pi P_{2}\right) 2 \pi \delta\left(\omega_{1}^{E}+\omega_{2}^{E}\right) \int \frac{d \chi}{\chi^{2}-4 \pi^{2}+i \epsilon} f(\chi)\left[e^{-i \chi\left(\omega_{1}^{E}-\omega_{2}^{E}\right) / 2}+1\right] .
$$

Due to the exponential suppression of the integrand for large $\chi$, we can keep the $\chi$ integration contour along the real axis even when $\omega_{i}^{E}$ are complex. Finiteness of the integral (and its derivatives with respect to $\omega_{i}^{E}$ ) shows that the integral is an analytic function of $\omega_{i}^{E}$ in the whole complex plane. Therefore its value for $\omega_{i}^{E}=-i \omega_{i}$ for real $\omega_{i}$ can be obtained simply by substitution of $\omega_{i}^{E}$ by $-i \omega_{i}$ :

$$
\mathbf{N}^{2} e^{-2 / g_{s}} 16 \pi^{2} \sinh \left(2 \pi P_{1}\right) \sinh \left(2 \pi P_{2}\right) 2 \pi \delta\left(\omega_{1}+\omega_{2}\right) \int \frac{d \chi}{\chi^{2}-4 \pi^{2}+i \epsilon} f(\chi)\left[e^{-\chi\left(\omega_{1}-\omega_{2}\right) / 2}+1\right] \text {. }
$$

Here we have used the result of section 3.1 that in relating the Euclidean action to the Lorentzian action, or the Euclidean Green's function to the T-matrix elements, the momentum conserving $\delta$-functions have their arguments replaced by Lorentzian momenta, and there are no extra factors of $i$ in the overall normalization. The reality of the amplitude requires it to be invariant under the simultaneous operation of complex conjugation and change of sign of the $\omega_{i}$ 's. Since the latter operation can be undone by a $\chi \rightarrow-\chi$ change of variable in the integration, the only source of the imaginary part of this amplitude is the $i \epsilon$ in the denominator. This gives a factor proportional to $\delta\left(\chi^{2}-4 \pi^{2}\right)$. Since in the small $a$ limit, $f(\chi)=1$ for finite $\chi$, we can replace $f(\chi)$ by 1 at $\chi= \pm 2 \pi$. This gives the 


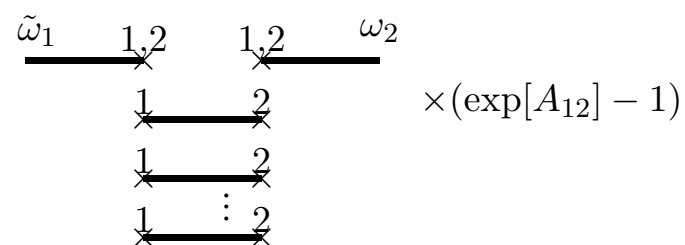

(a)

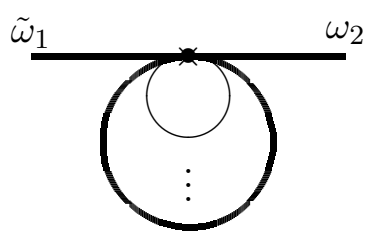

(b)

Figure 12. (a) A set of Feynman diagrams in open closed string field theory that can be part of the leading two instanton contribution to $1 \rightarrow 1$ closed string amplitude. The symbol 1,2 at the $\times$ denotes that the vertex may describe either type 1 D-instanton or type 2 D-instanton. (b) The same diagram represented in the closed string effective field theory, with representing a two instanton interaction vertex in the closed string effective field theory. As argued in the text, the contribution to these diagrams is suppressed in the small $a$ limit.

imaginary part of the amplitude to be:

$$
-i \mathbf{N}^{2} e^{-2 / g_{s}} 4 \pi^{2} \sinh ^{2}\left(\pi \omega_{2}\right) 2 \pi \delta\left(\omega_{1}+\omega_{2}\right)\left[e^{2 \pi \omega_{2}}+e^{-2 \pi \omega_{2}}+2\right],
$$

where we have used the on-shell condition $P_{i}=\left|\omega_{i}\right| / 2$.

We now make a few comments on the result (7.17).

1. (7.17) differs from (7.7). This difference can be traced to the difference between $\mathcal{A}_{12}$ and $A_{12}$ provided by the damping factor $f(\chi)$. The reason that the argument given above cannot be applied to the full amplitude (7.5) is that (7.16) does not make sense without the damping factor $f(\chi)$ since the integral diverges for $\chi \rightarrow \infty$ or $\chi \rightarrow-\infty$ depending in the sign of $\omega_{1}-\omega_{2}$. When we regulate this divergence we may get additional imaginary terms. Indeed, these divergences are related to on-shell closed string propagation in the intermediate state shown in figure 14, and the imaginary part associated with this will be computed in (7.21).

2. Since figure $11(\mathrm{~b})$ represents a 2-instanton interaction vertex of the closed string effective field theory with two external closed strings, the $i$ in (7.17) shows that the 2instanton action of the closed string effective field theory has imaginary contribution. This will lead to violation of unitarity. We could explicitly write down the term in the effective action corresponding to (7.17) following (6.2), but will not do so.

3. As already discussed, the expression (7.16) is analytic in the full complex $\omega_{2}$ plane, since due to the exponential suppression factor proportional to $e^{-a \chi^{2} / 2 \pi}$ hidden inside $f(\chi)$, the integration over $\chi$ converges for any complex $\omega_{2}$. This is what is expected of any term in the closed string effective action. This is to be contrasted with the amplitude given in (7.7) that has non-analyticity at $\omega_{2}=0$ due to the cuts associated with figure 9 .

Let us now examine other Feynman diagrams that can contribute to this amplitude. A second class of Feynman diagrams that can contribute at the same order has been shown in figure 12. The contribution from these diagrams will be given by an integral of the 


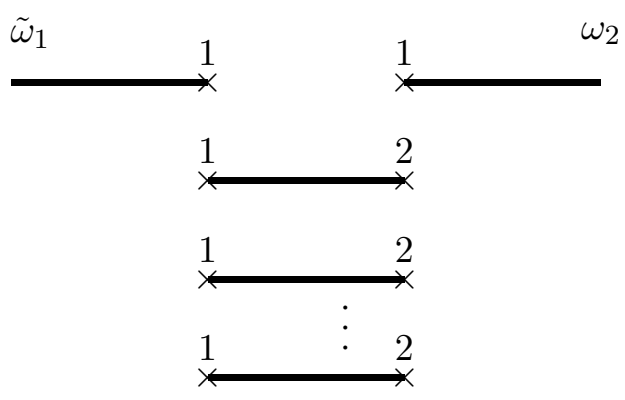

(a)

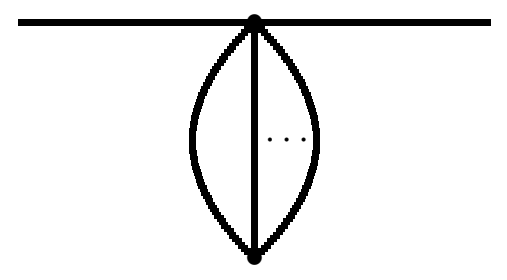

(b)

Figure 13. (a) Examples of Feynman diagrams of open closed string field theory giving part of the leading 2-instanton contribution to the closed string 2-point amplitude. We also have a similar diagram where the pair of 1's in the top line are replaced by a pair of 2's. (b) Representation of the same diagram in the closed string effective field theory.

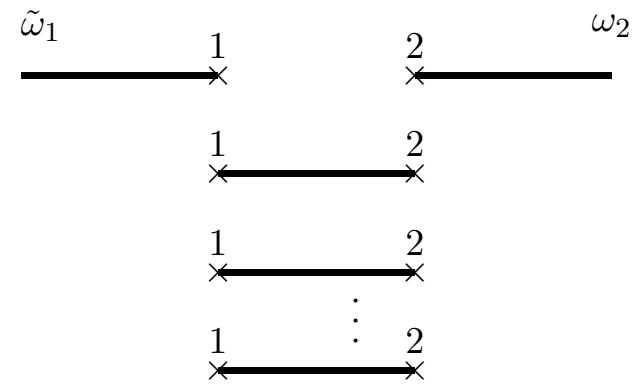

(a)

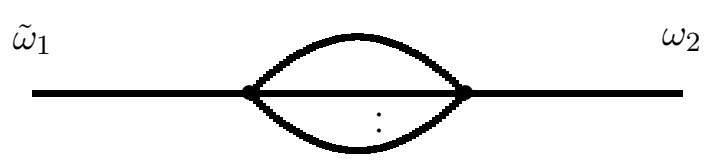

(b)

Figure 14. (a) A set of Feynman diagrams in open closed string field theory that can be part of the leading 2-instanton contribution to the closed string 2-point function. (b) The same Feynman diagram represented in the closed string effective field theory.

form (7.16), but the integrand will be multiplied by one factor of (7.12) for every extra closed string propagator. Since (7.12) is a real and even function of $\chi$, the same argument as for (7.16) tells us that the imaginary part will come from the points $\chi= \pm 2 \pi$. Now as we take $a \rightarrow 0, b \rightarrow \infty$ limit, the term inside the square bracket in (7.16) remains bounded, while (7.12) vanishes in this limit. Therefore we conclude that the amplitudes shown in figure 12 do not have any imaginary part in the large $b$ limit. This is consistent with the cutting rules since figure 12(b) does not admit any cut. This however was not guaranteed from the start since we have not established the reality of the two instanton interaction vertex that appears in figure 12(b).

Figure 11 and figure 12 take into account all the Feynman diagrams that have one or more factor of $A_{12}$. Now we turn to the Feynman diagrams without this factor. We shall divide these into two classes of Feynman diagrams, shown in figure 13 and figure 14. From the representation of these diagrams in the closed string effective field theory, we see that they are constructed from two one instanton interaction vertices in the closed string 
effective field theory. Since we have taken $\mathbf{N}$ to be real, the one instanton interaction vertices do not violate unitarity, and therefore the imaginary parts of these diagrams will be given by the sum over all cut diagrams. It is easy to see from figure 13(b) that these diagrams do not admit any cut and therefore do not have any imaginary part. The cut diagram of figure 14 has been shown in figure 9. Its contribution, computed using the usual cutting rules, is given by:

$$
\begin{aligned}
& i \mathbf{N}^{2} e^{-2 / g_{s}} 2 \pi \delta\left(\omega_{1}+\omega_{2}\right) 4 \sinh \left(\pi\left|\omega_{1}\right|\right) \sinh \left(\pi\left|\omega_{2}\right|\right) \sum_{n=1}^{\infty} \frac{1}{n !} \int_{P_{i}>0} d P_{1} \cdots d P_{n} \\
& \int_{e_{i}>0} \frac{d e_{1}}{2 \pi} \cdots \frac{d e_{n}}{2 \pi} 2 \pi \delta\left(e_{1}+\cdots e_{n}-\left|\omega_{2}\right|\right) \prod_{i=1}^{n}\left\{2 \pi \delta\left(\frac{e_{i}^{2}}{4}-P_{i}^{2}\right) 4 \sinh ^{2}\left(2 \pi P_{i}\right)\right\} .
\end{aligned}
$$

Note that we have written down the formula in a way that is valid both for positive and negative $\omega_{2}$. We can simplify this by first carrying out the integration over the $P_{i}$ 's using the delta functions, and then use the identity,

$$
\sum_{n=1}^{\infty} \frac{4^{n}}{n !} \int_{e_{i}>0} \frac{d e_{1}}{e_{1}} \cdots \frac{d e_{n}}{e_{n}} 2 \pi \delta\left(e_{1}+\cdots e_{n}-\left|\omega_{2}\right|\right) \prod_{i=1}^{n} \sinh ^{2}\left(\pi e_{i}\right)=4 \pi^{2} \sinh \left(2 \pi\left|\omega_{2}\right|\right) .
$$

(7.19) can be proved by multiplying both sides by $e^{-\mu\left|\omega_{2}\right|}$, integrating over $\omega_{2}$ from 0 to $\infty$ (or from $-\infty$ to 0 ) and showing that the two sides agree for arbitrary $\mu>2 \pi$. This reduces (7.18) to,

$$
i \mathbf{N}^{2} e^{-2 / g_{s}} 2 \pi \delta\left(\omega_{1}+\omega_{2}\right) 4 \sinh ^{2}\left(\pi \omega_{2}\right) 4 \pi^{2} \sinh \left(2 \pi\left|\omega_{2}\right|\right) .
$$

(7.20) gives twice the contribution to the imaginary part of the T-matrix. Therefore the contribution from this cut diagram to the imaginary part of the T-matrix is given by:

$$
i \mathbf{N}^{2} e^{-2 / g_{s}} 8 \pi^{2} 2 \pi \delta\left(\omega_{1}+\omega_{2}\right) \sinh ^{2}\left(\pi \omega_{2}\right) \sinh \left(2 \pi\left|\omega_{2}\right|\right) .
$$

Adding (7.17) and (7.21) we get the total contribution to the imaginary part of the closed string 2-point amplitude to this order:

$$
-i \mathbf{N}^{2} e^{-2 / g_{s}} 8 \pi^{2} \sinh ^{2}\left(\pi \omega_{2}\right) 2 \pi \delta\left(\omega_{1}+\omega_{2}\right)\left(1+e^{-2 \pi\left|\omega_{2}\right|}\right) .
$$

This agrees with (7.7). Note however that if we were using the ordinary cutting rules of a field theory with real effective action, then we shall only have the contribution from the cut diagram of figure 9 . Its contribution to the imaginary part of the T-matrix, given by (7.21), differs from the full answer (7.7). Therefore the usual cutting rules fail. The difference can be accounted for by the imaginary part of figure 11 given in (7.17), which in turn, originates from the violation of reality condition by the 2 -instanton interaction vertex of the closed string effective field theory. This signals the violation of unitarity within the closed string sector.

In conclusion we see that even if we had taken $\mathbf{N}$ to be real, unitarity is violated. This can be traced to the appearance of the open string tachyons below a critical separation 
of the two D-instantons, and our use of Lorentzian prescription for dealing with the associated singularity. We also note that even though the contribution from the individual Feynman diagrams depends on the string field theory parameter $a$ (or equivalently $b$ ), the contribution from the cut diagram is given by the product of physical amplitudes in the single instanton sector, and is independent of the string field theory parameter. On the other hand the imaginary part of the original 2-point amplitude, given in (7.7), is also independent of the string field theory parameters. Therefore the violation of cutting rules is a physical effect that cannot be changed by changing the string field theory parameters.

\subsection{Unitarity restoration via unitary prescription}

We shall now examine what happens if we use the unitary prescription for dealing with the singularities associated with the critical separation of the D-instantons where a tachyon appears. The leading 2-instanton contribution to the 2-point function of closed string tachyons, computed with this prescription, is given in (7.9). It is easy to see that this agrees with the prediction of the cutting rules, given in (7.21), for the imaginary part of the amplitude.

This can be also seen at the level of the effective action. It follows from the analysis in section 7.2 that the only source of imaginary term in the effective action is in the part of the amplitude given in (7.17), which will be absent if we used the unitary prescription instead of the Lorentzian prescription in (7.16). Therefore the resulting effective action will be real.

\subsection{Connection to the matrix model}

In this section we shall compare our results to that in [12]. During the analysis of $k$ instanton amplitudes for $k \geq 2$, [12] used the prescription of integrating over the zero modes directly in the Lorentzian space without any factor of $i$. We shall first show why this is consistent with our procedure of defining the integral in the Euclidean space and picking up appropriate factors of $i$ if we need to rotate the integral, as was done while arriving at (7.7). For this we note that in our convention, for the general case of $k$ instantons we shall have an overall normalization factor $\mathbf{N}^{k}=(-i \mathcal{N})^{k}$, where $\mathcal{N}=i \mathbf{N}=-1 /\left(8 \pi^{2}\right)$ in the notation of [12]. On the other hand while computing the amplitude we have to integrate over $(k-1)$ euclidean variables labelling the differences in the Euclidean time coordinates of the D-instantons - integration over the variable labelling the average time coordinate produces the energy conserving delta function and has already been discussed in section 3.1. After rotation to Lorentzian variables, this gives a factor of $i^{k-1}$. To get the S-matrix from the T-matrix we have an extra factor of $i$. Therefore the net normalization, in the convention where we integrate the zero modes along the Lorentzian direction, is given by:

$$
(-i \mathcal{N})^{k} i^{k-1} i=\mathcal{N}^{k}
$$

This agrees with the prescription of [12] of integrating over the zero modes in Lorentzian space and using normalization factor $\mathcal{N}^{k}$ for $k$ instanton amplitude. 
We can test this by comparing the results for the leading 2-instanton contribution to the two point function of closed string tachyons. We use the relation $\mathbf{N}=-i \mathcal{N}$ to rewrite (7.7) as:

$$
S_{2-\text { inst }}=i T_{2-\text { inst }}=-\mathcal{N}^{2} e^{-2 / g_{s}} 8 \pi^{2} \sinh ^{2}\left(\pi \omega_{2}\right) 2 \pi \delta\left(\omega_{1}+\omega_{2}\right)\left(1+e^{-2 \pi\left|\omega_{2}\right|}\right) .
$$

This agrees with the result of [12] for the leading two instanton contribution to the closed string 2-point function, and agrees with the matrix model results after adding the contribution from the $(2,1)$ D-instanton.

This is the result using Lorentzian prescription for the integration contour of the zero modes. As we have argued in section 5.5, this prescription leads to non-unitary amplitudes even when we expect the theory to be unitary, e.g. for D-instanton anti-Dinstanton contribution to the amplitudes in type IIB string theory. The unitary prescription gives unitary amplitudes in these theories. Even though in two dimensional bosonic string theory we do not expect a unitary amplitude, and indeed unitarity is already violated by the one instanton amplitudes by having an imaginary $\mathbf{N}$, we shall now explore what results we get if we use the unitary prescription in two dimensional string theory. We have already seen that for the two instanton contribution to the two point amplitude, we shall have a missing term (7.17) in the unitary prescription. Multiplying this by $i$ to translate this to a contribution to the S-matrix, using $\mathbf{N}=-i \mathcal{N}$, and using the on-shell condition $P_{i}=\left|\omega_{i}\right| / 2$ for $i=1,2$, we get the missing term to be,

$$
\begin{gathered}
-\mathcal{N}^{2} e^{-2 / g_{s}} 4 \pi^{2} \sinh ^{2}\left(\pi \omega_{2}\right) 2 \pi \delta\left(\omega_{1}+\omega_{2}\right)\left[e^{2 \pi \omega_{2}}+e^{-2 \pi \omega_{2}}+2\right] \\
=-\frac{1}{16 \pi^{2}} e^{-2 / g_{s}} 2 \pi \delta\left(\omega_{1}+\omega_{2}\right) \sinh ^{2}\left(2 \pi \omega_{2}\right)
\end{gathered}
$$

where we have used $\mathcal{N}=-1 /\left(8 \pi^{2}\right)$.

Since [12] got agreement with the matrix model results, if we want the unitary prescription to also agree with the matrix model results, we must have some other source of the contribution (7.25) to compensate for this missing term. To this end we note that [12] parametrized the contribution to the same S-matrix element from $(2,1)$ ZZ-instanton as:

$$
4 \mathcal{N}_{2} e^{-2 / g_{s}} 2 \pi \delta\left(\omega_{1}+\omega_{2}\right) \sinh ^{2}\left(2 \pi \omega_{2}\right),
$$

for some constant $\mathcal{N}_{2}$. Comparing (7.25) and (7.26) we see that the missing contribution (7.25) may be compensated by a shift in $\mathcal{N}_{2}$ of the form $\mathcal{N}_{2} \rightarrow \mathcal{N}_{2}-1 /\left(64 \pi^{2}\right)$. Now the result of [12] for $\mathcal{N}_{2}$ was $3 /\left(64 \pi^{2}\right)$. Therefore our new result for $\mathcal{N}_{2}$ with the unitary prescription is:

$$
\mathcal{N}_{2}=\frac{1}{32 \pi^{2}} .
$$

We shall now verify that the same shift in $\mathcal{N}_{2}$ also correctly reproduces the 2 -instanton contribution to the $n$-point amplitude. The difference between the Lorentzian and unitary prescription for the $n$-point amplitude comes from different treatment of the analog of the Feynman diagram shown in figure 11(a), except that instead of having just two external closed strings, we now have $n$ external closed strings, each of which can connect to a type 
1 D-instanton vertex or a type 2 D-instanton vertex. In the Lorentzian prescription the result of this diagram can be written down in a manner analogous to (7.16):

$$
\frac{1}{2} \mathbf{N}^{2} e^{-2 / g_{s}} 4 \pi^{2}\left\{\prod_{i=1}^{n} 2 \sinh \left(2 \pi P_{i}\right)\right\} 2 \pi \delta\left(\sum_{i=1}^{n} \omega_{i}\right) \int \frac{d \chi}{\chi^{2}-4 \pi^{2}+i \epsilon} f(\chi) \sum_{S \subset\{1, \cdots n\}} e^{-\chi \sum_{i \in S} \omega_{i}} .
$$

Here $S$ denotes a subset of $\{1, \cdots, n\}$ labelling the collection of external closed strings that connect to type $1 \mathrm{D}$-instanton vertices, the rest of the external closed strings connect to type 2 D-instanton vertices. The sum over $S$ can be easily performed yielding $\prod_{i}\left\{2 \cosh \left(\chi \omega_{i} / 2\right)\right\}$. Using on-shell condition $P_{i}=\left|\omega_{i}\right| / 2$, we can rewrite (7.28) as:

$$
\frac{1}{2} \mathbf{N}^{2} e^{-2 / g_{s}} 4 \pi^{2}\left\{\prod_{i=1}^{n} 2 \sinh \left(\pi\left|\omega_{i}\right|\right)\right\} 2 \pi \delta\left(\sum_{i=1}^{n} \omega_{i}\right) \int \frac{d \chi}{\chi^{2}-4 \pi^{2}+i \epsilon} f(\chi) \prod_{i}\left\{2 \cosh \left(\chi \omega_{i} / 2\right)\right\} .
$$

In the unitary prescription, instead of using $i \epsilon$ we use the average of $i \epsilon$ and $-i \epsilon$ prescription to carry out the $\chi$ integration. The difference is proportional to $\delta\left(\chi^{2}-4 \pi^{2}\right)$. Using the result $f( \pm 2 \pi)=1$, we can write the extra contribution in the Lorentzian prescription as,

$$
-i \mathbf{N}^{2} e^{-2 / g_{s}} \pi^{2}\left\{\prod_{i=1}^{n} 2 \sinh \left(2 \pi\left|\omega_{i}\right|\right)\right\} 2 \pi \delta\left(\sum_{i=1}^{n} \omega_{i}\right) .
$$

Multiplying this by $i$ and using $\mathbf{N}=-i \mathcal{N}=i /\left(8 \pi^{2}\right)$, we get the extra contribution to the S-matrix in the Lorentzian prescription:

$$
-\frac{1}{64 \pi^{2}} e^{-2 / g_{s}}\left\{\prod_{i=1}^{n} 2 \sinh \left(2 \pi\left|\omega_{i}\right|\right)\right\} 2 \pi \delta\left(\sum_{i=1}^{n} \omega_{i}\right) .
$$

On the other hand, the contribution to the $n$-instanton amplitude due to the $(2,1)$ ZZinstanton was computed in [12] to be:

$$
\mathcal{N}_{2} e^{-2 / g_{s}}\left\{\prod_{i=1}^{n} 2 \sinh \left(2 \pi\left|\omega_{i}\right|\right)\right\} 2 \pi \delta\left(\sum_{i=1}^{n} \omega_{i}\right) .
$$

Therefore the missing contribution (7.31) in the unitary prescription can be compensated by a shift $\mathcal{N}_{2} \rightarrow \mathcal{N}_{2}-1 /\left(64 \pi^{2}\right)$. This again leads us back to (7.27) as the new value of $\mathcal{N}_{2}$ in the unitary prescription.

\subsection{Three instanton amplitude in the unitary prescription}

We shall now study the effect of using unitary prescription for the three instanton amplitude. For this we shall use the expressions for various amplitudes computed in [12] in the Lorentzian prescription and apply the procedure described at the end of section 7.1 to get the result in the unitary prescription. In order to avoid the proliferation of the absolute value symbol, we shall write all subsequent formulæ in this subsection for an incoming closed string of energy $\tilde{\omega}_{1}=-\omega_{1}>0$ and an outgoing closed string of energy $\omega_{2}>0$.

As already emphasized, there are two differences between the notation in [12] and the ones used in this paper. First of all [12] gave the results for the S-matrix elements while 
the various manipulations we have described is for the T-matrix element. As a result, while converting the result of [12] to the notations of this paper, we have to multiply by a factor of $-i$. Second, [12] gave various results using the normalization constants $\mathcal{N}_{k}$ for the $(k, 1)$ instanton amplitudes, which will be related by the normalization constants $\mathbf{N}_{k}$ in our notation by $\mathcal{N}_{k}=i \mathbf{N}_{k}$. Therefore, in an expression in [12] containing $r$ factors of different $\mathcal{N}_{k}$ 's, there are $(r-1)$ hidden $i$ 's that will remain after we have stripped off the normalization factors of $\mathbf{N}$ from the T-matrix elements. Under complex conjugation this will pick up a factor of $(-1)^{r-1}$. The procedure discussed at the end of section 7.1 now tells us that in order to convert such an expression from the Lorentzian prescription to the unitary prescription, we need to anti-symmetrize the expression under $\omega_{i} \rightarrow-\omega_{i}$ for even $r$, and symmetrize the expression under $\omega_{i} \rightarrow-\omega_{i}$ for odd $r$. The expressions in [12] do not have explicit $i$ 's in the normalization factor, but if there were such factors then each factor of $i$ will shift the effective $r$ by 1 .

As an example we note that expression (7.7), after being converted to the notation of [12], would have two factors of $\mathcal{N}$. Therefore here $r=2$ and we have to antisymmetrize (7.7) under $\omega_{i} \rightarrow-\omega_{i}$ to get the result in the unitary prescription. This is what we had concluded in section 7.1. One point we should remember is that this antisymmetrization should not involve any factor coming from Liouville correlators. For the two point functions that we shall discuss, these factors remain unchanged under $\omega_{i} \rightarrow-\omega_{i}$, and so we do not need to be careful about this.

Let us now apply this to compute the leading order $e^{-3 / g_{s}}$ contribution to the 2-point function of closed strings, with the incoming string carrying energy $\tilde{\omega}_{1}=-\omega_{1}>0$ and the outgoing string carrying energy $\omega_{2}>0$. The contribution computed in [12] come from three sources. First the three instanton contribution to the S-matrix was given by:

$$
e^{-3 / g_{s}} 2 \pi \delta\left(\tilde{\omega}_{1}-\omega_{2}\right) \mathcal{N}_{1}^{3} \frac{64 \pi^{4}}{3} \sinh ^{2}\left(\pi \omega_{2}\right)\left(1+e^{-2 \pi \omega_{2}}+e^{-4 \pi \omega_{2}}\right),
$$

where $\mathcal{N}_{1}$ (called $\mathcal{N}$ in section 7.4) is the normalization factor associated with the one instanton contribution. Since there are 3 factors of $\mathcal{N}_{k}$, we need to symmetrize the expression under $\omega_{i} \rightarrow-\omega_{i}$ to get the result for unitary prescription. This gives:

$$
e^{-3 / g_{s}} 2 \pi \delta\left(\tilde{\omega}_{1}-\omega_{2}\right) \mathcal{N}_{1}^{3} \frac{64 \pi^{4}}{3} \sinh ^{2}\left(\pi \omega_{2}\right) \frac{1}{2}\left(2+e^{-2 \pi \omega_{2}}+e^{-4 \pi \omega_{2}}+e^{2 \pi \omega_{2}}+e^{4 \pi \omega_{2}}\right) .
$$

At the same order we have a two instanton contribution with one $(2,1)$ and one $(1,1)$ instanton. In the Lorentzian prescription this was given by:

$$
-e^{-3 / g_{s}} 2 \pi \delta\left(\tilde{\omega}_{1}-\omega_{2}\right) \mathcal{N}_{1} \mathcal{N}_{2} \frac{32 \pi^{2}}{3} \sinh ^{2}\left(\pi \omega_{2}\right)\left(e^{2 \pi \omega_{2}}+3+3 e^{-2 \pi \omega_{2}}+2 e^{-4 \pi \omega_{2}}\right),
$$

where $\mathcal{N}_{2}$ is the normalization factor associated with the $(2,1)$ instanton. Since we have two factors of $\mathcal{N}_{k}$, we need to antisymmetrize the expression under $\omega_{i} \rightarrow-\omega_{i}$ to get the corresponding expression in the unitary prescription. This gives:

$$
\begin{array}{r}
-e^{-3 / g_{s}} 2 \pi \delta\left(\tilde{\omega}_{1}-\omega_{2}\right) \mathcal{N}_{1} \mathcal{N}_{2} \frac{32 \pi^{2}}{3} \sinh ^{2}\left(\pi \omega_{2}\right) \frac{1}{2}\left(e^{2 \pi \omega_{2}}+3 e^{-2 \pi \omega_{2}}+2 e^{-4 \pi \omega_{2}}\right. \\
\left.-e^{-2 \pi \omega_{2}}-3 e^{2 \pi \omega_{2}}-2 e^{4 \pi \omega_{2}}\right) .
\end{array}
$$


The value of $\mathcal{N}_{2}$ in the unitary prescription has been given in (7.27). Finally the contribution from the single $(3,1)$ instanton takes the same form in the Lorentzian and the unitary prescription:

$$
e^{-3 / g_{s}} 2 \pi \delta\left(\tilde{\omega}_{1}-\omega_{2}\right) 4 \mathcal{N}_{3} \sinh ^{2}\left(3 \pi \omega_{2}\right) .
$$

The sum of (7.34), (7.36) and (7.37) should be compared with the expected answer from the matrix model [12]:

$$
\begin{array}{r}
-e^{-3 / g_{s}} 2 \pi \delta\left(\tilde{\omega}_{1}-\omega_{2}\right) \frac{1}{24 \pi^{2}} \sinh ^{2}\left(\pi \omega_{2}\right)\left[4+5 \cosh \left(2 \pi \omega_{2}\right)+3 \cosh \left(4 \pi \omega_{2}\right)\right. \\
\left.+2 \sinh \left(2 \pi \omega_{2}\right)+2 \sinh \left(4 \pi \omega_{2}\right)\right] .
\end{array}
$$

We find that the following choice of $\mathcal{N}_{3}$ satisfies the requirement:

$$
\mathcal{N}_{3}=-\frac{1}{96 \pi^{2}}
$$

Related observation has been made independently in [36]. In contrast in the Lorentzian prescription the required value of $\mathcal{N}_{3}$ was found [12] to be $-5 /\left(192 \pi^{2}\right)$.

\section{Contour prescription from Picard-Lefschetz theory}

In this section we shall use Picard-Lefschetz theory to argue that non-BPS D-instantons in type IIA string theory and instanton anti-instanton system in type IIB string theory give real contribution to the closed string effective action.

In Picard-Lefschetz theory (see [48-51, 63, 64] for review), we consider a finite dimensional integral of the form,

$$
I=\int_{\mathcal{C}}\left\{\prod_{i=1}^{N} d x_{i}\right\} e^{S\left(\left\{x_{i}\right\}\right)},
$$

where $S\left(\left\{x_{i}\right\}\right)$ is a function of $N$ variables $x_{1}, \cdots, x_{N}$, carrying an overall normalization factor $1 / g_{s}$ for some small parameter $g_{s}$, and $\mathcal{C}$ denotes some $N$ real dimensional subspace of the $N$ complex dimensional space of the $x_{i}$ 's. The integral is evaluated as follows. We first identify the critical points of $S$ in the complex $x_{i}$ space. Then for each critical point $\sigma$, we associate the Lefschetz thimble $\mathcal{J}_{\sigma}$, an $N$ real dimensional subspace of the $N$ complex dimensional space of the $x_{i}$ 's, by solving the gradient flow equations:

$$
\frac{d x_{i}}{d \tau}=\frac{\partial S^{*}}{\partial x_{i}^{*}}, \quad \frac{d x_{i}^{*}}{d \tau}=\frac{\partial S}{\partial x_{i}},
$$

where $\tau$ is a real parameter, * denotes complex conjugation and the flow lines approach the critical point $\sigma$ at $\tau \rightarrow \infty$. The collection of all such flow lines define $\mathcal{J}_{\sigma}$. We define

$$
I_{\sigma}=\int_{\mathcal{J}_{\sigma}}\left\{\prod_{i=1}^{N} d x_{i}\right\} e^{S\left(\left\{x_{i}\right\}\right)} .
$$

The original integration contour in (8.1) is then expressed as a weighted sum $\sum_{\sigma} n_{\sigma} \mathcal{J}_{\sigma}$ for appropriate choice of the multipliers $n_{\sigma}$ and the integral (8.1) is given by $\sum_{\sigma} n_{\sigma} I_{\sigma}$. 
An important point to keep in mind is that the integrals $I_{\sigma}$ do not change under small deformations of $\mathcal{J}_{\sigma}$ as long as the asymptotic boundaries are kept fixed (or kept within the range along which $S\left(\left\{x_{i}\right\}\right)$ approaches $-\infty$ sufficiently rapidly).

In the current context, the D-instanton systems represent the critical points in the configuration space of open and closed string fields, and the contribution to the amplitude from a given D-instanton system is expressed as the path integral over the open and closed string fields along the thimble corresponding to that particular instanton, obtained by solving (8.2). However, since our interest is in computing the closed string effective action by integrating out the open string modes, we can focus on the integration over the open string modes only. Furthermore, the massive open string modes can be integrated out first by standard procedure, leading to an effective action of the massless and tachyonic open string modes and closed strings. We can also drop the integration over the collective coordinates describing the center of degrees of freedom of the full system of D-instantons, since the dependence on these modes is trivial. Therefore we focus on integration over the rest of the massless modes and tachyonic open string modes only. These are finite in number. Of course the effective action involving these modes and the closed string modes is not known, and is likely to be very complicated. However, since the result of integration depends only on the topological class of the integration contour and not on the details, we expect that the essential features of the integration contours can be captured by working with some prototype actions that capture the essential properties of these modes. Finally, since we treat the closed strings states perturbatively, expanding the action in powers of these fields, the integration contours over the open string modes can be analyzed by examining the part of the action obtained by setting the closed string modes to zero, and then using the same integration contour even in the presence of the closed strings. For this reason, we shall drop the closed string fields during the analysis of the integration contour, and consider the integration over the tachyonic and massless open string modes, weighted by the exponential of the effective action of these modes. Our goal will be to express the desired integration contour on the open string modes as weighted union of the thimbles associated with different critical points, thereby resolving some of the ambiguities encountered earlier in determining the contribution from different instantons.

\subsection{Non-BPS D-instanton in type IIA string theory}

The non-BPS D-instanton in type IIA string theory has a real tachyonic mode. The tachyon potential is symmetric under $T \rightarrow-T$, with a maximum at $T=0$ representing the $\mathrm{D}$ instanton and a pair of minima at some values representing the perturbative vacuum [62]. Furthermore, the two minima are expected to describe the same perturbative vacuum. For this reason it is more appropriate to regard the tachyon as an angular variable. We shall denote by $\theta$ the appropriately normalized angular variable and use the following form the action:

$$
S(\theta)=-\frac{1}{\sqrt{2} g_{s}}(1+\cos \theta) .
$$

The point $\theta=0$, with action $-\sqrt{2} / g_{s}$, describes the D-instanton configuration and $\theta=\pi$, with action 0 , denotes the vacuum without $\mathrm{D}$-instanton. The natural choice for the original 


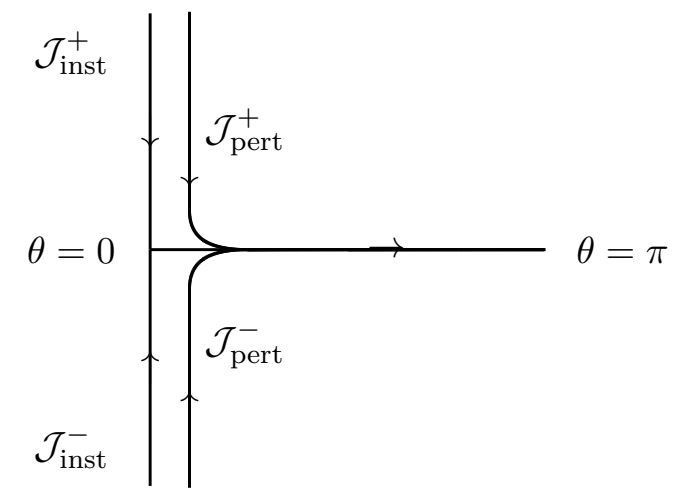

Figure 15. The steepest descent contours for the action (8.4).

integration contour is along the real $\theta$ axis. We shall restrict the integration range to $0 \leq \theta \leq \pi$. We note however that the qualitative features of our result will not depend on the details of the potential - in particular we shall get similar results also for the standard double well potential.

For real positive $g_{s}$, the steepest descent contour for the saddle point at $\theta=\pi$, representing the perturbative vacuum, runs along the real axis from 0 to $\pi$. Let us call this $\mathcal{J}_{\text {pert }}$. On the other hand, there is a pair of steepest descent contours associated with the saddle point at 0 , representing the instanton, running along the imaginary axis from $\pm i \infty$ to 0 . We shall denote these by $\mathcal{J}_{\text {inst }}^{ \pm}$. Therefore the integration cycle contains only the steepest descent contour associated with the perturbative vacuum. In our convention, this can be summarized by saying that the normalization constant $\mathbf{N}$, associated with the instanton, vanishes.

If instead of taking $g_{s}$ to be real, we take this to have a small imaginary part, then the steepest descent contours have more intricate structure [49]. The steepest descent contours associated with the instanton are only slightly deformed from their original form, but only one of them stays inside the range $0 \leq \operatorname{Re}(\theta) \leq \pi$. Whether it is $\mathcal{J}_{\text {inst }}^{+}$or $\mathcal{J}_{\text {inst }}^{-}$depends on the sign of the imaginary part of $g_{s}$. The steepest descent contour associated with the perturbative vacuum now starts at $\pm i \infty$, runs towards zero almost along the imaginary axis and then runs almost along the real axis till $\theta=\pi$. Whether it begins at $i \infty$ or $-i \infty$ depends on the sign of the imaginary part of $g_{s}$. This has been shown by the contours $\mathcal{J}_{\text {pert }}^{ \pm}$in figure 15 . Therefore in this case the integration cycle will be given by $\mathcal{J}_{\text {pert }}^{ \pm}-\mathcal{J}_{\text {inst }}^{ \pm}$, and whether we use + or - sign depends on the sign of the imaginary part of $g_{s}$. This shows that in this case there is a contribution from the steepest descent contour associated with the instanton. However now since the steepest descent contour $\mathcal{J}_{\text {pert }}^{ \pm}$is deformed into the complex plane, its contribution is not real, and the imaginary part cancels with the one coming from $\mathcal{J}_{\text {inst }}^{ \pm}$in the limit of real $g_{s}$. We can avoid this by taking the average between the limits from the $\operatorname{Im}\left(g_{s}\right)>0$ and $\operatorname{Im}\left(g_{s}\right)<0$ sides. In this case the imaginary contributions to $\mathcal{J}_{\text {pert }}^{ \pm}$cancel, but the contributions $\mathcal{J}_{\text {inst }}^{ \pm}$from the steepest descent contours associated with the instanton also cancel. Therefore we are again led to the result $\mathbf{N}=0$.

Note that since the full result is continuous across the real axis, the limits from the $\operatorname{Im}\left(g_{s}\right)>0$ and $\operatorname{Im}\left(g_{s}\right)<0$ sides give the same result. However for this we need to include 
the contributions from the steepest descent contours for the perturbative vacuum and the instanton. Only by taking the average, or by working strictly with real $g_{s}$, we get vanishing contribution from the instanton and real contribution from the perturbative vacuum.

We must also remind the reader that the results described above depend crucially on the original choice of the integration contour. For example, if instead of taking the integration over the tachyon to run along the real axis we had taken it to run from $-i \infty$ to $i \infty$, then it can be expressed as $\mathcal{J}_{\text {inst }}^{-}-\mathcal{J}_{\text {inst }}^{+}$and we shall get imaginary contribution to the integral.

\subsection{Instanton anti-instanton system in type IIB string theory}

We now turn to the system containing a D-instanton anti-D-instanton pair in type IIB string theory. In this case the relevant degrees of freedom include the mode describing the relative location between the D-instanton and the anti-D-instanton, and the complex scalar mode $T$ that becomes tachyonic when the separation is less than a critical value. At zero separation, the tachyon potential has a maximum at $T=0$ representing the instanton anti-instanton system, and a circle of minima at a non-zero value of $|T|$ representing the perturbative vacuum without any instantons [62]. There is strong reason to believe, however, that the circle of minima, differing by the choice of phase of $T$, describes the same vacuum. For this reason, it will be more appropriate to represent the space of tachyons as a sphere labelled by polar angles $(\theta, \phi)$, with $\theta=0$ denoting the maximum of the potential and $\theta=\pi$ denoting the minimum of the potential. On the other hand the relative location between the D-instantons is described by 10 real coordinates since type IIB D-instanton has 10 transverse space-time coordinates. However only the distance variable $\chi$ will be of importance in our analysis, since using the spherical symmetry in ten dimensions, the integration over the angular variable can be factored out of the integral. Therefore we consider an integral over $\theta, \phi, \chi$ and model the action by,

$$
S(\theta, \phi, \chi)=-\frac{1}{g_{s}}\left[(1+\cos \theta)+\chi^{2}(1-\cos \theta)\right] .
$$

Here $\theta=0$ represents the instanton anti-instanton configuration, separated by distance $\chi$, with action $-2 / g_{s}$. On the other hand, $\theta=\pi, \chi=0$ represents the vacuum without $\mathrm{D}$ instanton with vanishing action. Note that we have appropriately rescaled $\chi$ to avoid factors of $2 \pi$. For definiteness, the integration measure over $(\theta, \phi)$ will be taken to be $\sin \theta d \theta d \phi$ although full spherical symmetry in $(\theta, \phi)$ space is not guaranteed, and the integration measure over $\chi$ will be taken as $\chi^{9} d \chi$. Natural choice of the integration contour $\mathcal{C}$ is the real $\theta-\phi-\chi$ plane and the integral is finite and real, except for a possible divergence from the large $\chi$, small $\theta$ region. However, as we have discussed below (7.14), in the construction of the closed string effective action this integral is cut off at large $\chi$, and there is no divergence.

We shall now describe the decomposition of the integration contour in terms of the Lefschetz thimbles around the saddle points. Since the integrand does not depend on $\phi$, we can carry out the $\phi$ integral, picking a factor of $2 \pi$. Eventually this will be canceled when we divide the result by the volume of the rigid $U(1)$ gauge groups living on the (anti-) D-instanton. Therefore we shall not discuss this any further. Furthermore, we shall 


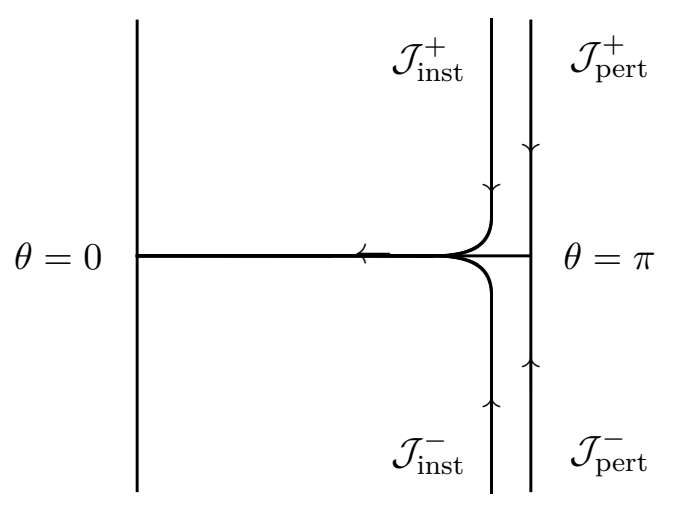

Figure 16. The steepest descent contours for the action (8.5) in the complex $\theta$ plane for $x^{2}>1$.

not use the method of steepest descent to carry out the integration over $\chi$ - instead we shall envisage carrying out the integral over $\chi$ at the end explicitly after carrying out the integration over $\theta$ using the steepest descent contours for fixed $\chi$. This reduces the problem to the one discussed in section 8.1, with $g_{s} /\left(1-\chi^{2}\right)$ representing the effective coupling. Since this blows up at $\chi=1$, the method of steepest descent breaks down, although the full integral remains finite. For this reason we shall evaluate the integral by deforming the $\chi$ integration contour into the complex plane around $\chi=1$. Since the full integral is non-singular at $\chi=1$, the result does not depend on how we deform the contour. However following our discussion in section 8.1, we see that if we want to keep the contribution from the steepest descent contour associated with the perturbative vacuum real, we need to average over the contours above and below 1 in the complex $\chi$ plane, and this is the route we shall follow.

For the steepest descent contours associated with the saddle point at $\theta=0$, the integration over $\theta$ now produces the factor proportional to $e^{-2 / g_{s}}\left(\chi^{2}-1\right)^{-1}$ that appeared e.g. in (5.14), and averaging over the contours above and below the pole at 1 , we get the unitary prescription for the contribution from this pair of saddle points. On the other hand, integration over $\theta$ for the steepest descent contour associated with the saddle point at $\theta=\pi$ produces the factor proportional to $-e^{-2 \chi^{2} / g_{s}}\left(\chi^{2}-1\right)^{-1}$. Taking the average over the contours $\mathcal{J}_{\text {pert }}^{+}$and $\mathcal{J}_{\text {pert }}^{-}$we get real contribution from the perturbative vacuum as well.

If we label the contour near $\chi=1$ as $\chi=1+\epsilon e^{i \alpha}$ and vary $\alpha$ from $\pi$ to 0 or $-\pi$ to 0 depending on whether the contour passes above or below $\chi=1$, the role of the two saddle points get exchanged as $\alpha$ crosses $\pm \pi / 2$. For $\operatorname{Re}(\chi)>1$, the saddle point at $\theta=0$ becomes the dominant saddle point and the steepest descent contours in the complex $\theta$ plane get deformed as shown in figure 16 .

\subsection{Instanton in two dimensional bosonic string theory}

An instanton in two dimensional bosonic string theory has a real tachyonic mode $T$ with potential that has a maximum at $T=0$ representing the D-instanton and a local minimum at a positive value of $T$ representing the perturbative vacuum. The potential approaches 


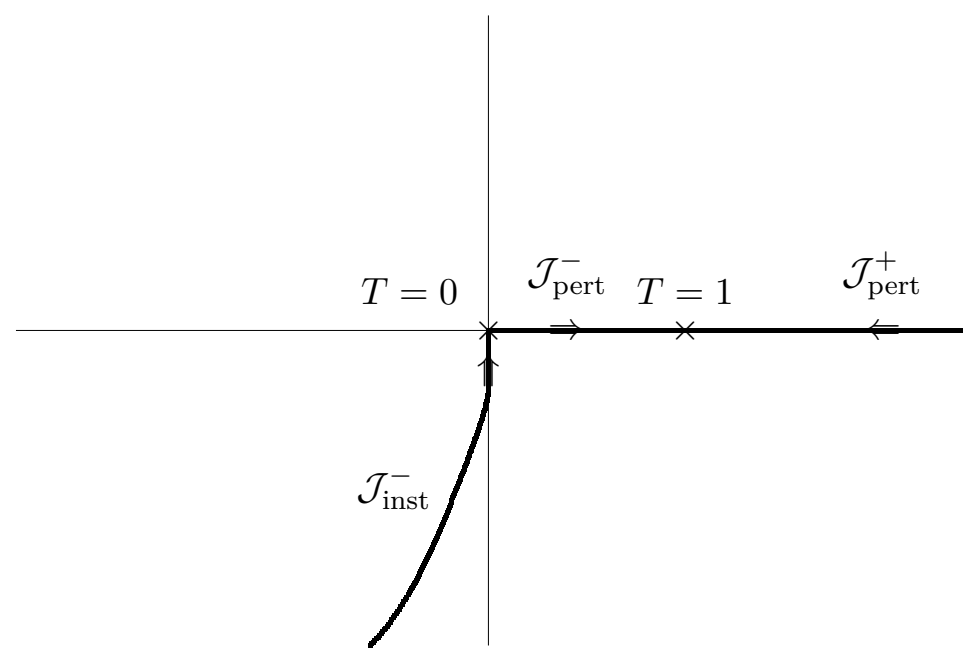

Figure 17. The steepest descent contours for the action (8.6) in the complex $T$ plane.

$\pm \infty$ as $T \rightarrow \pm \infty$. We can model this by choosing:

$$
S(T)=-\frac{1}{g_{s}}\left(1-3 T^{2}+2 T^{3}\right)
$$

In this case a real integration contour over $T$ does not give a well defined result, since $S(T)$ approaches $\infty$ as $T \rightarrow-\infty$. Instead, the allowed asymptotic regions in which the contour must lie consists of three cones, each of opening angle $\pi / 3$, around the lines $\operatorname{Arg}[T]=0$ and $\operatorname{Arg}[T]= \pm 2 \pi i / 3$ (see e.g. the discussion on Airy function in [48, 63]). Therefore we have to choose a contour in the complex $T$ plane. This makes the result complex. For physical reasons, we would like the integral, representing the contribution to the closed string effective action, to have positive imaginary part so that it represents a dissipative system. Furthermore, the contour should pass through the perturbative vacuum at $T=1$. This can be achieved by choosing the integration contour so that asymptotically it lies within the cones around $\operatorname{Arg}[T]=0$ and $\operatorname{Arg}[T]=-2 \pi i / 3$. This has been shown in figure 17 by the thick line.

The saddle point at $T=0$ with action $-1 / g_{s}$ represents the unstable D-instanton, while the saddle point at $T=1$ with vanishing action represents the vacuum without any D-instanton. The steepest descent contours for $T=1$ run along the real axis from 0 to 1 and $\infty$ to 1 . We shall denote these by $\mathcal{J}_{\text {pert }}^{-}$and $\mathcal{J}_{\text {pert }}^{+}$respectively. The steepest descent contours for the saddle point at $T=0$ run from $e^{ \pm 2 \pi i / 3} \infty$ to 0 . We shall denote these by $\mathcal{J}_{\text {inst }}^{ \pm}$respectively. Then we have, from figure 17 ,

$$
\mathcal{C}=\mathcal{J}_{\text {inst }}^{-}+\mathcal{J}_{\text {pert }}^{-}-\mathcal{J}_{\text {pert }}^{+}
$$

The first terms gives complex contribution while the last two terms give real contribution. This provides a qualitative understanding of why in this case the D-instanton contribution to the closed string effective action may be complex. 


\subsection{Final remarks}

Although the analysis described above gives a qualitative understanding of the ambiguities involved in computing D-instanton contribution to the closed string effective action, we need to keep in mind that the full problem is more involved and new features may appear there. For example, from the point of view of open string theory, the saddle point representing the perturbative vacuum is the dominant saddle, and its contribution should dominate the closed string effective action. However, it is known that the boundary state of the D-instanton vanishes at that saddle [72]. Therefore we would expect that all closed string amplitudes on Riemann surfaces with boundaries ending on the D-instanton, on which open strings have condensed to the tachyon vacuum, will actually vanish. This suggests that the perturbative contribution to the closed string effective action from the dominant saddle point actually vanishes (unless we include the purely closed string contribution $S_{0}$ as part of the contribution from this saddle point). Nevertheless we would expect a nonperturbative contribution to survive at least for complex $g_{s}$, since in the $\operatorname{Im}\left(g_{s}\right) \rightarrow 0^{ \pm}$limit, these contributions are supposed to cancel the imaginary parts of the contribution from the Lefschetz thimble associated with the D-instanton. It will certainly be important to understand this better.

The general lesson that is likely to survive in the full theory is that when the tachyon potential is bounded from below, as in superstring theory, we can choose real integration contour over the open string modes on the D-instanton, and therefore integration over these open string modes will give real contribution to the closed string effective action.

\section{Conclusions}

The main results of this paper may be summarized as follows:

1. We have described a systematic procedure for constructing a novel gauge invariant effective action obtained by integrating out the open string modes on the D-instanton. This differs from the usual method of integrating out a subset of the degrees of freedom, in that we need to sum over connected and disconnected diagrams in defining the effective action, and furthermore we have an unknown normalization constant $\mathbf{N} e^{-C / g_{s}}$ appearing in the expression for the effective action. Nevertheless, the effective action constructed this way satisfies the BV master equation.

2. We have shown that cutting rules and unitarity of the amplitudes follow as long as the effective action constructed this way is real. For multiple D-instantons and multiple anti-D-instantons in type IIB string theory, the reality condition holds and their contribution to the amplitude is unitary.

3. For D-instanton systems with tachyonic modes, e.g. instanton anti-instanton system in type IIB string theory and the non-BPS D-instanton in type IIA string theory, the reality condition could fail due to two effects: the overall normalization $\mathbf{N}$ could be complex and the contour prescription for integrating over the relative positions of the instantons and anti-instantons may lead to complex effective action. However, 
using insights from Picard-Lefschetz theory we have argued that when the tachyon potential is bounded from below, as in the case of critical superstring theories, the effective action is actually real.

4. Non-unitarity of two dimensional string theory identified in [11, 12] can be traced to both the sources mentioned above. In particular the overall normalization $\mathbf{N}$, found in [11] by comparison with matrix model results, is purely imaginary. Furthermore, in the multi-instanton sector, [12] used the 'Lorentzian' prescription to integrate over the relative locations of the instantons, leading to complex contribution to the effective action. However, if we do not attempt comparison with the matrix model results, we can in principle recover unitary D-instanton amplitudes in two dimensional bosonic string theory by taking $\mathbf{N}$ to be real and using unitary contour prescription for integrating over the relative locations of the instantons.

\section{Acknowledgments}

I wish to thank Bruno Balthazar, Victor Rodriguez and Xi Yin for many useful discussions and for informing me of many of their unpublished results. I also wish to thank Michael Green, Michael Gutperle and Barton Zwiebach for useful discussions. This work was supported in part by the J.C. Bose fellowship of the Department of Science and Technology, India and the Infosys chair professorship.

Open Access. This article is distributed under the terms of the Creative Commons Attribution License (CC-BY 4.0), which permits any use, distribution and reproduction in any medium, provided the original author(s) and source are credited.

\section{References}

[1] R.E. Cutkosky, Singularities and discontinuities of Feynman amplitudes, J. Math. Phys. 1 (1960) 429 [INSPIRE].

[2] M. Fowler, Introduction to Momentum Space Integration Techniques in Perturbation Theory, J. Math. Phys. 3 (1962) 936.

[3] M.J.G. Veltman, Unitarity and causality in a renormalizable field theory with unstable particles, Physica 29 (1963) 186 [INSPIRE].

[4] G. 't Hooft and M.J.G. Veltman, DIAGRAMMAR, NATO Sci. Ser. B 4 (1974) 177 [INSPIRE].

[5] S. Bloch and D. Kreimer, Cutkosky Rules and Outer Space, arXiv:1512.01705 [INSPIRE].

[6] R. Pius and A. Sen, Cutkosky rules for superstring field theory, JHEP 10 (2016) 024 [Erratum ibid. 09 (2018) 122] [arXiv: 1604.01783] [INSPIRE].

[7] J. Polchinski, Combinatorics of boundaries in string theory, Phys. Rev. D 50 (1994) R6041 [hep-th/9407031] [INSPIRE].

[8] M.B. Green and M. Gutperle, Effects of D instantons, Nucl. Phys. B 498 (1997) 195 [hep-th/9701093] [INSPIRE].

[9] A. Sen, Rolling tachyon, JHEP 04 (2002) 048 [hep-th/0203211] [INSPIRE]. 
[10] A. Sen, Tachyon matter, JHEP 07 (2002) 065 [hep-th/0203265] [INSPIRE].

[11] B. Balthazar, V.A. Rodriguez and X. Yin, ZZ Instantons and the Non-Perturbative Dual of $c=1$ String Theory, arXiv:1907.07688 [InSPIRE].

[12] B. Balthazar, V.A. Rodriguez and X. Yin, Multi-Instanton Calculus in $c=1$ String Theory, arXiv: 1912.07170 [INSPIRE].

[13] N.D. Lambert, H. Liu and J.M. Maldacena, Closed strings from decaying D-branes, JHEP 03 (2007) 014 [hep-th/0303139] [INSPIRE].

[14] B. Sundborg, Selfenergies of Massive Strings, Nucl. Phys. B 319 (1989) 415 [InSPIRE].

[15] K. Amano and A. Tsuchiya, Mass Splittings and the Finiteness Problem of Mass Shifts in the Type II Superstring at One Loop, Phys. Rev. D 39 (1989) 565 [inSPIRE].

[16] N. Marcus, Unitarity and Regularized Divergences in String Amplitudes, Phys. Lett. B 219 (1989) 265 [INSPIRE].

[17] B. Sundborg, Infinite Mass Shifts Of Open Superstrings As A Possible Signal Of Confinement, Nucl. Phys. B 338 (1990) 101 [INSPIRE].

[18] E. D'Hoker and D.H. Phong, Momentum analyticity and finiteness of the one loop superstring amplitude, Phys. Rev. Lett. 70 (1993) 3692 [hep-th/9302003] [INSPIRE].

[19] E. D'Hoker and D.H. Phong, Dispersion relations in string theory, Theor. Math. Phys. 98 (1994) 306 [hep-th/9404128] [INSPIRE].

[20] E. D'Hoker and D.H. Phong, The box graph in superstring theory, Nucl. Phys. B 440 (1995) 24 [hep-th/9410152] [INSPIRE].

[21] A. Berera, Unitary string amplitudes, Nucl. Phys. B 411 (1994) 157 [inSPIRE].

[22] E. Witten, The Feynman iє in String Theory, JHEP 04 (2015) 055 [arXiv:1307.5124] [INSPIRE].

[23] H. Kajiura, Homotopy algebra morphism and geometry of classical string field theory, Nucl. Phys. B 630 (2002) 361 [hep-th/0112228] [INSPIRE].

[24] H. Kajiura, Noncommutative homotopy algebras associated with open strings, Rev. Math. Phys. 19 (2007) 1 [math/0306332] [INSPIRE].

[25] A. Sen, Wilsonian Effective Action of Superstring Theory, JHEP 01 (2017) 108 [arXiv: 1609.00459] [INSPIRE].

[26] H. Erbin, C. Maccaferri, M. Schnabl and J. Vošmera, Classical algebraic structures in string theory effective actions, JHEP 11 (2020) 123 [arXiv: 2006.16270] [INSPIRE].

[27] A. Abouelsaood, C.G. Callan Jr., C.R. Nappi and S.A. Yost, Open Strings in Background Gauge Fields, Nucl. Phys. B 280 (1987) 599 [InSPIRE].

[28] C.G. Callan Jr., C. Lovelace, C.R. Nappi and S.A. Yost, Adding Holes and Crosscaps to the Superstring, Nucl. Phys. B 293 (1987) 83 [InSPIRE].

[29] J. Polchinski and Y. Cai, Consistency of Open Superstring Theories, Nucl. Phys. B 296 (1988) 91 [INSPIRE].

[30] N. Ishibashi, The Boundary and Crosscap States in Conformal Field Theories, Mod. Phys. Lett. A 4 (1989) 251 [INSPIRE].

[31] M.B. Green and M. Gutperle, Light cone supersymmetry and D-branes, Nucl. Phys. B 476 (1996) 484 [hep-th/9604091] [inSPIRE]. 
[32] P. Di Vecchia, M. Frau, I. Pesando, S. Sciuto, A. Lerda and R. Russo, Classical p-branes from boundary state, Nucl. Phys. B 507 (1997) 259 [hep-th/9707068] [INSPIRE].

[33] P. Di Vecchia and A. Liccardo, D-branes in string theory. 2, in YITP Workshop on Developments in Superstring and M-theory, (1999) [hep-th/9912275] [INSPIRE].

[34] M.B. Green, Point-like states for type 2b superstrings, Phys. Lett. B 329 (1994) 435 [hep-th/9403040] [INSPIRE].

[35] V. Rodriguez, talk at the Workshop on Matrix Models and String Theory, https://www.youtube.com/watch?v=0Nj-T8lgB4E.

[36] B. Balthazar, V.A. Rodriguez and X. Yin, private communications.

[37] A.B. Zamolodchikov and A.B. Zamolodchikov, Liouville field theory on a pseudosphere, hep-th/0101152 [INSPIRE].

[38] B. Zwiebach, Oriented open-closed string theory revisited, Annals Phys. 267 (1998) 193 [hep-th/9705241] [INSPIRE].

[39] S. Faroogh Moosavian, A. Sen and M. Verma, Superstring Field Theory with Open and Closed Strings, JHEP 01 (2020) 183 [arXiv:1907.10632] [INSPIRE].

[40] S.R. Das and A. Jevicki, String Field Theory and Physical Interpretation of D $=1$ Strings, Mod. Phys. Lett. A 5 (1990) 1639 [inSPIRE].

[41] A.M. Sengupta and S.R. Wadia, Excitations and interactions in $d=1$ string theory, Int. J. Mod. Phys. A 6 (1991) 1961 [InSPIRE].

[42] D.J. Gross and I.R. Klebanov, Fermionic string field theory of $c=1$ two-dimensional quantum gravity, Nucl. Phys. B 352 (1991) 671 [InSPIRE].

[43] I.R. Klebanov, String theory in two-dimensions, in Spring School on String Theory and Quantum Gravity (to be followed by Workshop), (1991) [hep-th/9108019] [INSPIRE].

[44] G.W. Moore, M.R. Plesser and S. Ramgoolam, Exact $S$ matrix for 2-D string theory, Nucl. Phys. B 377 (1992) 143 [hep-th/9111035] [INSPIRE].

[45] T. Takayanagi and N. Toumbas, A matrix model dual of type $O B$ string theory in two-dimensions, JHEP 07 (2003) 064 [hep-th/0307083] [INSPIRE].

[46] M.R. Douglas, I.R. Klebanov, D. Kutasov, J.M. Maldacena, E.J. Martinec and N. Seiberg, A new hat for the c=1 matrix model, in From Fields to Strings: Circumnavigating Theoretical Physics: A Conference in Tribute to Ian Kogan, (2003) [hep-th/0307195] [INSPIRE].

[47] B. Balthazar, V.A. Rodriguez and X. Yin, The $c=1$ string theory S-matrix revisited, JHEP 04 (2019) 145 [arXiv: 1705.07151] [INSPIRE].

[48] M. Mariño, Lectures on non-perturbative effects in large $N$ gauge theories, matrix models and strings, Fortsch. Phys. 62 (2014) 455 [arXiv: 1206.6272] [INSPIRE].

[49] G.V. Dunne and M. Ünsal, What is QFT? Resurgent trans-series, Lefschetz thimbles, and new exact saddles, PoS LATTICE2015 (2016) 010 [arXiv:1511.05977] [INSPIRE].

[50] G.V. Dunne and M. Ünsal, New Nonperturbative Methods in Quantum Field Theory: From Large-N Orbifold Equivalence to Bions and Resurgence, Ann. Rev. Nucl. Part. Sci. 66 (2016) 245 [arXiv: 1601.03414] [INSPIRE].

[51] I. Aniceto, G. Basar and R. Schiappa, A Primer on Resurgent Transseries and Their Asymptotics, Phys. Rept. 809 (2019) 1 [arXiv: 1802.10441] [INSPIRE]. 
[52] A. Sen, Unitarity of Superstring Field Theory, JHEP 12 (2016) 115 [arXiv:1607.08244] [INSPIRE].

[53] A. Sen, D-instanton Perturbation Theory, JHEP 08 (2020) 075 [arXiv:2002.04043] [INSPIRE].

[54] A. Sen, Reality of Superstring Field Theory Action, JHEP 11 (2016) 014 [arXiv: 1606.03455] [INSPIRE].

[55] H. Sonoda and B. Zwiebach, Closed String Field Theory Loops With Symmetric Factorizable Quadratic Differentials, Nucl. Phys. B 331 (1990) 592 [InSPIRE].

[56] B. Zwiebach, Closed string field theory: Quantum action and the B-V master equation, Nucl. Phys. B 390 (1993) 33 [hep-th/9206084] [INSPIRE].

[57] M.R. Gaberdiel and B. Zwiebach, Tensor constructions of open string theories. 1: Foundations, Nucl. Phys. B 505 (1997) 569 [hep-th/9705038] [INSPIRE].

[58] I.A. Batalin and G.A. Vilkovisky, Gauge Algebra and Quantization, Phys. Lett. B 102 (1981) 27 [INSPIRE].

[59] I.A. Batalin and G.A. Vilkovisky, Quantization of Gauge Theories with Linearly Dependent Generators, Phys. Rev. D 28 (1983) 2567 [Erratum ibid. 30 (1984) 508] [INSPIRE].

[60] A. Sen, D-instantons, String Field Theory and Two Dimensional String Theory, to appear.

[61] T. Lada and J. Stasheff, Introduction to SH Lie algebras for physicists, Int. J. Theor. Phys. 32 (1993) 1087 [hep-th/9209099] [INSPIRE].

[62] A. Sen, Tachyon dynamics in open string theory, Int. J. Mod. Phys. A 20 (2005) 5513 [hep-th/0410103] [INSPIRE].

[63] E. Witten, Analytic Continuation Of Chern-Simons Theory, AMS/IP Stud. Adv. Math. 50 (2011) 347 [arXiv: 1001.2933] [INSPIRE].

[64] E. Witten, A New Look At The Path Integral Of Quantum Mechanics, arXiv:1009.6032 [INSPIRE].

[65] M.B. Green and P. Vanhove, Duality and higher derivative terms in M-theory, JHEP 01 (2006) 093 [hep-th/0510027] [INSPIRE].

[66] M.B. Green, S.D. Miller and P. Vanhove, $\mathrm{SL}(2, \mathbb{Z})$-invariance and D-instanton contributions to the $D^{6} R^{4}$ interaction, Commun. Num. Theor. Phys. 09 (2015) 307 [arXiv:1404.2192] [INSPIRE].

[67] S.M. Chester, M.B. Green, S.S. Pufu, Y. Wang and C. Wen, New modular invariants in $\mathcal{N}=4$ Super-Yang-Mills theory, JHEP 04 (2021) 212 [arXiv:2008.02713] [INSPIRE].

[68] A. Sen, Divergent to Complex Amplitudes in Two Dimensional String Theory, JHEP 02 (2021) 086 [arXiv: 2003.12076] [INSPIRE].

[69] H. Dorn and H.J. Otto, Two and three point functions in Liouville theory, Nucl. Phys. B 429 (1994) 375 [hep-th/9403141] [INSPIRE].

[70] A.B. Zamolodchikov and A.B. Zamolodchikov, Structure constants and conformal bootstrap in Liouville field theory, Nucl. Phys. B 477 (1996) 577 [hep-th/9506136] [InSPIRE].

[71] A. Sen, One Loop Mass Renormalization of Unstable Particles in Superstring Theory, JHEP 11 (2016) 050 [arXiv: 1607.06500] [INSPIRE].

[72] M. Kiermaier, Y. Okawa and B. Zwiebach, The boundary state from open string fields, arXiv:0810.1737 [INSPIRE]. 